TRANSACTIONS OF THE

AMERICAN MATHEMATICAL SOCIETY

Volume 359, Number 10, October 2007, Pages 5057-5089

S 0002-9947(07)04219-5

Article electronically published on April 16, 2007

\title{
THE CLASS NUMBER ONE PROBLEM FOR THE NORMAL CM-FIELDS OF DEGREE 32
}

\author{
SUN-MI PARK, HEE-SUN YANG, AND SOUN-HI KWON
}

\begin{abstract}
We prove that there are exactly six normal CM-fields of degree 32 with class number one. Five of them are composita of two normal CM-fields of degree 16 with the same maximal totally real octic field.
\end{abstract}

\section{INTRODUCTION}

All the normal CM-fields of degree less than 32 with class number one are known. In this paper we will prove the following:

Theorem 1. (1) There is one and only one normal CM-field of degree 32 with class number one which is not a compositum of two normal CM-subfields of degree 16 with the same maximal real subfield: the narrow Hilbert class field of $\mathbb{Q}(\sqrt{5 \cdot 29}, \sqrt{2})$.

(2) There are exactly five normal CM-fields of degree 32 with relative class number one which are composita of two normal CM-fields of degree 16 with the same maximal real subfield : $\mathbb{Q}(\sqrt{2+\sqrt{2}}, \sqrt{3+\sqrt{3}}, \sqrt{-1})$, $\mathbb{Q}(\sqrt{2}, \sqrt{17+4 \sqrt{17}}, \sqrt{-(5+\sqrt{17}) / 2}, \sqrt{-(17+3 \sqrt{17}) / 2}, \alpha)$ with $\alpha^{8}+$ $17 \alpha^{6}+85 \alpha^{4}+136 \alpha^{2}+68=0, \mathbb{Q}(\theta, \sqrt{2}, \sqrt{-1}), \mathbb{Q}(\theta, \sqrt{3}, \sqrt{-1})$, and $\mathbb{Q}(\theta, \sqrt{7}, \sqrt{-1})$ with $\theta^{8}+10 \theta^{6}+25 \theta^{4}+20 \theta^{2}+5=0$. Moreover, those five fields have class number one.

The structure of this paper is as follows. In Section 2 we review well-known results on relative class numbers of CM-fields which will be used later in this paper. According to [LOO, Lemma 2 (ii)] a totally imaginary normal number field is a CMfield if and only if the complex conjugation lies in the center of its Galois group. A normal CM-field of degree 32 is a compositum of two normal CM-fields of degree 16 with the same maximal totally real octic field if and only if the center of its Galois group is not cyclic, and there are 44 non-abelian groups of order 32: 29 out of them have non-cyclic centers. We will study all of these 44 groups. In Section 4 we focus on such composita. Section 3 is devoted to the normal CM-fields of degree 32 that are not such composita of two normal CM-fields of degree 16 with the same maximal totally real octic field.

Received by the editors May 6, 2004 and, in revised form, September 30, 2005.

2000 Mathematics Subject Classification. Primary 11R29; Secondary 11R21.

(C)2007 American Mathematical Society

Reverts to public domain 28 years from publication 


\section{Preliminaries}

Throughout this paper we use the following notation. For a number field $F$, let $O_{F}, d_{F}, \zeta_{F}, h_{F}, \operatorname{Hil}(F), \operatorname{Hil}_{n a r}(F)$, and $w_{F} \geq 2$ be the ring of algebraic integers, the absolute value of discriminant, the Dedekind zeta function, the class number, the Hilbert class field, the narrow Hilbert class field of $F$, and the number of roots of unity in $F$, respectively. Let $\kappa_{F}$ denote the residue of $\zeta_{F}(s)$ at $s=1$. When $F$ is an abelian number field, let $G(F)$ denote its genus field. For a finite abelian extension $K$ of a number field $k$ let $\mathcal{F}(K / k)$ be the finite part of the conductor of the extension $K / k$. When $F$ is a totally real number field of degree $n$ over $\mathbb{Q}$, we denote by $\infty$ the product of its $n$ real infinite primes. When $F$ is a CM-field, we will denote by $F^{+}, h_{F}^{-}$, and $Q_{F} \in\{1,2\}$, the maximal totally real subfield, the relative class number, and the Hasse unit index of $F$, respectively. For a Galois extension $K / k$ we denote by $G(K / k)$ its Galois group. For a subgroup $H$ of $G(K / k)$, the fixed field of $H$ is denoted by $H^{\prime}$. Let $D_{n}$ be the dihedral group of order $n, C_{n}$ the cyclic group of order $n$, and $Q_{n}$ the dicyclic group of order $n$. ( $Q_{8}$ is usually said to be the quaternion group of order 8.) For a group $H$ let $C(H)$ be the center of $H, D(H)$ the commutator subgroup of $H$, and let $H^{a b}=H / D(H)$.

Theorem 2. (1) (LO2, Proposition 2]) If a CM-field $N=N_{1} N_{2}$ is a compositum of two CM-fields $N_{1}$ and $N_{2}$ with the same maximal totally real subfield $N_{1}^{+}=N_{2}^{+}$, then

$$
h_{N}^{-}=\frac{Q_{N}}{Q_{N_{1}} Q_{N_{2}}} \frac{w_{N}}{w_{N_{1}} w_{N_{2}}} h_{N_{1}}^{-} h_{N_{2}}^{-}
$$

and $h_{N_{1}}^{-} h_{N_{2}}^{-}$divides $4 h_{N}^{-}$. In particular, if $N_{1}$ and $N_{2}$ are isomorphic, then

$$
h_{N}^{-}=\frac{Q_{N}}{2}\left(\frac{h_{N_{1}}^{-}}{Q_{N_{1}}}\right)^{2} .
$$

(2) ([k, Theorem 1] and [Lem1, Proposition 1 (d)]) Let $M_{1} \subseteq M_{2}$ be two $C M$-fields. Then $h_{M_{1}}^{-} \mid 4 h_{M_{2}}^{-}$, and $Q_{M_{1}} w_{M_{1}}$ divides $Q_{M_{2}} w_{M_{2}}$.

(3) ([Lou2, Proposition 6]) Let $M$ be a $C M$-field and the number of ramified prime ideals in $M / M^{+}$. Then $2^{t-1}$ divides $h_{M}^{-}$and $2^{t}$ divides $h_{M}^{-}$if $Q_{M}=$ 2. In particular, if $h_{M}^{-}=2 Q_{M}$, then at most two prime ideals are ramified in $M / M^{+}$.

(4) ([Lem1, Theorem 1 (i)]) Let $N=N^{+}(\sqrt{-\delta})$ with $\delta \in N^{+}$totally positive. Assume that $w_{N} \equiv 2 \bmod 4$. We have three mutually exclusive cases:

(a) If $\delta O_{N^{+}}$is not the square of any ideal of $O_{N^{+}}$, then $Q_{N}=1$. In particular, if there is a prime ideal $\mathfrak{p}$ lying above an odd prime $p$ which is ramified in the extension $N / N^{+}$, then $Q_{N}=1$.

(b) If $\delta O_{N^{+}}=\mathfrak{a}^{2}$ for some principal ideal $\mathfrak{a}$ of $O_{N^{+}}$, then $Q_{N}=2$. In particular, if $h_{N^{+}}$is odd and if $\mathrm{N} / \mathrm{N}^{+}$is unramified at all finite primes, then $Q_{N}=2$.

(c) If $\delta O_{N^{+}}=\mathfrak{a}^{2}$ for some non-principal ideal $\mathfrak{a}$ of $O_{N^{+}}$, then $Q_{N}=1$.

(5) If a $C M$-field $N$ has a subfield $M$ which is a CM-field with $Q_{M}=2$, then $Q_{N}=2$ unless $N=N^{+}(\sqrt{-1})$. 
(6) $([\mathrm{W})$ Let $K$ be a $C M$-field of degree $2 n$ over $\mathbb{Q}$. Then

$$
h_{K}^{-}=\frac{w_{K} Q_{K}}{(2 \pi)^{n}} \sqrt{\frac{d_{K}}{d_{K^{+}}}} \frac{\kappa_{K}}{\kappa_{K^{+}}} .
$$

(7) ([Lou10, Theorem 1]) For any totally imaginary number field $K$ of degree $\geq 12$ and root discriminant $\geq 2 \pi^{2}$ we have

$$
\kappa_{K} \geq(1-\beta) /(2 e)
$$

if $\zeta_{K}(\beta) \leq 0$ for some $\beta \in\left[1-\left(2 / \log d_{K}\right), 1\right)$, and

$$
\kappa_{K} \geq 2 /\left(e \log d_{K}\right)
$$

if $\zeta_{K}\left(1-2 / \log d_{K}\right) \leq 0$.

(8) Let $K$ range over the abelian extensions of degree $m$ unramified at all the infinite places of a given $k$. Then there exists a computable constant $\mu_{k} \geq 0$ such that

$$
\kappa_{K} \leq\left(\frac{1}{2(m-1)} \log \left(d_{K} / d_{k}^{m}\right)+2 \mu_{k}\right)^{m-1} \kappa_{k}^{m} .
$$

If $K / k$ is unramified at all the places, then $\kappa_{K} \leq \mu_{k}^{m-1} \kappa_{k}^{m}$. Moreover, if $k$ is a real abelian field of degree $n \geq 2$, then

$$
\mu_{k} \kappa_{k} \leq \frac{\log ^{n} d_{k}}{2^{n+1}(n-1)^{n-1}} .
$$

(9) ([LO2, Proposition 15]) Let $F^{(2)}$ denote the compositum of all the quadratic subfields of a normal number field $F$ of 2-power degree. Then $\zeta_{F} / \zeta_{F^{(2)}}$ is an entire function and $\left(\zeta_{F} / \zeta_{F^{(2)}}\right)(s) \geq 0$ for $s \in(0,1)$.

(10) Let $M=\mathbb{Q}\left(\sqrt{p}_{1}, \sqrt{p}_{2}, \sqrt{p}_{3}\right)$, where the $p_{i}$ 's are the distinct primes $\not 3$ $\bmod$ 4. Set $k=\mathbb{Q}\left(\sqrt{p_{1} p_{2} p_{3}}\right)$. Then

$$
\kappa_{M} \leq \frac{\log ^{7} d_{k}}{2^{4} 3^{6}} .
$$

Moreover, if $\zeta_{M}$ has a real zero $\alpha \in\left(\frac{1}{2}, 1\right)$, then we also have

$$
\kappa_{M} \leq \frac{1-\alpha}{2^{6} 3^{6}} \log ^{8} d_{k} .
$$

(11) Let $k$ be a real abelian number field of degree $n \geq 2$. Then

$$
\kappa_{k} \leq\left(\frac{\log d_{k}}{2(n-1)}\right)^{n-1}
$$

If $k$ is a real quadratic number field and if $\zeta_{k}$ has a real zero $\beta$ in $\left(\frac{1}{2}, 1\right)$, then

$$
\kappa_{k} \leq \frac{1-\beta}{8} \log ^{2} d_{k}
$$

Proof. (5) (See [Ok, Lemma 14]) $Q_{M}=2$ implies $M=M^{+}(\sqrt{-\eta})$ with a totally positive unit $\eta$ in $M^{+}$. Thus $N=N^{+}(\sqrt{-\eta})$, so $Q_{N}=2$ unless $\eta=\alpha^{2}$ for some $\alpha \in N^{+}$.

(8) The first assertion and the second one are Corollary 2 in Lou5]. According to [R1] (see also [R2]) for any primitive even character $\chi$ of conduct $f$ we have $|L(1, \chi)| \leq(\log f) / 2$. Hence, the proofs of [Lou5, Theorem 11] or [Lou9, Theorem 9] now yield the third assertion. 
(10) is derived from [LO2, Proposition 16] and [R1, Corollary 1].

(11) is derived from [Lou4, Corollaries 5A and 7B] and [R1, Corollary 1].

\section{The FIELDS $N$ Which ARE NOT COMPOSITA}

The aim of this section is to prove point (1) of Theorem 1. Throughout this section $N$ denotes a non-abelian normal CM-field of degree 32 which is not a compositum of two normal CM-subfields of degree 16 with the same maximal real subfield. Then the center of its Galois group $G(N / \mathbb{Q})$ must be cyclic. According to [TW] there are 15 non-abelian groups of order 32 whose centers are cyclic: $G_{32 / 17}, G_{32 / 22}, G_{32 / 26}, G_{32 / 31}, G_{32 / 32}$, and $G_{32 / i}$ for $42 \leq i \leq 51$. For each of those 15 groups $G$ we will study whether there exists a CM-field $N$ of class number one with Galois group $G$.

In Subsection 3.1 we will show that if $G(N / \mathbb{Q})$ is neither of type $G_{32 / 26}$ nor $G_{32 / 31}$, then $h_{N}>1$. In Subsection 3.2 we determine all CM-fields of class number one such that their Galois groups are of type $G_{32 / 26}$. In Subsection 3.3 we prove that if $G(N / \mathbb{Q})$ is of type $G_{32 / 31}$, then $h_{N}>1$.

3.1. The fields $N$ with $G(N / \mathbb{Q}) \neq G_{32 / 26}, G_{32 / 31}$. In this subsection we will show that if $G(N / \mathbb{Q}) \neq G_{32 / 26}, G_{32 / 31}$, then $h_{N} \geq 2$.

Lemma 1 ([LO2, Proposition 4]). Let $N$ be a non-abelian normal CM-field of degree $2 n=2^{r} \geq 8$. Assume that $h_{N}^{-}$is odd and $N$ is cyclic of degree $n$ over a quadratic subfield $k$. Then $k$ is real and $N$ is a dihedral CM-field.

Proposition 1. If $G(N / \mathbb{Q})$ has an element of order 16 , then $h_{N}^{-} \geq 2$. In particular, if $G(N / \mathbb{Q}) \in\left\{G_{32 / 22}, G_{32 / 50}, G_{32 / 51}\right\}$, then $h_{N}^{-}$is even, and if $G(N / \mathbb{Q})=$ $G_{32 / 49}\left(=D_{32}\right)$, then $h_{N}^{-} \geq 2$.

Proof. Follows from Lemma 1 and [Lef, Theorem 1.1].

Proposition 2. If $G\left(N^{+} / \mathbb{Q}\right)$ is isomorphic to $C_{2} \times C_{2} \times C_{2} \times C_{2}$, then $h_{N}$ is even. In particular, if $G(N / \mathbb{Q}) \in\left\{G_{32 / 42}, G_{32 / 43}\right\}$, then $h_{N}$ is even.

Proof. Suppose the contrary. Since $h_{N}$ is odd and $N$ is a non-abelian number field, $G\left(N^{+}\right)=N^{+}$. Hence, $N^{+}=\mathbb{Q}\left(\sqrt{p}_{1}, \sqrt{p}_{2}, \sqrt{p}_{3}, \sqrt{p}_{4}\right)$ where $p_{i}$ 's are primes with $\not \equiv 3 \bmod 4$. Then $N^{+}$is the Hilbert 2-class field of $k=\mathbb{Q}\left(\sqrt{p_{1} p_{2} p_{3} p_{4}}\right)$ and the 2-class field tower of $k$ has length one. According to [BLS, Corollary 2] the 2-rank of $c l(k)$ is $\leq 2$ which contradicts the fact that $G\left(N^{+} / k\right) \simeq \operatorname{cl}(k) \simeq C_{2} \times C_{2} \times C_{2}$.

Proposition 3. If $G(N / \mathbb{Q}) \in\left\{G_{32 / 44}, G_{32 / 46}\right\}$, then $h_{N}^{-}$is even.

Proof. We will show that $N=M_{16.1} M_{16.2}$ is a compositum of two isomorphic non-normal CM-subfields with $M_{16.1}^{+}=M_{16.2}^{+}$, and that one of these two fields say, $M_{16.1}=M_{8.1} M_{8.2}$, is a compositum of two isomorphic non-normal octic CMsubfields with $M_{8.1}^{+}=M_{8.2}^{+}$. Hence, $h_{N}^{-}=\frac{Q_{N}}{2}\left(\frac{h_{M_{16.1}}^{-}}{Q_{M_{16.1}}}\right)^{2}$ and $h_{M_{16.1}}^{-}=\frac{Q_{M_{16.1}}}{2}\left(\frac{h_{M_{8.1}}^{-}}{Q_{M_{8.1}}}\right)^{2}$, by (B), which implies that $h_{N}^{-}$is always even. Write $G_{32 / 44}=\left\langle a, b, c: a^{8}=\right.$ $\left.b^{2}=c^{2}=1, a b=b a^{-1}, a c=c a^{5}, b c=c b\right\rangle$ and $G_{32 / 46}=\left\langle a, b, c, d: a^{2}=b^{2}=\right.$ $\left.c^{2}=d^{4}=1, b d=d a b, c d=d a b c, a b=b a, a c=c a, a d=d a, b c=c b\right\rangle$. Note that $C\left(G_{32 / 44}\right)=\left\langle a^{4}\right\rangle$ and $C\left(G_{32 / 46}\right)=\langle a\rangle$. When $G(N / \mathbb{Q})=G_{32 / 44}$, set $M_{16.1}=\langle c\rangle^{\prime}, M_{16.2}=\left\langle a^{4} c\right\rangle^{\prime}, M_{8.1}=\langle b, c\rangle^{\prime}$, and $M_{8.2}=\left\langle a^{6} b a^{-6}, c\right\rangle^{\prime}$. We have $a^{4} c=a c a^{-1}, M_{16.1}^{+}=M_{16.2}^{+}=\left\langle a^{4}, c\right\rangle^{\prime},\left\langle a^{6} b a^{-6}, c\right\rangle=a^{6}\langle b, c\rangle a^{-6}, M_{8.1}^{+}=M_{8.2}^{+}=$ 
$\left\langle a^{4}, b, c\right\rangle^{\prime}$. When $G(N / \mathbb{Q})=G_{32 / 46}$, set $M_{16.1}=\langle b\rangle^{\prime}, M_{16.2}=\left\langle d b d^{-1}\right\rangle^{\prime}, M_{8.1}=$ $\langle b, c\rangle^{\prime}$, and $M_{8.2}=\langle b, a c\rangle^{\prime}$. Then $a c=d^{2} c d^{-2}, M_{16.1}^{+}=M_{16.2}^{+}=\langle a, b\rangle^{\prime}$, and $M_{8.1}^{+}=M_{8.2}^{+}=\langle a, b, c\rangle^{\prime}$.

Lemma 2 (Chebotarev's Monodromy Theorem). Let $k \subseteq K$ be two number fields such that $K / \mathbb{Q}$ and $k / \mathbb{Q}$ are normal, and $K / k$ is unramified at all the finite places. Let $m$ be the least positive integer such that $g^{m}=1$ for every $g \in G(k / \mathbb{Q})$. Then the Galois group $G(K / \mathbb{Q})$ is generated by the elements $\sigma$ which are not contained in the subgroup $G(K / k)$ and at the same time satisfy $\sigma^{m}=1$.

Proof. See [Lem2, Corollary 1].

Proposition 4. If $G(N / \mathbb{Q}) \in\left\{G_{32 / 45}, G_{32 / 47}, G_{32 / 48}\right\}$, then $h_{N}^{-}$is even.

Proof. Write $G_{32 / 45}=\langle a, b, c| a^{8}=b^{2}=1, c^{2}=a^{4}, a b=b a^{5}, a c=c a^{-1}, b c=$ $c b\rangle, G_{32 / 47}=\left\langle a, b, c \mid a^{8}=b^{2}=c^{2}=1, a b=b a^{5}, a c=c b a, b c=c b\right\rangle$, and $G_{32 / 48}=\left\langle a, b, c \mid a^{8}=b^{2}=1, c^{2}=a^{4}, a b=b a^{5}, a c=c b a, b c=c b\right\rangle$. Note that the centers of these three groups are $\left\langle a^{4}\right\rangle$, so $N^{+}=\left\langle a^{4}\right\rangle^{\prime}$. Assume that $G(N / \mathbb{Q}) \in\left\{G_{32 / 45}, G_{32 / 47}, G_{32 / 48}\right\}$. Since $N$ is a compositum of two isomorphic CM-subfields $\langle b\rangle^{\prime}$ and $\left\langle a b a^{-1}\right\rangle^{\prime}$ with the same maximal real subfield $\left\langle a^{4}, b\right\rangle^{\prime}$, according to Theorem 2, points (1) and (3), if $h_{N}^{-}$is odd, then $N / N^{+}$is unramified at all the finite places, hence so is $N /\left\langle a^{2}\right\rangle^{\prime}$. Note that $\left\langle a^{2}\right\rangle$ is a normal subgroup of $G(N / \mathbb{Q})$. We will show that $N /\left\langle a^{2}\right\rangle^{\prime}$ cannot be unramified at all the finite places.

(1) If $G(N / \mathbb{Q})=G_{32 / 45}$, then $G(N / \mathbb{Q}) /\left\langle a^{2}\right\rangle \simeq C_{2} \times C_{2} \times C_{2}$. We observe that the subgroup generated by the elements of order 2 in $G(N / \mathbb{Q})$ is $\langle b, a c b, c a b\rangle$ which is a group of order 16 isomorphic to the group of type 16/8 designated in TW] (or named $G_{9}$ in [JL]). According to Lemma 2, $N /\left\langle a^{2}\right\rangle^{\prime}$ cannot be unramified at all the finite places and $h_{N}^{-}$is even.

(2) If $G(N / \mathbb{Q})=G_{32 / 47}$ or $G_{32 / 48}$, then $G(N / \mathbb{Q}) /\left\langle a^{2}\right\rangle \simeq D_{8}$. We observe that the subgroup generated by the elements of order 2 or 4 in $G(N / \mathbb{Q})$ is $\left\langle a^{2}, b, c\right\rangle$ which is isomorphic to $D_{8} \times C_{2}$ or $Q_{8} \times C_{2}$ according as $G(N / \mathbb{Q})=G_{32 / 47}$ or $G_{32 / 48}$. By the same argument as in (1), it follows that $h_{N}^{-}$is even.

Lemma 3. Let $K \subset K^{\prime} \subset L$ be the number fields such that $L / K$ is a Galois extension. Let $K^{a b}$ ( $K^{\prime a b}$, respectively) be the maximal extension of $K\left(K^{\prime}\right.$, resp.) contained in $L$ which is abelian over $K\left(K^{\prime}\right.$, resp.). Let $\mathfrak{m}$ and $\mathfrak{m}^{\prime}$ be the conductors of $K^{a b} / K$ and $K^{\prime a b} / K^{\prime}$, respectively, $I_{\mathfrak{m}}$ the group of fractional ideals of $K$ coprime to $\mathfrak{m}$, and let $I_{\mathfrak{m}^{\prime}}$ be that of $K^{\prime}$ coprime to $\mathfrak{m}^{\prime}$. Then the following diagram is commutative:

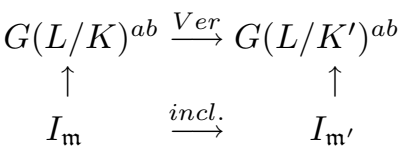

Here, Ver means the transfer map.

Proof. See the functorial properties of the reciprocity map in [S, Ch. XI §3]. (See also [Mar or [N, Ch. II and IV].)

Proposition 5. If $G(N / \mathbb{Q}) \in\left\{G_{32 / 17}, G_{32 / 32}\right\}$, then $h_{N}^{-}$is even.

Proof. Write $G_{32 / 17}=\left\langle a, b, c \mid a^{8}=b^{2}=c^{2}=1, a b=b a, a c=c a, b c=c a^{4} b\right\rangle$ and $G_{32 / 32}=\left\langle a, b \mid a^{8}=1, a^{4}=b^{4}, a b=b a^{-1}\right\rangle$. Note that $C\left(G_{32 / 17}\right)=\langle a\rangle$ and $C\left(G_{32 / 32}\right)=\left\langle b^{2}\right\rangle$. 
(a) Assume that $G(N / \mathbb{Q})=G_{32 / 17}$. The field $N$ is a compositum of two isomorphic CM-subfields $\langle b\rangle^{\prime}$ and $\left\langle c b c^{-1}\right\rangle^{\prime}$ with the same maximal real subfield $\left\langle a^{4}, b\right\rangle^{\prime}$. Suppose that $h_{N}^{-}$is odd. By Theorem 2, points (1) and (3), $N /\left\langle a^{4}\right\rangle^{\prime}$ is unramified at all the finite places and so is $N /\langle a\rangle^{\prime}$. Since the ring of integers $\mathbb{Z}$ is principal, according to Lemma 3 the transfer map Ver $: G_{32 / 17}^{a b} \rightarrow\langle a\rangle$ would be trivial. However, $\operatorname{Ver}\left(a D\left(G_{32 / 17}\right)\right)=a^{4}$, which is a contradiction. Hence, $h_{N}^{-}$is always even.

(b) Assume that $G(N / \mathbb{Q})=G_{32 / 32}$. The field $N$ is a compositum of two isomorphic CM-subfields $\left\langle a^{2} b^{2}\right\rangle^{\prime}$ and $\left\langle a^{6} b^{2}\right\rangle^{\prime}$ with the same maximal real subfield $\left\langle a^{2} b^{2}, a^{4}\right\rangle^{\prime}$. (Note that $a^{6} b^{2}=b a^{2} b^{2} b^{-1}$.) Suppose that $h_{N}^{-}$is odd. By Theorem 2, points (1) and (3), $N /\left\langle b^{4}\right\rangle^{\prime}$ is unramified at all the finite places and so is $N /\langle b\rangle^{\prime}$. Hence, Ver $: G_{32 / 32}^{a b} \rightarrow\langle b\rangle$ would be trivial. However, $\operatorname{Ver}\left(a D\left(G_{32 / 32}\right)\right)=b^{4}$, which is a contradiction. Hence, $h_{N}^{-}$is always even.

3.2. The fields $N$ with $G(N / \mathbb{Q})=G_{32 / 26}$. Write $G_{32 / 26}=\langle a, b, c| a^{8}=b^{2}=$ $\left.c^{2}=1, a b=b a, a c=c a^{-1}, b c=c a^{4} b\right\rangle$. Since $C\left(G_{32 / 26}\right)=\left\langle a^{2} b\right\rangle \simeq C_{4}$, the complex conjugation is $a^{4}$ and $G\left(N^{+} / \mathbb{Q}\right) \simeq D_{8} \times C_{2}$. We will prove the following:

Proposition 6. $G(N / \mathbb{Q})=G_{32 / 26}$ and $h_{N}=1$ if and only if

$$
N=\operatorname{Hil}_{\text {nar }}(\mathbb{Q}(\sqrt{5 \cdot 29}, \sqrt{2})) .
$$

We proceed as follows. First, we prove that if $h_{N}=1$, then

$$
N=H i l_{\text {nar }}\left(\mathbb{Q}\left(\sqrt{p_{1} p_{2}}, \sqrt{p_{3}}\right)\right),
$$

where $p_{i}$ 's are primes $\not \equiv 3 \bmod 4$. Second, we find a $C$ such that if $d_{N} \geq C$, then $h_{N}^{-}>1$. Third, using Hecke $L$-functions we compute $h_{N}^{-}$for the fields $N$ with $d_{N}<C$ and prove Proposition 6 .

Lemma 4. (1) If $h_{N}^{-}$is odd, then $Q_{N}=2$ and $N$ has a unique real quadratic subfield $k$ such that $G(N / k) \simeq Q_{16}$, and $N / k$ is unramified at all the finite places. Moreover, $d_{k}=d_{1} d_{2} d_{3}$ is the product of three distinct discriminants of real quadratic fields and

(i) $\left(d_{i}, d_{j}\right)=1$ for $i \neq j$;

(ii) $\left(\frac{d_{1}}{p_{2}}\right)=\left(\frac{d_{2}}{p_{1}}\right)=1$ for all primes $p_{1} \mid d_{1}$ and $p_{2} \mid d_{2}$;

(iii) $G\left(N^{+} / k\left(\sqrt{d_{3}}\right)\right)=C_{4}$ and $G\left(N^{+} / k\left(\sqrt{d_{j}}\right)\right)=C_{2} \times C_{2}$ for $j=1,2$.

(2) If $h_{N}=1$, then $k=\mathbb{Q}\left(\sqrt{d_{1} d_{2} d_{3}}\right)$ and these $d_{i}$ 's are primes $d_{i} \neq \equiv \bmod 4$. In addition, $N=H_{i l_{\text {nar }}}\left(\mathbb{Q}\left(\sqrt{d_{1} d_{2}}, \sqrt{d}_{3}\right)\right)$.

Proof. (1) Assume that $h_{N}^{-}$is odd. Since $a^{4} b=c b c^{-1}, N$ is a compositum of two isomorphic CM-subfields $\langle b\rangle^{\prime}$ and $\left\langle a^{4} b\right\rangle^{\prime}$ with the same maximal real subfield $\left\langle a^{4}, b\right\rangle^{\prime}$. By Theorem 2, $Q_{N}=2$ and $N / N^{+}$is unramified at all the finite places. Since $a^{4}$ is the unique element of order 2 in $\langle a, c b\rangle \simeq Q_{16}, N /\langle a, c b\rangle^{\prime}$ is unramified at all the finite places. Set $k=\langle a, c b\rangle^{\prime}$. The remaining statements follow from Lem2, Theorem 2].

(2) Let $M=\left\langle a^{2}\right\rangle^{\prime}$. Then $G(M / \mathbb{Q})=C_{2} \times C_{2} \times C_{2}$ and $M$ is the maximal normal subfield of $N^{+}$which is an abelian extension of $k$. If $h_{N}=1$, then $M=$ $\operatorname{Hil}(k)=G(k)$. Hence $d_{i}$ 's are primes $d_{i} \not \equiv 3 \bmod 4$ and $M=\mathbb{Q}\left(\sqrt{d}_{1}, \sqrt{d}_{2}, \sqrt{d}_{3}\right)$. Let $K=\langle a\rangle^{\prime}$. If $h_{N}=1$, then $N^{+}=\operatorname{Hil}(K)$ and $N=\operatorname{Hil}_{\text {nar }}(K)$. Note that $G\left(N / k\left(\sqrt{d}_{1}\right)\right) \simeq G\left(N / k\left(\sqrt{d}_{2}\right)\right) \simeq Q_{8}$. Moreover, according to (1) point (iii) we have $K=k\left(\sqrt{d}_{3}\right)$. 
From now on $p_{1}, p_{2}$, and $p_{3}$ denote distinct primes $\not \equiv 3 \bmod 4$ satisfying $\left(\frac{p_{2}}{p_{1}}\right)=$ $\left(\frac{p_{1}}{p_{2}}\right)=1$. Write $K=\langle a\rangle^{\prime}=\mathbb{Q}\left(\sqrt{p_{1} p_{2}}, \sqrt{p_{3}}\right), k=\langle a, c b\rangle^{\prime}=\mathbb{Q}\left(\sqrt{p_{1} p_{2} p_{3}}\right)$, and $M=\left\langle a^{2}\right\rangle^{\prime}=\mathbb{Q}\left(\sqrt{p}_{1}, \sqrt{p}_{2}, \sqrt{p}_{3}\right)$.

Lemma 5. Assume that $h_{N}=1$.

(i) $\mathbb{Q}\left(\sqrt{p}_{i}\right)$ has class number one for $i=1,2$, and 3 .

(ii) $\operatorname{cl}\left(\mathbb{Q}\left(\sqrt{p_{1} p_{2}}\right)\right) \simeq C_{4}, \operatorname{cl}\left(\mathbb{Q}\left(\sqrt{p_{1} p_{3}}\right)\right) \simeq \operatorname{cl}\left(\mathbb{Q}\left(\sqrt{p_{2} p_{3}}\right)\right) \simeq C_{2}$.

Proof. (i) The class number of $\mathbb{Q}\left(\sqrt{p}_{i}\right)$ is odd and $\operatorname{Hil}\left(\mathbb{Q}\left(\sqrt{p}_{i}\right)\right)$ lies in $N$. Hence the class number of $\mathbb{Q}\left(\sqrt{p}_{i}\right)$ must be equal to one.

(ii) Since $G(N / k)=\langle a, c b\rangle$ is the dicyclic group of order 16, it has two quaternion subgroups of order 8: $\left\langle a^{2}, c b\right\rangle$ and $\left\langle a^{2}, a c b\right\rangle$. Changing $p_{1}$ to $p_{2}$ if necessary we may assume that $\left\langle a^{2}, a c b\right\rangle^{\prime}=\mathbb{Q}\left(\sqrt{p_{1} p_{2} p_{3}}, \sqrt{p_{1}}\right)$ and $\left\langle a^{2}, c b\right\rangle^{\prime}=\mathbb{Q}\left(\sqrt{p_{1} p_{2} p_{3}}, \sqrt{p_{2}}\right)$. Note that the element $a b$ is of order 8 and $(a b)^{2}=a^{2}$. The fixed field of $\langle a b\rangle$ lies in $\left\langle a^{2}\right\rangle^{\prime}=\mathbb{Q}\left(\sqrt{p}_{1}, \sqrt{p}_{2}, \sqrt{p}_{2}\right)$ and $N /\langle a b\rangle^{\prime}$ is unramified at all the finite places. Hence, $\mathbb{Q}\left(\sqrt{p}_{1}, \sqrt{p}_{2}, \sqrt{p}_{3}\right) /\langle a b\rangle^{\prime}$ is unramified and $\langle a b\rangle^{\prime}=\mathbb{Q}\left(\sqrt{p_{1} p_{2}}, \sqrt{p_{2} p_{3}}\right)$. Thus, $\langle a, b\rangle^{\prime}=\mathbb{Q}\left(\sqrt{p_{1} p_{2}}\right),\langle a b, a c b\rangle^{\prime}=\mathbb{Q}\left(\sqrt{p_{2} p_{3}}\right)$, and $\langle a b, c b\rangle^{\prime}=\mathbb{Q}\left(\sqrt{p_{1} p_{3}}\right)$. Since $G\left(N^{+} / \mathbb{Q}\left(\sqrt{p_{1} p_{2}}\right)\right) \simeq C_{4} \times C_{2}$ and $\operatorname{Hil}\left(\mathbb{Q}\left(\sqrt{p_{1} p_{2}}\right)\right)$ is contained in $N^{+}, \operatorname{Hil}\left(\mathbb{Q}\left(\sqrt{p_{1} p_{2}}\right)\right)$ must be the fixed field of the inertia group of a prime ideal in $N^{+}$lying above $p_{3}$. Note that the inertia group is of order 2 and all intermediate fields between $N^{+}$and $\mathbb{Q}\left(\sqrt{p_{1} p_{2}}\right)$ are normal extensions over $\mathbb{Q}$. Because the 2-rank of $\operatorname{cl}\left(\mathbb{Q}\left(\sqrt{p_{1} p_{2}}\right)\right)$ is equal to $1, \operatorname{Hil}\left(\mathbb{Q}\left(\sqrt{p_{1} p_{2}}\right)\right)$ is one of the two cyclic quartic extensions of $\mathbb{Q}\left(\sqrt{p_{1} p_{2}}\right)$ contained in $N^{+}$and $\operatorname{cl}\left(\mathbb{Q}\left(\sqrt{p_{1} p_{2}}\right)\right) \simeq C_{4}$. Similarly, we verify that $\operatorname{Hil}\left(\mathbb{Q}\left(\sqrt{p_{2} p_{3}}\right)\right)=\mathbb{Q}\left(\sqrt{p}_{2}, \sqrt{p}_{3}\right)$ and $\operatorname{Hil}\left(\mathbb{Q}\left(\sqrt{p_{1} p_{3}}\right)\right)=\mathbb{Q}\left(\sqrt{p}_{1}, \sqrt{p}_{3}\right)$. Since $h_{N}=h_{N^{+}}=1, \operatorname{Hil}\left(\mathbb{Q}\left(\sqrt{p_{2} p_{3}}\right)\right)$ and $\operatorname{Hil}\left(\mathbb{Q}\left(\sqrt{p_{1} p_{3}}\right)\right)$ are contained in $N^{+}$. Note that $G\left(N^{+} / \mathbb{Q}\left(\sqrt{p_{2} p_{3}}\right)\right) \simeq\langle a b, a c b\rangle /\left\langle a^{4}\right\rangle \simeq D_{8}$ and $G\left(N^{+} / \mathbb{Q}\left(\sqrt{p_{1} p_{3}}\right)\right) \simeq$ $\langle a b, c b\rangle /\left\langle a^{4}\right\rangle \simeq D_{8}$. The field $\mathbb{Q}\left(\sqrt{p}_{2}, \sqrt{p}_{3}\right)$ (respectively, $\left.\mathbb{Q}\left(\sqrt{p}_{1}, \sqrt{p}_{3}\right)\right)$ is the unique intermediate field between $N^{+}$and $\mathbb{Q}\left(\sqrt{p_{2} p_{3}}\right)$ (respectively, $\mathbb{Q}\left(\sqrt{p_{1} p_{3}}\right)$ ) that is an unramified and normal over $\mathbb{Q}\left(\sqrt{p_{2} p_{3}}\right)$ (respectively, $\mathbb{Q}\left(\sqrt{p_{1} p_{3}}\right)$ ). It follows that $\operatorname{cl}\left(\mathbb{Q}\left(\sqrt{p_{2} p_{3}}\right)\right) \simeq \operatorname{cl}\left(\mathbb{Q}\left(\sqrt{p_{1} p_{3}}\right)\right) \simeq C_{2}$.

From now on we assume that $N$ is unramified over $k=\mathbb{Q}\left(\sqrt{p_{1} p_{2} p_{3}}\right)$ at all the finite places and $Q_{N}=2$. Hence $d_{N}=d_{k}^{16}$ and $d_{N^{+}}=d_{k}^{8}$. We first show that if $d_{k} \geq 9.1 \times 10^{5}$, then $h_{N}^{-}>1$. Set $\beta_{N}=1-\left(2 / \log d_{N}\right)$.

(i) Assume that $\zeta_{N}\left(\beta_{N}\right) \leq 0$. By Theorem 2, points (7), (8), and (10),

$$
\kappa_{N} \geq \frac{1}{2^{3} e \log d_{k}}
$$

and

$$
\kappa_{N^{+}} \leq \mu_{M} \kappa_{M}^{2} \leq \frac{2^{3} \log ^{15} d_{k}}{3^{6} 7^{7}}
$$

Hence,

$$
h_{N}^{-} \geq \frac{3^{6} 7^{7}}{2^{20} e \pi^{16}} \frac{d_{k}^{4}}{\log ^{16} d_{k}}
$$

and $h_{N}^{-}>1$ if $d_{k} \geq 9.1 \times 10^{5}$.

(ii) Assume that $\zeta_{N}\left(\beta_{N}\right)>0$. Since $N^{(2)}=M$, by Theorem 2, point (9), we have $\zeta_{M}\left(\beta_{N}\right)>0$ and there is a positive real number $\beta$ such that $\beta_{N}<\beta<1$ and 


$$
\begin{array}{r}
\zeta_{M}(\beta)=0 . \text { Hence, } \zeta_{N^{+}}(\beta)=\zeta_{N}(\beta)=0, \kappa_{N} \geq(1-\beta) /(2 e), \\
\kappa_{N^{+}} \leq \mu_{M} \kappa_{M}^{2} \leq \frac{2(1-\beta) \log ^{16} d_{k}}{3^{6} 7^{7}} .
\end{array}
$$

and we end up with a lower bound for $h_{N}^{-}$which is $2^{4}$ times better than (D). Thus (D) is always valid and we conclude that $h_{N}^{-}>1$ if $d_{k} \geq 9.1 \times 10^{5}$.

Now, there are 510 triplets $\left(p_{1}, p_{2}, p_{3}\right)$ such that $d_{k} \leq 9.1 \times 10^{5}$ and $p_{1}, p_{2}$, and $p_{3}$ satisfy Lemmas 4 and 5 . For those 510 triplets $\left(p_{1}, p_{2}, p_{3}\right)$ we compute explicitly $\kappa_{M}$ (see [Lou5, Section 4.2]) and improve our upper bound for $\kappa_{N^{+}}$:

$$
\kappa_{N^{+}} \leq \kappa_{M} \cdot \mu_{M} \kappa_{M} \leq \kappa_{M} \frac{2^{7} \log ^{8} d_{k}}{7^{7}}
$$

We improve the lower bound for $h_{N}^{-}$as follows. If $\zeta_{N}\left(\beta_{N}\right) \leq 0$, then

$$
h_{N}^{-} \geq \frac{7^{7}}{2^{24} e \pi^{16}} \frac{d_{k}^{4}}{\kappa_{M} \log ^{9} d_{k}} .
$$

If $\zeta_{N}\left(\beta_{N}\right)>0$, then there is a positive real number $\beta \in\left(\beta_{N}, 1\right)$ such that $\zeta_{M}(\beta)=$ $\zeta_{N}(\beta)=0$. Hence,

where

$$
\kappa_{N} \geq \frac{(1-\beta)}{2 e} \geq \frac{4}{e B_{N^{(2)}}},
$$

$$
B_{N^{(2)}}=\max _{\substack{F \subset N^{(2)} \\[F:(])=2}} \frac{\log ^{2} d_{F}}{L\left(1, \chi_{F}\right)}
$$

and $\chi_{F}(p)=\left(\frac{d_{F}}{p}\right)$ denotes the corresponding quadratic character of $F$. In fact, $\zeta_{M}(\beta)=0$ implies that there exists a subfield $F \subset M=N^{(2)}$ with $[F: \mathbb{Q}]=2$ such that $\zeta_{F}(\beta)=0$. Hence,

$$
1 \leq \frac{1-\beta}{8} \frac{\log ^{2} d_{F}}{L\left(1, \chi_{F}\right)} \leq \frac{1-\beta}{8} B_{N^{(2)}}
$$

by $(\mathrm{C})$. Thus

$$
h_{N}^{-} \geq \frac{7^{7}}{2^{19} e \pi^{16}} \frac{d_{k}^{4}}{\kappa_{M} B_{N^{(2)}} \log ^{8} d_{k}} .
$$

For these 510 triplets $\left(p_{1}, p_{2}, p_{3}\right)$ we compute two lower bounds for $h_{N}^{-}$in (E) and (F) respectively, and then take the worse one. There are 467 out of these 510 triplets with $h_{N}^{-}>1$. Finally, only 19 out of the remaining $43(=510-467)$ triplets $\left(p_{1}, p_{2}, p_{3}\right)$ satisfy $\operatorname{cl}(K) \simeq C_{4}$, where $K=\mathbb{Q}\left(\sqrt{p_{1} p_{2}}, \sqrt{p_{3}}\right)$ (use [KT]). For these 19 triplets $\left(p_{1}, p_{2}, p_{3}\right)$ we construct the primitive Hecke characters $\chi$ of order 8 on the narrow class group of $K$ and evaluate $L(0, \chi)$ using the technique developed in Lou7 and Lou8, where $L(s, \chi)$ is the Hecke $L$-function. According to Lou8 we have

$$
h_{N}^{-}=Q_{N} w_{N} N_{\mathbb{Q}(\sqrt{2}, i) / \mathbb{Q}}\left(\frac{1}{2^{4}} L(0, \chi)\right) .
$$

We verify that there is one and only one field $K$ that has $h_{N}^{-}=1: K=\mathbb{Q}(\sqrt{5 \cdot 29}, \sqrt{2})$ and $L(0, \chi)=16+8 \sqrt{2}$. Using the package $\mathrm{KT}$ we verify that

$$
G\left(\operatorname{Hil}_{\text {nar }}(\mathbb{Q}(\sqrt{5 \cdot 29}, \sqrt{2})) / \mathbb{Q}\right) \simeq G_{32 / 26}
$$

and $h_{H i l(\mathbb{Q}(\sqrt{5 \cdot 29}, \sqrt{2}))}=1$. This completes the proof of Proposition 6 . 
3.3. The fields $N$ with $G(N / \mathbb{Q})=G_{32 / 31}$. Write $G_{32 / 31}=\langle a, b, c| a^{4}=b^{4}=$ $\left.c^{2}=1, a b=b a, a c=c a, b c=c a b^{-1}\right\rangle$. Since $C\left(G_{32 / 31}\right)=\langle a\rangle$, the complex conjugation is $a^{2}$ and $N^{+}=\left\langle a^{2}\right\rangle^{\prime}$. The aim of this subsection is to prove:

Proposition 7. If $G(N / \mathbb{Q})=G_{32 / 31}$, then $h_{N}>1$.

Before starting the proof we observe that $k_{1}=\left\langle c b, b^{2}\right\rangle^{\prime}, k_{2}=\langle a, b\rangle^{\prime}$, and $k_{3}=$ $\left\langle a, b^{2}, c\right\rangle^{\prime}$ are the only quadratic subfields of $N$. Note that $G\left(N^{+} / k_{1}\right)=C_{4} \times$ $C_{2}, G\left(N^{+} / k_{2}\right)=C_{4} \times C_{2}$, and $G\left(N^{+} / k_{3}\right)=C_{2} \times C_{2} \times C_{2}$.

Lemma 6. If $G(N / \mathbb{Q})=G_{32 / 31}$ and $h_{N}^{-}$is odd, then every prime divisor of $d_{k_{3}}$ divides $d_{k_{1}}$ and is not equal to 3 modulo 4 . Such primes are the unique primes having ramification index 4 in $N / \mathbb{Q}$.

Proof. Since $c b^{2} c^{-1}=a^{2} b^{2}, N$ is a compositum of two isomorphic CM-subfields $\left\langle b^{2}\right\rangle^{\prime}$ and $\left\langle a^{2} b^{2}\right\rangle^{\prime}$ with the same maximal real subfield $\left\langle a^{2}, b^{2}\right\rangle^{\prime}$. According to Theorem 2 the fact that $h_{N}^{-}$is odd implies that $N / N^{+}$is unramified at all the finite places. Let $L_{3}=\left\langle a b^{2}, a c\right\rangle^{\prime}$. Since $\left\langle a b^{2}, a c\right\rangle \simeq Q_{8}$ and $\left(a b^{2}\right)^{2}=a^{2}, N / L_{3}$ is unramified at all the finite places. The only prime divisors of $d_{N}$ are hence those of $d_{L_{3}}$. The subgroup $\left\langle a b^{2}, a c\right\rangle$ is normal in $G_{32 / 31}$ with $G_{32 / 31} /\left\langle a b^{2}, a c\right\rangle \simeq C_{4}$. Since $\left\langle a b^{2}, a c\right\rangle \subset\left\langle a, b^{2}, c\right\rangle$ and $k_{3} \subset L_{3}$, every prime divisor $p$ of $d_{k_{3}}$ is totally ramified in the cyclic extension $L_{3} / \mathbb{Q}$. Hence, $p \neq 3 \bmod 4$ and the ramification index of $p$ in $N$ is equal to 4 . Let $L_{1}=\langle c b\rangle^{\prime}$. As $(c b)^{2}=a$ and $L_{1} \subset N^{+}, N / L_{1}$ is unramified at all the finite places. Hence, $p$ is totally ramified in $L_{1}$ and $p \mid d_{k_{1}}$.

Remark 1. The fact that if $h_{N}^{-}$is odd, then $N / L_{3}$ is unramified at all the finite places will be used in the sequel.

The proof of Proposition 7 consists of four parts.

Part 1: We claim that if $d_{L_{3}} \geq 8.7 \times 10^{14}$, then $h_{N}^{-}>1$.

Proof. Set $\beta_{N}=1-2 / \log d_{N}$.

(i) Assume that $\zeta_{N}\left(\beta_{N}\right) \leq 0$. By Theorem 2

$$
\kappa_{N} \geq \frac{2}{e \log d_{N}}=\frac{1}{4 e \log d_{L_{3}}}
$$

and

Hence,

$$
\kappa_{N^{+}} \leq \kappa_{L_{3}} \cdot\left(\mu_{L_{3}} \kappa_{L_{3}}\right)^{3} \leq \frac{\log ^{15} d_{L_{3}}}{2^{18} 3^{12}}
$$

$$
h_{N}^{-} \geq \frac{2^{2} 3^{12} d_{L_{3}}^{2}}{e \pi^{16} \log ^{16} d_{L_{3}}}
$$

and $h_{N}^{-}>1$ if $d_{L_{3}} \geq 6.1 \times 10^{12}$.

(ii) Assume that $\zeta_{N}\left(\beta_{N}\right)>0$. Since $G_{32 / 31}^{a b} \simeq C_{4} \times C_{2}, N^{(2)}$ is the biquadratic bicyclic subfield of $N$ containing $k_{1}, k_{2}$, and $k_{3}$, i.e., $N^{(2)}=L_{4}=\left\langle a, b^{2}\right\rangle^{\prime}\left(\left\langle a, b^{2}\right\rangle=\right.$ $\left.\left\langle c b, b^{2}\right\rangle \cap\langle a, b\rangle \cap\left\langle a, b^{2}, c\right\rangle\right)$. According to Theorem 2, point (9), $\zeta_{N^{(2)}}\left(\beta_{N}\right)>0$, which yields $\zeta_{N^{(2)}}(\beta)=0$ for some $\beta \in\left(\beta_{N}, 1\right)$. Thus $\zeta_{k_{i}}(\beta)=0$ for some $i=1,2$, or 3 . Hence, $\kappa_{N} \geq \frac{(1-\beta)}{2 e}$ and $\kappa_{k_{i}}>\frac{1-\beta}{8} \log ^{2} d_{k_{i}}$. By Theorem 2, point (8), we have

$$
\kappa_{N^{+}} \leq\left(\frac{1}{14} \log \left(d_{N^{+}} / d_{k_{i}}^{8}\right) \kappa_{k_{i}}+2 \mu_{k_{i}} \kappa_{k_{i}}\right)^{7} \kappa_{k_{i}},
$$


and $\mu_{k_{i}} \kappa_{k_{i}} \leq \frac{1}{8} \log ^{2} d_{k_{i}}$. Since $d_{k_{i}}^{2} \leq d_{L_{3}}$ and $d_{N^{+}} / d_{k_{i}}^{8}=\left(d_{L_{3}} /\left(d_{k_{i}}\right)^{2}\right)^{4}<d_{L_{3}}^{4}$, we get

$$
\begin{gathered}
\kappa_{N^{+}} \leq \frac{3^{7} 5^{7}}{2^{33} 7^{7}}(1-\beta) \log ^{16} d_{L_{3}}, \\
h_{N}^{-} \geq \frac{2^{18} 7^{7}}{3^{7} 5^{7} e \pi^{16}} \frac{d_{L_{3}}{ }^{2}}{\log ^{16} d_{L_{3}}},
\end{gathered}
$$

and $h_{N}^{-}>1$ if $d_{L_{3}} \geq 8.7 \times 10^{14}$. (However, we can obtain $\epsilon_{N} \geq 0.978$ and $h_{N}^{-}>1$ if $d_{L_{3}} \geq 5.6 \times 10^{14}$ using [Lou1, Proposition A].)

Part 2: We claim that

$$
h_{N}^{-}=\frac{Q_{N}}{8}\left(\frac{h_{K_{8.1}}^{-}}{Q_{K_{8.1}}} \frac{h_{K_{8.2}}^{-}}{Q_{K_{8.2}}}\right)^{2},
$$

where $K_{8.1}=\langle b\rangle^{\prime}$ and $K_{8.2}=\left\langle a^{2} b\right\rangle^{\prime}$.

Proof. Since $G\left(N / k_{2}\right)=\langle a, b\rangle \simeq C_{4} \times C_{4}$, there are four octic CM-subfields of $N$ containing $k_{2}: K_{8.1}=\langle b\rangle^{\prime}, K_{8.2}=\left\langle a^{2} b\right\rangle^{\prime}, L_{8.1}=\left\langle a^{3} b\right\rangle^{\prime}$ and $L_{8.2}=\langle a b\rangle^{\prime}$. Note that $c b c^{-1}=a b^{3}$ and $c a^{2} b c^{-1}=(a b)^{-1}$. Then $K_{8.1}^{+}=K_{8.2}^{+}=\left\langle a^{2}, b\right\rangle^{\prime}$ and $L_{8.1}^{+}=L_{8.2}^{+}=$ $\left\langle a^{2}, a b\right\rangle^{\prime}$. Let $\chi$ and $\psi$ be two primitive quartic Hecke characters associated with the cyclic quartic extensions $K_{8.1} / k_{2}$ and $L_{8.1} / k_{2}$, respectively. Then $\chi \psi^{2}$ and $\chi^{2} \psi$ are associated with the extensions $K_{8.2} / k_{2}$ and $L_{8.2} / k_{2}$, respectively. According to (17) in [Lou8] we have

$$
\begin{aligned}
h_{K_{8.1}}^{-} & =Q_{K_{8.1}} w_{K_{8.1}} N_{\mathbb{Q}(i) / \mathbb{Q}}\left(\frac{1}{2^{2}} L(0, \chi)\right), h_{K_{8.2}}^{-}=Q_{K_{8.2}} w_{K_{8.2}} N_{\mathbb{Q}(i) / \mathbb{Q}}\left(\frac{1}{2^{2}} L\left(0, \chi \psi^{2}\right)\right), \\
h_{L_{8.1}}^{-} & =Q_{L_{8.1}} w_{L_{8.1}} N_{\mathbb{Q}(i) / \mathbb{Q}}\left(\frac{1}{2^{2}} L(0, \psi)\right), h_{L_{8.2}}^{-}=Q_{L_{8.2}} w_{L_{8.2}} N_{\mathbb{Q}(i) / \mathbb{Q}}\left(\frac{1}{2^{2}} L\left(0, \chi^{2} \psi\right)\right), \\
h_{K_{8.1}}^{-} & =h_{L_{8.1}}^{-}, h_{K_{8.2}}^{-}=h_{L_{8.2}}^{-}
\end{aligned}
$$

and

$$
h_{N}^{-}=Q_{N} w_{N} N_{\mathbb{Q}(i) / \mathbb{Q}}\left(\frac{1}{2^{8}} L(0, \chi) L\left(0, \chi \psi^{2}\right) L(0, \psi) L\left(0, \chi^{2} \psi\right)\right),
$$

which proves $(\mathrm{G})$. Note that $w_{N}=w_{K_{8, i}}=w_{L_{8, i}}=2$ for $i=1,2$.

Part 3: Suppose that $h_{N}=1$. We claim the following:

(i) $h_{k_{2}}=h_{k_{3}}=1$. There exist two distinct primes $p, q \neq 3 \bmod 4$ such that $k_{1}=\mathbb{Q}(\sqrt{p q}), k_{2}=\mathbb{Q}(\sqrt{q})$, and $k_{3}=\mathbb{Q}(\sqrt{p})$. (More precisely, it will be verified in the proof of point (iv) below that $p=2$ or $p \equiv 1 \bmod 8$.) The ramification indices of $p$ and $q$ in $N / \mathbb{Q}$ are 4 and 2 , respectively.

(ii) Without loss of generality we may assume that $h_{K_{8.1}}=1, h_{K_{8.2}}^{-}=h_{K_{8.2}}=2$, and $Q_{K_{8.1}}=Q_{K_{8.2}}=1$. In addition, $p$ and $q$ are the only ramified primes in $N / \mathbb{Q}$.

(iii) Set $L_{4}=\left\langle a, b^{2}\right\rangle^{\prime}$. Then $L_{4}=\mathbb{Q}(\sqrt{p}, \sqrt{q}), h_{L_{4}}=2, \operatorname{cl}(\mathbb{Q}(\sqrt{p q}))=C_{4},\left(\frac{p}{q}\right)=$ $\left(\frac{q}{p}\right)=1$, and $N_{k_{1} / \mathbb{Q}}\left(\epsilon_{1}\right)=-1$, where $\epsilon_{1}$ is the fundamental unit of $k_{1}=\mathbb{Q}(\sqrt{p q})$.

(iv) Let $\mathfrak{p}$ and $\mathfrak{p}^{\prime}$ be the two prime ideals in $k_{2}$ lying above $p$. Then $\mathcal{F}\left(K_{8.1} / k_{2}\right)=$ $\mathfrak{p}$ and $\mathcal{F}\left(K_{8.2} / k_{2}\right)=\mathfrak{p p}^{\prime}$ if $p$ is odd, $\mathcal{F}\left(K_{8.1} / k_{2}\right)=\mathfrak{p}^{4}$ and $\mathcal{F}\left(K_{8.2} / k_{2}\right)=\mathfrak{p}^{4} \mathfrak{p}^{\prime 3}$ if $p=2$.

Before proving Part 3 we recall the following result.

Proposition 8 ( (Mas, Corollaries 2.2 and 2.3]). Let $E / F$ be an extension of number fields. Then $h_{F}$ divides $[E: F] h_{E}$. Moreover, if no non-trivial abelian subextension of $E / F$ is unramified over $F$, then $h_{F}$ divides $h_{E}$. 
Proof. (i) To begin with we will prove that $G\left(k_{2}\right)=k_{2}$ and $G\left(k_{3}\right)=k_{3}$. Note that there are five intermediate fields between $N$ and $k_{2}$ which are normal over $\mathbb{Q}$ : $\left\langle a^{2}\right\rangle^{\prime},\langle a\rangle^{\prime},\left\langle a^{2}, b^{2}\right\rangle^{\prime},\left\langle a b^{2}\right\rangle^{\prime}$, and $\left\langle a, b^{2}\right\rangle^{\prime}$. Moreover, $G_{32 / 31} /\left\langle a^{2}\right\rangle \simeq G_{6}$ in [JL (or type 16/9 in [TW]), $G_{32 / 31} /\langle a\rangle \simeq D_{8}, G_{32 / 31} /\left\langle a^{2}, b^{2}\right\rangle \simeq D_{8}, G_{32 / 31} /\left\langle a b^{2}\right\rangle \simeq C_{2} \times$ $C_{4}$, and $G_{32 / 31} /\left\langle a, b^{2}\right\rangle \simeq C_{2} \times C_{2}$. Since $h_{N}=1, G\left(k_{2}\right) \subset N$ and $G\left(k_{2}\right)=\left\langle a, b^{2}\right\rangle^{\prime}$ or $G\left(k_{2}\right)=k_{2}$. Since $\left\langle a, b^{2}\right\rangle^{\prime}$ contains $k_{1}, k_{2}$, and $k_{3}, G\left(k_{2}\right) \neq\left\langle a, b^{2}\right\rangle^{\prime}$. Otherwise, every prime divisor $p$ of $d_{k_{3}}$ divides $d_{k_{2}}$ and at the same time divides $d_{k_{1}}$ by Lemma 6. Thus, $p$ is totally ramified in $\left\langle a, b^{2}\right\rangle^{\prime} / \mathbb{Q}$. It is contrary to the fact that $\left\langle a, b^{2}\right\rangle^{\prime} / k_{2}$ is unramified. Hence, $k_{2}=G\left(k_{2}\right), h_{k_{2}}=1$, and $k_{2}=\mathbb{Q}(\sqrt{q})$ for some prime $q \neq \equiv$ $\bmod 4$. Similarly, $h_{k_{3}}=1$ and $k_{3}=\mathbb{Q}(\sqrt{p})$ for some prime $p \not \equiv 3 \bmod 4$.

(ii) Let $\mathfrak{P}$ be a prime ideal of $N$ dividing $p$ and $G_{0}(\mathfrak{P})$ its inertia group. Since $N / N^{+}$is unramified at all the finite places, $a^{2} \notin G_{0}(\mathfrak{P})$ and $G_{0}(\mathfrak{P})$ must be one of the four cyclic subgroups of order 4 of $G\left(N / k_{2}\right)=\langle a, b\rangle:\langle a b\rangle,\left\langle a b^{3}\right\rangle,\langle b\rangle,\left\langle a^{2} b\right\rangle$. Changing $\mathfrak{P}$ to $\mathfrak{P}^{\prime}=c \mathfrak{P}$, or $K_{8.1}$ to $K_{8.2}$ if necessary we may assume that $K_{8.1}=$ $G_{0}(\mathfrak{P})^{\prime}$. According to Proposition $8 h_{N_{16.1}}=h_{K_{8.1}}=1$, and $h_{K_{8.2}} \mid 2$, where $N_{16.1}=\left\langle b^{2}\right\rangle^{\prime}$. Then $G_{0}\left(\mathfrak{P}^{\prime}\right)=c G_{0}(\mathfrak{P}) c^{-1}=\left\langle a b^{3}\right\rangle$. Since $G\left(N / K_{8.1}^{+}\right)=\left\langle a^{2}, b\right\rangle$ and $G\left(N / K_{8.1}^{+}\right) \cap G_{0}\left(\mathfrak{P}^{\prime}\right)=\left\langle a^{2} b^{2}\right\rangle$, the prime ideal $\mathfrak{P}^{\prime} \cap O_{K_{8.2}}$ and the prime ideal $\mathfrak{P} \cap O_{K_{8.2}}$ are ramified in $K_{8.2} / K_{8.2}^{+}$. By Proposition $8 h_{K_{8.2}}^{-}=h_{K_{8.2}}=2$. From $(\mathrm{G})$ it follows that $Q_{K_{8.1}}=Q_{K_{8.2}}=1$. For the second assertion, we suppose that there exists a prime $r$ with $(r, p q)=1$ which is ramified in $N / \mathbb{Q}$. Its ramification index is equal to 2 . There are at least two prime ideals lying above $r$ in $N$. Let $\mathcal{R}$ be a prime ideal lying above $r$ such that its inertia group $G_{0}(\mathcal{R})$ is equal to $\left\langle a^{2} b^{2}\right\rangle$. Since $\left\langle a^{2} b^{2}\right\rangle \cap G\left(N / K_{8.1}\right)=\{1\}, \mathcal{R}$ is unramified in $N / K_{8.1}$. Since $G_{0}(\mathcal{R}) \subset G\left(N / K_{8.1}^{+}\right)$ and $G_{0}\left(\mathfrak{P}^{\prime}\right) \cap G\left(N / K_{8.1}^{+}\right)=\left\langle a^{2} b^{2}\right\rangle, \mathcal{R} \cap O_{K_{8.1}}$ and $\mathfrak{P}^{\prime} \cap O_{K_{8.1}}$ are ramified in $K_{8.1} / K_{8.1}^{+}$. It follows that 2 divides $h_{K_{8.1}}^{-}$, which contradicts $h_{K_{8.1}}=1$. Hence, $p$ and $q$ are the only prime divisors of $d_{N}$.

(iii) Since $G\left(N^{+} / k_{1}\right) \simeq\left\langle c b, b^{2}\right\rangle /\left\langle a^{2}\right\rangle \simeq C_{4} \times C_{2}$, there are three octic subfields of $N^{+}$containing $k_{1}$ : $\langle a\rangle^{\prime},\left\langle a^{2}, b^{2}\right\rangle^{\prime}$, and $\left\langle a b^{2}\right\rangle^{\prime}$. Since $N / N^{+}$is unramified at all the finite places, so are $N /\langle a\rangle^{\prime}$ and $N /\left\langle a b^{2}\right\rangle^{\prime}$. Let $B=\left\langle a^{2}, b^{2}\right\rangle^{\prime}$. Since $\left\langle a^{2}, b^{2}\right\rangle \triangleleft G$ and $\left\langle c b, b^{2}\right\rangle /\left\langle a^{2}, b^{2}\right\rangle \simeq C_{4}, G\left(B / k_{1}\right)$ is a cyclic group of order 4 . We claim that $B=\operatorname{Hil}\left(k_{1}\right)$. For every prime ideal lying above $p$ in $N^{+}$, its inertia group in the extension $N^{+} / k_{1}$ is of order 2 . Hence, it must be $G\left(N^{+} / B\right)$. Since the ramification index of $q$ in $N / \mathbb{Q}$ is equal to 2, the prime ideal lying above $q$ is unramified in $N^{+} / k_{1}$. By (ii) $p$ and $q$ are the only ramified primes in $N / \mathbb{Q}$. Hence, $B / k_{1}$ is unramified at all the places. From Proposition 8 it follows that $h_{B}=1, B=\operatorname{Hil}\left(k_{1}\right)$, and the unique intermediate field $L_{4}=\left\langle a, b^{2}\right\rangle^{\prime}=\mathbb{Q}(\sqrt{p}, \sqrt{q})$ between $B$ and $k_{1}$ has class number 2. According to [BLS, Proposition 1] $\left(\frac{q}{p}\right)=\left(\frac{p}{q}\right)=1$. Since $h_{N}=1$, $H i l\left(k_{1}\right)=H i l_{n a r}\left(k_{1}\right)$. Namely, $N_{k_{1} / \mathbb{Q}}\left(\epsilon_{1}\right)=-1$.

(iv) Using the discriminant-conductor formula for the abelian extension $N / k_{2}$ we can get the finite parts of the conductors $\mathcal{F}(\chi)\left(=\mathcal{F}\left(K_{8.1} / k_{2}\right)\right)$ and $\mathcal{F}\left(\chi \psi^{2}\right)(=$ $\left.\mathcal{F}\left(K_{8.2} / k_{2}\right)\right)$ from $d_{N}\left(=d_{L_{3}}^{8}\right)$. Let us determine $d_{L_{3}}$. If $p$ and $q$ are odd, then $d_{L_{3}}=$ $p^{3} q^{2}$. We claim that if $p$ is odd, then $p \equiv 1 \bmod 8$. Let $\left\langle a b^{2}\right\rangle^{\prime}=T$. Then $N / T$ is unramified at all the finite places. Since $\left\langle a b^{2}\right\rangle \triangleleft G_{32 / 31}$ and $G_{32 / 31} /\left\langle a b^{2}\right\rangle \simeq C_{4} \times C_{2}$, $T$ has two cyclic quartic subfields $L_{3}=\left\langle a b^{2}, a c\right\rangle^{\prime}$ and $L_{5}=\left\langle a b^{2}, c\right\rangle^{\prime}$. The inertia group of a prime ideal lying above $q$ in $T$ must be the Galois group $G\left(T / L_{5}\right)$. Hence $L_{5}$ is a totally real field of conductor $p$ if $p$ is odd, $2^{4}$ otherwise. Thus, $p \equiv 1 \bmod$ 8 if $p$ is odd. We have $d_{L_{3}}=2^{11} q^{2}$ if $p=2$ or $d_{L_{3}}=p^{3} 2^{6}$ if $q=2$. Note that the case that $d_{L_{3}}=p^{3} 2^{4}$ cannot occur. 
Part 4: There are 5447 pairs of $(p, q)$ which satisfy $d_{L_{3}}<8.7 \times 10^{14}, p=2$ or $p \equiv 1 \bmod 8, q \neq \equiv 3 \bmod 4, h_{\mathbb{Q}(\sqrt{p})}=h_{\mathbb{Q}(\sqrt{q})}=1,\left(\frac{q}{p}\right)=\left(\frac{p}{q}\right)=1, N_{k_{1} / \mathbb{Q}}\left(\epsilon_{1}\right)=-1$, and $h_{\mathbb{Q}(\sqrt{p q})}=4$. For these 5447 pairs of $(p, q)$ we compute explicitly $\kappa_{L_{4}}$. Using the better upper bound $\kappa_{N^{+}} \leq\left(\frac{\kappa_{L_{4}}}{6} \log \left(d_{N^{+}} / d_{L_{4}}^{4}\right)+\frac{\log ^{4} d_{L_{4}}}{2^{4} 3^{3}}\right)^{3} \kappa_{L_{4}}$ we improve the lower bound for $h_{N}^{-}$obtained in Part 1 as in the proof of Proposition 6. If $\zeta_{N}\left(\beta_{N}\right) \leq 0$, then

$$
h_{N}^{-} \geq \frac{d_{L_{3}}^{2}}{2^{16} e \pi^{16} \log d_{L_{3}}} \frac{1}{\left(\frac{\kappa_{L_{4}}}{6} \log \left(d_{N^{+}} / d_{L_{4}}^{4}\right)+\left(\log ^{4} d_{L_{4}}\right) /\left(2^{4} 3^{3}\right)\right)^{3} \kappa_{L_{4}}} .
$$

If $\zeta_{N}\left(\beta_{N}\right)>0$, then

$$
h_{N}^{-} \geq \frac{d_{L_{3}}^{2}}{2^{12} e \pi^{16} B_{N^{(2)}}} \frac{1}{\left(\frac{\kappa_{L_{4}}}{6} \log \left(d_{N^{+}} / d_{L_{4}}^{4}\right)+\left(\log ^{4} d_{L_{4}}\right) /\left(2^{4} 3^{3}\right)\right)^{3} \kappa_{L_{4}}} .
$$

For all of these 5447 pairs of $(p, q)$ we compute the lower bounds in (H) and (I) respectively, and then take the smaller one. There are 5236 out of these 5447 pairs with $h_{N}^{-}>1$. For the remaining $211(=5447-5236)$ pairs of $(p, q)$ we compute $h_{K_{8.1}}^{-}$and $h_{K_{8.2}}^{-}$. We examine whether there exist Hecke characters $\chi$ of order 4 with conductor $\mathfrak{p} \infty$ if $p \equiv 1 \bmod 8, \mathfrak{p}^{4} \infty$ otherwise. There are 103 pairs of $(p, q)$ that satisfy $\chi\left(\epsilon_{2}\right)=1$ and $\chi(-1)=1$, where $\epsilon_{2}$ denotes the fundamental unit of $k_{2}$. For these 103 pairs of $(p, q)$ we compute $L(0, \chi)$ by using the technique developed in Lou8 to obtain that $h_{K_{8.1}}^{-}=1$ if and only if $(p, q) \in\{(17,53),(89,5),(137,2)\}$. For these three fields we compute $h_{K_{8.2}}^{-}$and verify that there is no pair of $\left(K_{8.1}, K_{8.2}\right)$ such that $\left(h_{K_{8.1}}^{-}, h_{K_{8.2}}^{-}\right)=(1,2)$. Our computational results are given in the table below.

\begin{tabular}{|r|c|c|c|c|c|}
\hline$(p, q)$ & $L(0, \chi)$ & $h_{K_{8,1}}^{-}$ & $L\left(0, \chi \psi^{2}\right)$ & $h_{K_{8,2}}^{-}$ & $h_{N}^{-}$ \\
\hline$(17,53)$ & $2+2 i$ & 1 & $-8-4 i$ & 10 & $5^{2}$ \\
\hline$(89,5)$ & $2+2 i$ & 1 & $-24-4 i$ & 74 & $37^{2}$ \\
\hline$(137,2)$ & $-2-2 i$ & 1 & $-8+36 i$ & 170 & $85^{2}$ \\
\hline
\end{tabular}

It follows that there is no field $N$ with $h_{N}=1$, as claimed.

Now, the proof of point (1) of Theorem 1 is complete.

\section{The Composita $N=M_{16.1} M_{16.2}$}

The aim of this section is to prove point (2) of Theorem 1. In this section $N=M_{16.1} M_{16.2}$ is a normal CM-field of degree 32 which is a compositum of two normal CM-fields, $M_{16.1}$ and $M_{16.2}$, of degree 16 over $\mathbb{Q}$ with the same maximal real octic subfield $M_{8}^{+}:=M_{16.1}^{+}=M_{16.2}^{+}$. In order to determine all such fields $N$ with $h_{N}^{-}=1$ we divide our computations into 4 cases according to the Galois group $G\left(M_{8}^{+} / \mathbb{Q}\right) \in\left\{D_{8}, Q_{8}, C_{4} \times C_{2}, C_{2} \times C_{2} \times C_{2}\right\}$. It is known that if $L$ is an imaginary abelian number field with relative class number one, then $[L: \mathbb{Q}] \leq 24$ ([CK]). If $h_{N}^{-}=1$, then $G\left(M_{8}^{+} / \mathbb{Q}\right)$ cannot be isomorphic to $C_{8}$. Otherwise, $M_{16.1}, M_{16.2}$, and $N$ are imaginary abelian number fields and $h_{N}^{-}>1$. For an abelian number field $K$ we write $G(K / \mathbb{Q})=C_{m}^{*} \times C_{n}$ when $K=L k$ is a compositum of an imaginary cyclic number field $L$ of degree $m$ over $\mathbb{Q}$ and a real cyclic number field $k$ of degree $n$ with $L \cap k=\mathbb{Q}$. 
Proposition 9 ([J] and [TW]). There are 9 non-abelian groups of order 16 :

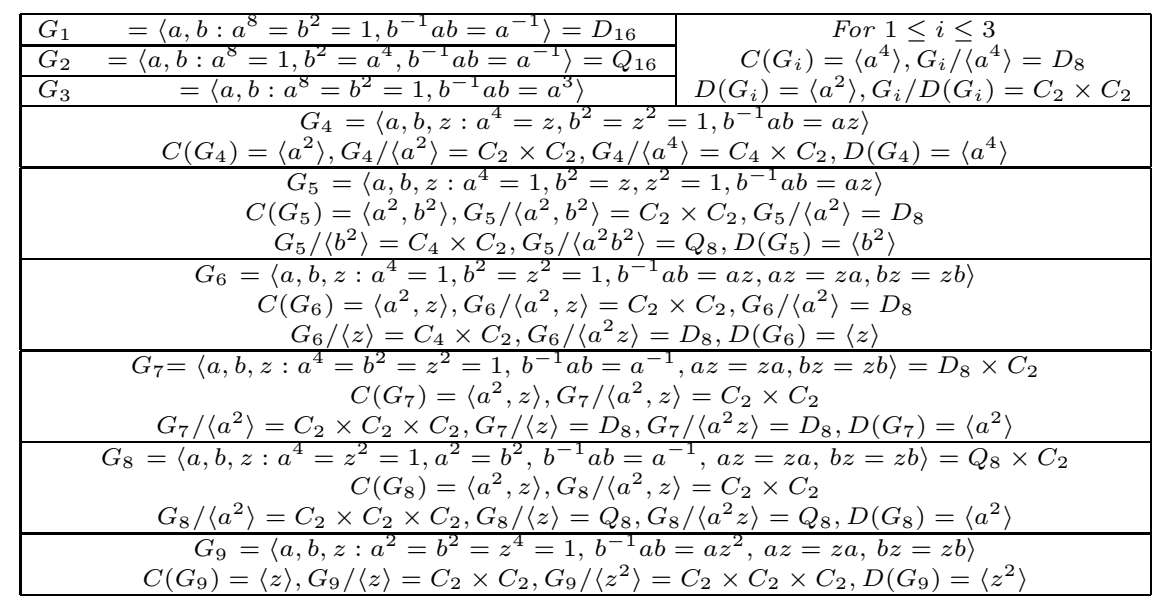

Theorem 3 ([LO2 and Lou3]). There are precisely 13 non-abelian normal $C M$ fields $M$ of degree 16 with relative class number one: five with $G(M / \mathbb{Q})=D_{16}$, one with $G(M / \mathbb{Q})=G_{6}$ and $G\left(M^{+} / \mathbb{Q}\right)=C_{4} \times C_{2}$, one with $G(M / Q)=G_{6}$ and $G\left(M^{+} / \mathbb{Q}\right)=D_{8}$, one with $G(M / \mathbb{Q})=G_{7}$ and $G\left(M^{+} / Q\right)=C_{2} \times C_{2} \times C_{2}$, three with $G(M / \mathbb{Q})=G_{7}$ and $G\left(M^{+} / \mathbb{Q}\right)=D_{8}$, one with $G(M / \mathbb{Q})=G_{8}$ and $G\left(M^{+} / \mathbb{Q}\right)=Q_{8}$, and one with $G(M / \mathbb{Q})=G_{9}$.

Proof. In [LO2] and [Lou3] the fields with relative class number one are known except for the fields which are composita of an abelian octic CM-field and a dihedral octic CM-field. There are four non-abelian CM-fields of degree 16 with class number one which are composita of an abelian octic CM-field and a dihedral octic CM-field with the same maximal totally real subfield ([Lou3). Using CK and YK we verify that those are the only such fields with relative class number one.

Proposition 10. Let $N=M_{16.1} M_{16.2}$ be a compositum of two normal $C M$-fields of degree 16 with the same totally real octic subfield $M_{16.1}^{+}=M_{16.2}^{+}$. Assume that $M_{16.1}=M_{8.1} M_{8.2}$ is a compositum of two isomorphic octic CM-fields with the same totally real quartic subfield $M_{8.1}^{+}=M_{8.2}^{+}$. If $h_{N}^{-}=1$ and $w_{M_{16.1}}=2$, then $h_{M_{16.1}}^{-}=1$ or $h_{M_{16.2}}^{-}=1$. In particular, if $h_{N}^{-}=1$ and $G\left(M_{16.1} / \mathbb{Q}\right) \in\left\{G_{1}, G_{9}\right\}$, then $h_{M_{16.1}}^{-}=1$ or $h_{M_{16.2}}^{-}=1$, if $h_{N}^{-}=1$ and $G\left(M_{16.1} / \mathbb{Q}\right) \in\left\{G_{3}, G_{4}\right\}$, then $h_{M_{16.1}}^{-}=2 Q_{M_{16.1}}$ and $h_{M_{16.2}}^{-}=1$.

Proof. By Theorem 2 (1) we have

$$
h_{N}^{-}=\frac{Q_{N}}{Q_{M_{16.2}}} \frac{w_{N}}{w_{M_{16.2}}} h_{M_{16.2}}^{-} \frac{h_{M_{16.1}}^{-}}{Q_{M_{16.1}} w_{M_{16.1}}}
$$

and

$$
h_{M_{16.1}}^{-}=\frac{Q_{M_{16.1}}}{2}\left(\frac{h_{M_{8.1}}^{-}}{Q_{M_{8.1}}}\right)^{2} .
$$

By Theorem 2 (2) it follows that $Q_{N} w_{N} /\left(Q_{M_{16.2}} w_{M_{16.2}}\right)$ is an integer. If $h_{N}^{-}=1$ and $h_{M_{16.1}}^{-} \neq 1$, then $h_{M_{16.1}}^{-} / Q_{M_{16.1}}=2$. Hence, $h_{M_{16.1}}^{-} /\left(Q_{M_{16.1}} w_{M_{16.1}}\right)=1$ and $h_{M_{16.2}}^{-}=1$. Now, we claim that if $G\left(M_{16.1} / \mathbb{Q}\right) \in\left\{G_{1}, G_{3}, G_{4}, G_{9}\right\}$, then $G\left(M_{16.1} / \mathbb{Q}\right)$ has two elements $\alpha$ and $\beta$ of order 2 such that $\beta=\gamma \alpha \gamma^{-1}$ for some $\gamma \in G\left(M_{16.1} / \mathbb{Q}\right),\langle\alpha, \beta\rangle$ 
TABLe 1 . Here $k_{2}$ is the unique quadratic real subfield of $M$ such that $G\left(M^{+} / k_{2}\right)=C_{4}$.

\begin{tabular}{|ccccccc|}
\hline$N r$. & $M$ & $M^{+}$ & $k_{2}$ & $G(M / \mathbb{Q})$ & $Q_{M}$ & $h_{M}$ \\
1 & $H i l^{+}(\mathbb{Q}(\sqrt{514}))$ & $\mathbb{Q}(\sqrt{257}, \sqrt{17+4 \sqrt{2}})$ & $\mathbb{Q}(\sqrt{514})$ & $D_{16}$ & 2 & 3 \\
2 & $H i l^{+}(\mathbb{Q}(\sqrt{505}))$ & $\mathbb{Q}(\sqrt{101}, \sqrt{11+2 \sqrt{5}})$ & $\mathbb{Q}(\sqrt{505})$ & $D_{16}$ & 2 & 1 \\
3 & $H i l^{+}(\mathbb{Q}(\sqrt{905}))$ & $\mathbb{Q}(\sqrt{181}, \sqrt{(27+\sqrt{5}) / 2})$ & $\mathbb{Q}(\sqrt{905})$ & $D_{16}$ & 2 & 1 \\
4 & $H i l^{+}(\mathbb{Q}(\sqrt{689}))$ & $\mathbb{Q}(\sqrt{53}, \sqrt{(15+\sqrt{13}) / 2})$ & $\mathbb{Q}(\sqrt{689})$ & $D_{16}$ & 2 & 1 \\
5 & $H i l^{+}(\mathbb{Q}(\sqrt{793}))$ & $\mathbb{Q}(\sqrt{61}, \sqrt{(19+3 \sqrt{13}) / 2})$ & $\mathbb{Q}(\sqrt{793})$ & $D_{16}$ & 2 & 1 \\
6 & $M^{+}(\sqrt{-1})$ & $\mathbb{Q}(\sqrt{2}, \sqrt{3+\sqrt{3}})$ & $\mathbb{Q}(\sqrt{2})$ & $G_{7}$ & 2 & 1 \\
7 & $M^{+}(\sqrt{-1})$ & $\mathbb{Q}(\sqrt{11}, \sqrt{15+8 \sqrt{3}})$ & $\mathbb{Q}(\sqrt{11})$ & $G_{7}$ & 2 & 1 \\
8 & $M^{+}(\sqrt{-3})$ & $\mathbb{Q}(\sqrt{2}, \sqrt{(15+3 \sqrt{17}) / 2})$ & $\mathbb{Q}(\sqrt{34})$ & $G_{7}$ & 2 & 1 \\
9 & $\left.M^{+}(\sqrt{-(13+2 \sqrt{13}})\right)$ & $\mathbb{Q}(\sqrt{17}, \sqrt{(143+31 \sqrt{13}}) / 2$ & $\mathbb{Q}(\sqrt{17})$ & $G_{6}$ & 2 & 1 \\
\hline
\end{tabular}

contains the complex conjugation $\tau$, and $D\left(G\left(M_{16.1} / \mathbb{Q}\right)\right)$ contains $\tau$. The fact that $\tau \in D\left(G\left(M_{16.1} / \mathbb{Q}\right)\right)$ ensures that $w_{M_{16.1}}=2$. It is sufficient to take $\alpha$ and $\beta$ as follows (with the notation of Proposition 9):

\begin{tabular}{|c|c|c|c|c|}
\hline & $G_{1}$ & $G_{3}$ & $G_{4}$ & $G_{9}$ \\
\hline$\alpha$ & $b$ & $b$ & $b$ & $a$ \\
\hline$\beta$ & $a^{-2} b a^{2}=a^{4} b$ & $a^{-2} b a^{2}=a^{4} b$ & $a^{-1} b a=a^{4} b$ & $b^{-1} a b=a z^{2}$ \\
\hline$\tau$ & $a^{4}$ & $a^{4}$ & $a^{4}$ & $z^{2}$ \\
\hline
\end{tabular}

4.1. In this subsection we assume that $G\left(M_{8}^{+} / \mathbb{Q}\right)=D_{8}$. Then we have $G\left(M_{16.1} / \mathbb{Q}\right)$, $G\left(M_{16.2} / \mathbb{Q}\right) \in\left\{D_{16}, Q_{16}, G_{3}, G_{5}, G_{6}, G_{7}\right\}$. In Table 1 we list the nine normal CMfields $M$ of degree 16 such that $h_{M}^{-}=1$ and $G\left(M^{+} / \mathbb{Q}\right)=D_{8}$ (see $[\mathrm{LO} 2$, Theorem 10] and [Lou3, Theorem 3]). Using [KT] we verify the following which will be used in the proofs of several propositions.

Lemma 7. (1) If $k=\mathbb{Q}(\sqrt{2}, \sqrt{3+\sqrt{3}}), 2 O_{k}=\mathcal{I}^{8}$ and $3 O_{k}=\mathcal{J}^{4}$, then there are exactly four totally imaginary quadratic extensions $K$ of $k$ such that $K$ is a subfield of the ray class field of $k$ with modulus $\mathcal{I}^{17} \mathcal{J} \infty$ and $K$ is normal over $\mathbb{Q}: H_{i l_{\text {nar }}}(k)$ with Galois group $G_{7}$, one of conductor $\mathcal{I}^{10} \mathcal{J} \infty$ with Galois group $G_{5}$, and two of conductor $\mathcal{I}^{14} \mathcal{J} \infty$ with Galois group $G_{3}$.

(2) If $k=\mathbb{Q}(\sqrt{11}, \sqrt{15+8 \sqrt{3}})$ and $3 O_{k}=\mathcal{I}^{4}$, then the ray class field of $k$ with modulus $\mathcal{I} \infty$ contains one and only one quadratic extension $K$ of $k$ which is normal over $\mathbb{Q}: H_{i l_{n a r}}(k)$ with Galois group $G_{7}$.

(3) If $k=\mathbb{Q}(\sqrt{2}, \sqrt{(15+3 \sqrt{17}) / 2})$ and $3 O_{k}=\mathcal{J}_{1} \mathcal{J}_{2}$, then there is no quadratic extension of $k$ with conductor $\mathcal{J}_{1} \mathcal{J}_{2} \infty$.

(4) If $k=\mathbb{Q}(\sqrt{2}, \sqrt{5}, \sqrt{17}), 2 O_{k}=\mathcal{I}_{2}^{2} \mathcal{I}_{2}^{\prime 2}, 5 O_{k}=\mathcal{I}_{5}^{2} \mathcal{I}_{5}^{\prime 2}$ and $17 O_{k}=\mathcal{I}_{17}^{2} \mathcal{I}_{17}^{\prime 2}$, then there is no totally imaginary quadratic extension $K$ of $k$ such that $\mathcal{F}(K / k) \in\left\{\mathcal{I}_{2}^{e} \mathcal{I}_{2}^{\prime e}, \mathcal{I}_{5} \mathcal{I}_{5}^{\prime}, \mathcal{I}_{17} \mathcal{I}_{17}^{\prime}\right\}$ and $K / \mathbb{Q}$ is normal with $G(K / \mathbb{Q})=G_{9}$, where $e=2,4$, or 5 .

(5) If $k=\mathbb{Q}(\sqrt{2}, \sqrt{5}, \sqrt{37}), 2 O_{k}=\mathcal{I}_{2}^{2} \mathcal{I}_{2}^{\prime 2}, 5 O_{k}=\mathcal{I}_{5}^{2} \mathcal{I}_{5}^{\prime 2}$ and $37 O_{k}=\mathcal{I}_{37}^{2} \mathcal{I}_{37}^{\prime 2}$, then there is no totally imaginary quadratic extension $K$ of $k$ such that $\mathcal{F}(K / k) \in\left\{\mathcal{I}_{2}^{e} \mathcal{I}_{2}^{\prime e}, \mathcal{I}_{5} \mathcal{I}_{5}^{\prime}, \mathcal{I}_{37} \mathcal{I}_{37}^{\prime}\right\}$ and $K / \mathbb{Q}$ is normal with $G(K / \mathbb{Q})=G_{9}$, where $e=2$, 4 , or 5 . 
(6) If $k=\mathbb{Q}(\sqrt{17+4 \sqrt{17}}), 2 O_{k}=\mathfrak{p}_{2} \mathfrak{p}_{2}^{\prime}$ and $17 O_{k}=\mathfrak{p}_{17}^{4}$, then there is one and only one (up to isomorphism) quadratic extension $K$ of $k$ such that the conductor of $K / k$ is of the form $\mathfrak{p}_{2}^{e} \mathfrak{p}_{17} \infty$ with $e=2$ or 3 and the normal closure $N$ of $K$ has $G(N / \mathbb{Q})=G_{4}: \mathcal{F}(K / k)=\mathfrak{p}_{2}^{3} \mathfrak{p}_{17}$ and $K=\mathbb{Q}(\theta)$ with $\theta^{8}+17 \theta^{6}+85 \theta^{4}+136 \theta^{2}+68=0$.

(7) If $k=\mathbb{Q}(\sqrt{5}, \sqrt{(65+\sqrt{65}) / 2}), 5 O_{k}=\mathfrak{P}_{5}^{4}$ and $13 O_{k}=\mathfrak{P}_{13}^{4}$, then there is one and only one normal $C M$-field $K$ containing $k$ with $G(K / \mathbb{Q})=G_{4}$ and $\mathcal{F}(K / k)=\mathfrak{P}_{5} \mathfrak{P}_{13}$.

(8) If $k=\mathbb{Q}(\sqrt{(5-\sqrt{5}) / 2}), 2 O_{k}=\mathfrak{p}_{2}^{2}$ and $5 O_{k}=\mathfrak{p}_{5}^{4}$, then there is one and only one (up to isomorphism) quadratic extension $K$ of $k$ such that the conductor of $K / k$ is of the form $\mathfrak{p}_{2}^{e} \mathfrak{p}_{5} \infty$ with $e \in\{2,4,5\}$ and the normal closure $N$ of $K$ satisfies $G(N / \mathbb{Q})=G_{4}: \mathcal{F}(K / k)=\mathfrak{p}_{2}^{4} \mathfrak{p}_{5}$ and $K=\mathbb{Q}(\theta)$ with $\theta^{8}+10 \theta^{6}+25 \theta^{4}+20 \theta^{2}+5=0$.

(9) If $k=\mathbb{Q}(\sqrt{5+\sqrt{5}}), 2 O_{k}=\mathfrak{p}_{2}^{2}$ and $5 O_{k}=\mathfrak{p}_{5}^{4}$, then there is no quadratic extension $K$ of $k$ such that the conductor of $K / k$ is of the form $\mathfrak{p}_{2}^{e} \mathfrak{p}_{5} \infty$ with $e \in\{2,4,5\}$ and the normal closure $N$ of $K$ satisfies $G(N / \mathbb{Q})=G_{4}$.

(10) If $k=\mathbb{Q}(\sqrt{2+\sqrt{2}})$ and $2 O_{k}=\mathfrak{p}_{2}^{4}$, then there is no quadratic extension $K$ of $k$ such that the conductor of $K / k$ is of the form $\mathfrak{p}_{2}^{e}(3)^{g} \infty$ and the normal closure $N$ of $K$ satisfies $G(N / \mathbb{Q})=G_{4}$, where $e \in\{2,4,6,8,9\}$ and $g \in\{0,1\}$.

(11) If $k=\mathbb{Q}(\sqrt{3(2+\sqrt{2})}), 2 O_{k}=\mathfrak{p}_{2}^{4}$ and $3 O_{k}=\mathfrak{p}_{3}^{2}$, then there is one and only one (up to isomorphism) quadratic extension $K$ of $k$ such that the conductor of $K / k$ is of the form $\mathfrak{p}_{2}^{e} \mathfrak{p}_{3} \infty$ and the normal closure $N$ of $K$ satisfies $G(N / \mathbb{Q})=G_{4}$, where $e \in\{2,4,6,8,9\}$ : this extension $K / k$ is of conductor $\mathfrak{p}_{2}^{9} \mathfrak{p}_{3} \infty$.

Proposition 11. If any one of $G\left(M_{16 . i} / \mathbb{Q}\right)$ for $i=1,2$ is isomorphic to $G_{2}$ or $G_{3}$, then $h_{N}^{-}>1$.

Proof. (i) Assume that $G\left(M_{16.1} / \mathbb{Q}\right)=G_{2}\left(=Q_{16}\right)$. According to [Lou6, Theorem 3] $2 \mid h_{M_{16.1}}^{-}$and $Q_{M_{16.1}}=1$. By $(\mathrm{J})$ if $h_{N}^{-}=1$, then $h_{M_{16.1}}^{-}=2, h_{M_{16.2}}^{-}=$ 1, and $M_{16.2}$ must be one of the nine fields $M$ in Table 1 . Hence, we have $M_{16.2}=\mathbb{Q}(\sqrt{-1}, \sqrt{2}, \sqrt{3+\sqrt{3}})$. Indeed, for the eight other $M$ 's in Table 1 at least four prime ideals are ramified in $M^{+} / \mathbb{Q}$ (here $M^{+}=M_{16.1}^{+}=M_{16.2}^{+}$), and since $G\left(M_{16.1} / \mathbb{Q}\right)=Q_{16}$, these four prime ideals of $M_{16.1}^{+}$are ramified in $M_{16.1} / M_{16.1}^{+}$and $2^{3} \mid h_{M_{16.1}}^{-}$. Now, $(2)=\mathcal{I}^{8}$ and $(3)=\mathcal{J}^{4}$ in $M_{16.1}^{+}=\mathbb{Q}(\sqrt{2}, \sqrt{3+\sqrt{3}})$. We need to verify whether there exists a normal CM-field $M_{16.1}$ containing $\mathbb{Q}(\sqrt{2}, \sqrt{3+\sqrt{3}})$ such that $G\left(M_{16.1} / \mathbb{Q}\right)=Q_{16}$ and $h_{M_{16.1}}^{-}=2$. Since $\mathbb{Q}(\sqrt{2}, \sqrt{3+\sqrt{3}}) / \mathbb{Q}(\sqrt{2})$ is cyclic, $\mathcal{F}\left(M_{16.1} / M_{16.1}^{+}\right)=\mathcal{I}^{e} \mathcal{J}$ for some $2 \leq e \leq 17$. (See [He, Theorem 119] and Co, Theorem 10.2.9].) According to Lemma 7, point (1), there is no such field $M_{16.1}$. We conclude that if any one of $G\left(M_{16 . i} / \mathbb{Q}\right)$ for $i=1,2$ is isomorphic to $G_{2}$, then $h_{N}^{-}>1$.

(ii) Assume that $G\left(M_{16.1} / \mathbb{Q}\right)=G_{3}$. By Proposition 10 if $h_{N}^{-}=1$, then $h_{M_{16.1}}^{-}=$ $2 Q_{M_{16.1}}, h_{M_{16.2}}^{-}=1$ and $M_{16.2}$ must be one of the nine fields in Table 1 . For a given octic dihedral field $M^{+}$in Table 1 we will construct $M_{16.1}$ containing $M^{+}$such that $G\left(M_{16.1} / \mathbb{Q}\right)=G_{3}$. Note that the subgroup $\left\langle a^{2}, a b\right\rangle=Q_{8}$ and $\left\langle a^{2}, b\right\rangle=D_{8}$. 
(ii-a) Suppose that $M_{16.1} \supset \mathbb{Q}(\sqrt{257}, \sqrt{17+4 \sqrt{2}})$. Then $\left\langle a^{2}, a b\right\rangle^{\prime}=\mathbb{Q}(\sqrt{257})$ or $\mathbb{Q}(\sqrt{2})$. If $G\left(M_{16.1} / \mathbb{Q}(\sqrt{2})\right)=Q_{8}$, then the four prime ideals lying above 257 are ramified in the quadratic extension $M_{16.1} / \mathbb{Q}(\sqrt{257}, \sqrt{17+4 \sqrt{2}})$, whence $2^{3} \mid h_{M_{16.1}}^{-}$. If $G\left(M_{16.1} / \mathbb{Q}(\sqrt{257})\right)=Q_{8}$, then the four prime ideals lying above 2 are ramified in the quadratic extension $M_{16.1} / \mathbb{Q}(\sqrt{257}, \sqrt{17+4 \sqrt{2}})$, whence $2^{3} \mid h_{M_{16.1}^{-}}$. By the same argument we verify that if there exists a normal CM-field $M_{16.1}$ such that $G\left(M_{16.1} / \mathbb{Q}\right)=G_{3}$ and $M_{16.1}^{+}$is one of eight fields $M^{+}$in Table 1 except for the field numbered 6 , then $4 \mid h_{M_{161}}^{-}$.

(ii-b) We take the field number 6 in Table 1 as $M_{16.2}$ and assume that $G\left(M_{16.1} / \mathbb{Q}\right)$ $=G_{3}$ and $M_{16.1}^{+}=\mathbb{Q}(\sqrt{2}, \sqrt{3+\sqrt{3}})$. Since $h_{M_{16.1}}^{-}=2 Q_{M_{16.1}}$, at most two prime ideals are ramified in $M_{16.1} / M_{16.1}^{+}$and $\mathcal{F}\left(M_{16.1} / M_{16.1}^{+}\right)=\mathcal{I}^{e} \mathcal{J}$ for some $2 \leq e \leq 17$, where $\mathcal{I}$ and $\mathcal{J}$ are as in (i). From Lemma 7, point (1), there are two such fields $M_{16.1}$ and $M_{16.1}^{\prime}: \mathcal{F}\left(M_{16.1} / \mathbb{Q}(\sqrt{2}, \sqrt{3+\sqrt{3}})\right)=\mathcal{F}\left(M_{16.1}^{\prime} / \mathbb{Q}(\sqrt{2}, \sqrt{3+\sqrt{3}})\right)=$ $\mathcal{I}^{14} \mathcal{J}, G\left(M_{16.1} / \mathbb{Q}(\sqrt{3})\right)=D_{8}, G\left(M_{16.1} / \mathbb{Q}(\sqrt{6})\right)=Q_{8}, G\left(M_{16.1}^{\prime} / \mathbb{Q}(\sqrt{6})\right)=D_{8}$, and $G\left(M_{16.1}^{\prime} / \mathbb{Q}(\sqrt{3})\right)=Q_{8}$. To determine $h_{M_{16.1}}^{-}$and $h_{M_{16.1}^{\prime}}^{-}$we compute the relative class numbers of their non-normal octic CM-subfields by using the technique developed in [Lou7]: the quadratic extension $M_{8.1}$ of $\mathbb{Q}(\sqrt{3+\sqrt{3}})\left(=M_{8.1}^{+}\right)$with conductor $\mathfrak{p}_{3} \mathfrak{p}_{2}^{9} \infty$ for $h_{M_{16.1}}^{-}$and the quadratic extension $M_{8.1}^{\prime}$ of $\mathbb{Q}(\sqrt{3+\sqrt{6}})\left(=M_{8.1}^{\prime+}\right)$ with conductor $\mathfrak{p}_{3}^{\prime} \mathfrak{p}_{2}^{\prime 8} \infty$ for $h_{M_{16.1}^{\prime}}^{-}$, where $\mathfrak{p}_{3}, \mathfrak{p}_{3}^{\prime}$ are the unique prime ideal lying above 3 in $M_{8.1}^{+}$and $M_{8.1}^{\prime+}$ respectively, and $\mathfrak{p}_{2}, \mathfrak{p}_{2}^{\prime}$ are those above 2. We obtain $h_{M_{8.1}}^{-}=h_{M_{8.1}^{\prime}}^{-}=6$. By point (2) of Theorem 2 it follows that neither $h_{M_{16.1}}^{-}$nor $h_{M_{16.1}^{\prime}}^{-}$divides 4 , and $h_{N}^{-}>1$.

In the following Propositions $12-15$ we prove that if any one of $G\left(M_{16 . i} / \mathbb{Q}\right)$ for $i=1,2$ is isomorphic to $D_{16}$, then $h_{N}^{-} \geq 2$.

Lemma 8. Let $N=M_{16.1} M_{16.2}$ be a compositum of two dihedral CM-fields $M_{16.1}$ and $M_{16.2}$ of degree 16 with $M_{16.1}^{+}=M_{16.2}^{+}$. Assume that $h_{M_{16.1}}^{-}=1$. Then $h_{N}^{-}=1$ if and only if $h_{M_{16.2}}^{-}=2$.

Proof. Since there is no pair of $\left(M_{16.1}, M_{16.2}\right)$ such that $h_{M_{16.1}}^{-}=h_{M_{16.2}}^{-}=1$ and $M_{16.1}^{+}=M_{16.2}^{+}$(see [Lef] and [Lou6]), it is sufficient to show that $Q_{M_{16.2}}$ must be equal to 1 in order to prove that $h_{N}^{-}=1$ implies $h_{M_{16.2}}^{-}=2$. According to $\mathrm{LO} 2$, Theorem10] $M_{16.1}$ must be one of the fields numbered from 1 to 5 in Table 1. For these five fields we have $Q_{M_{16.1}}=2, h_{M_{16.1}^{+}}$is odd, and $h_{M_{16.1}^{+}}^{+}=2 h_{M_{16.1}^{+}} \equiv 2 \bmod$ 4 , where $h_{M_{16.1}^{+}}^{+}$is the narrow class number of $M_{16.1}^{+}$. Hence $M_{16.1}=M_{16.1}^{+}(\sqrt{-\epsilon})$, where $\epsilon \neq 1$ is a totally positive unit of $M_{16.1}^{+}$. There is only one such unit up to square. Suppose that $Q_{M_{16.2}}=2$. By Theorem 2 (4) we would have $M_{16.2}=$ $M_{16.2}^{+}(\sqrt{-\epsilon})=M_{16.1}$. This is a contradiction. Now, assume that $h_{M_{16.2}}^{-}=2$. It follows that $w_{N}=Q_{N}=2$ and $h_{N}^{-}=1$.

Proposition 12. If $N=M_{16.1} M_{16.2}$ is a compositum of two dihedral CM-fields of degree 16 with $M_{16.1}^{+}=M_{16.2}^{+}$, then $h_{N}^{-} \geq 2$.

Proof. By Proposition 10 and Lemma 8 it is sufficient to show that there is no pair of $\left(M_{16.1}, M_{16.2}\right)$ such that $h_{M_{16.1}}^{-}=1$ and $h_{M_{16.2}}^{-}=2$. To do this we will prove that there is no dihedral CM-field $M_{16}$ of degree 16 with $h_{M_{16}}^{-}=2$ such that $M_{16}^{+}$is 
equal to any one of the five octic subfields $M^{+}$of $M$ numbered from 1 to 5 in Table 1. We proceed as follows. First, we fix the real octic field $M^{+}$and find a constant $C_{0}$ such that if $h_{M_{16}}^{-} \leq 2$ and $M_{16}^{+}=M^{+}$, then $d_{M_{16}} \leq C_{0}$. Second, we make a list of dihedral CM-fields $M_{16}$ such that $M_{16}^{+}=M^{+}, d_{M_{16}} \leq C_{0}$, and exactly two prime ideals are ramified in $M_{16} / M_{16}^{+}$. Third, we compute the relative class numbers of these dihedral fields $M_{16}$.

(i) Let $M_{4}$ be the only quartic normal subfield of $M_{16}^{+}$and $k_{2}$ as in Table 1 . We have

$$
\left(\zeta_{M_{16}} / \zeta_{M_{4}}\right)(s)=\prod_{i=1}^{3}\left|L\left(s, \chi^{i}, M_{16} / k_{2}\right)\right|^{2} \geq 0
$$

where $\chi$ is any one of four octic characters of degree one associated with the cyclic extension $M_{16} / k_{2}$. For all five subfields $M_{4}$ in $M^{+}$in Table 1 we verify that $\zeta_{M_{4}}(s) \leq$ 0 for $s \in(0,1)$, whence $\zeta_{M_{16}}(s) \leq 0$ for $s \in(0,1)$. Now, we apply Theorem 2, points (7) and (8), and get

$$
\kappa_{M_{16}} \geq 2 /\left(e \log d_{M_{16}}\right)
$$

and

$$
\kappa_{M_{16}^{+}} \leq \kappa_{k_{2}}\left(\mu_{k_{2}} \kappa_{k_{2}}\right)^{3} \leq \frac{\log ^{7} d_{k_{2}}}{2^{10}} .
$$

Let $\mathfrak{D}$ be the relative discriminant of the extension $M_{16} / M_{16}^{+}$. Using $(\mathrm{K})$ and $(\mathrm{L})$ we obtain in Table 2 an upper bound $C$ such that $N_{M_{16}^{+} / \mathbb{Q}}(\mathfrak{D})^{1 / 8} \leq C$ if $h_{M_{16}}^{-} \leq 2$.

(ii) The fact that $h_{M_{16}}^{-}=2$ implies that $N_{M_{16}^{+} / \mathbb{Q}}\left(\mathcal{F}\left(M_{16} / M_{16}^{+}\right)\right)$has only one prime divisor $p$, and this $p$ is not ramified in $M_{16}^{+}: p O_{M_{16}^{+}}=\mathfrak{p p}^{\prime}, p O_{M_{16}}=\mathfrak{P}^{2} \mathfrak{P}^{2}$, where $\mathfrak{P}$ and $\mathfrak{P}^{\prime}$ are the unique ramified prime ideals in $M_{16} / M_{16}^{+}$. Indeed, the primes ramified in $k_{2}$ split completely in $M_{16}^{+}=H i l\left(k_{2}\right)$. Hence, if $h_{M_{16}}^{-}=2$, then the prime divisors of $d_{M_{16}^{+}}$cannot be ramified in $M_{16} / M_{16}^{+}$. Let $L$ be any one of two quadratic subfields of $M_{16}^{+}$such that $G\left(M_{16}^{+} / L\right)=C_{2} \times C_{2}$. Then $G\left(M_{16} / L\right)=D_{8}$. Let $K_{4}$ be any one of the two non-normal quartic subfields of $M_{16}^{+}$containing $L$ and let $K_{8}$ be any one of the two non-normal octic CMsubfields of $M_{16}$ containing $K_{4}$. (Hence $K_{4}=K_{8}^{+}$.) Let us determine $\mathcal{F}\left(K_{8} / K_{4}\right)$. In a normal extension $K / k$ for a prime ideal $\mathfrak{Q}$ of $K$ we denote by $G_{-1}(\mathfrak{Q})$ and $G_{0}(\mathfrak{Q})$ the decomposition group and the inertia group of $\mathfrak{Q}$, respectively. Since $G_{-1}(\mathfrak{p})=G_{-1}\left(\mathfrak{p}^{\prime}\right)=G\left(M_{16}^{+} / k_{2}\right)$ and $G_{0}(\mathfrak{p})=G_{0}\left(\mathfrak{p}^{\prime}\right)=\{i d\}, p O_{K_{4}}$ remains prime. Since $G_{-1}(\mathfrak{P})=G_{-1}\left(\mathfrak{P}^{\prime}\right)=G\left(M_{16} / k_{2}\right)$ and $G_{0}(\mathfrak{P})=G_{0}\left(\mathfrak{P}^{\prime}\right)=G\left(M_{16} / M_{16}^{+}\right)$, $p O_{K_{4}}$ is ramified in $K_{8} / K_{4}$. Let us write $k_{2}=\mathbb{Q}(\sqrt{r s})$ with $r, s$ two distinct primes and $L=\mathbb{Q}(\sqrt{r})$. In $K_{4}, s O_{K_{4}}=\mathfrak{a}^{2} \mathfrak{b b}^{\prime}$ with $N(\mathfrak{a})=N(\mathfrak{b})=N\left(\mathfrak{b}^{\prime}\right)=s$. Exchanging $\mathfrak{b}$ and $\mathfrak{b}^{\prime}$ if necessary we may assume that $\mathfrak{b}$ is ramified in $K_{8} / K_{4}$. Since $h_{M_{16}}^{-}=\frac{Q_{M_{16}}}{2}\left(\frac{h_{K_{8}}^{-}}{Q_{K_{8}}}\right)^{2}$, if $h_{M_{16}}^{-}=2$, then $h_{K_{8}}^{-} / Q_{K_{8}}=2$ and $Q_{M_{16}}=1$. It follows that if $h_{M_{16}}^{-}=2$, then the unique ramified prime ideals in $K_{8} / K_{4}$ are $(p)$ and $\mathfrak{b}$, and $Q_{K_{8}}=1$. Note that $p$ is inert in $\mathbb{Q}(\sqrt{r})$ and in $\mathbb{Q}(\sqrt{s})$. Hence, if $h_{M_{16}}^{-}=2$, then $N_{M_{16}^{+} / \mathbb{Q}}\left(\mathfrak{p p}^{\prime}\right)^{1 / 8}=p \leq N_{M_{16}^{+} / \mathbb{Q}}(\mathfrak{D})^{1 / 8} \leq C$ and $\left(\frac{r}{p}\right)=\left(\frac{s}{p}\right)=-1$. According to Hecke's Theorem $\mathcal{F}\left(K_{8} / K_{4}\right)=(p) \mathfrak{b}$ for $p \neq 2, \mathcal{F}\left(K_{8} / K_{4}\right)=(2)^{e} \mathfrak{b}$ with $e=2$ or 3 (indeed, we will see below $e=3$ ) in the case that $p=2$ and $M_{16}^{+}$is the real subfield $M^{+}$of the fields numbered from 2 to 5 in Table 1. (See Theorem 10.2.9 in [Co] and Theorem 119 in [He.) We may assume that $s$ is odd. 
TABLE 2 .

\begin{tabular}{|c|c|c|c|c|c|}
\hline$M^{+}$ & $\mathrm{C}$ & $p$ & $K_{4}=K_{8}^{+}$ & $\mathcal{F}\left(K_{8} / K_{4}\right)$ & $h_{K_{8}}^{-}$ \\
\hline $\mathbb{Q}(\sqrt{257}, \sqrt{17+4 \sqrt{2}})$ & 25 & 5 & $\mathbb{Q}(\sqrt{17+4 \sqrt{2}})$ & $(5) \mathfrak{b}$ & 274 \\
\hline $\mathbb{Q}(\sqrt{101}, \sqrt{11+2 \sqrt{5}})$ & 25 & 2 & $\mathbb{Q}(\sqrt{11+2 \sqrt{5}})$ & $(2)^{3} \mathfrak{b}$ & 34 \\
\hline $\mathbb{Q}(\sqrt{181}, \sqrt{(27+\sqrt{5}) / 2})$ & 22 & 2 & $\mathbb{Q}(\sqrt{(27+\sqrt{5}) / 2})$ & $(2)^{3} \mathfrak{b}$ & 146 \\
\cline { 5 - 6 } & & 17 & & $(17) \mathfrak{b}$ & 658 \\
\hline $\mathbb{Q}(\sqrt{53}, \sqrt{(15+\sqrt{13}) / 2})$ & 23 & 2 & $\mathbb{Q}(\sqrt{(15+\sqrt{13}}) / 2)$ & $(2)^{3} \mathfrak{b}$ & 82 \\
\cline { 5 - 6 } & & 5 & & $(5) \mathfrak{b}$ & 34 \\
\hline $\mathbb{Q}(\sqrt{61}, \sqrt{(19+3 \sqrt{13}) / 2})$ & 23 & 2 & $\mathbb{Q}(\sqrt{(19+3 \sqrt{13}) / 2})$ & $(2)^{3} \mathfrak{b}$ & 50 \\
\hline
\end{tabular}

In the case that $M_{16}^{+}$is the real subfield of the first field $\mathrm{M}$ in Table 1 we take $L=\mathbb{Q}(\sqrt{2}), K_{4}=\mathbb{Q}(\sqrt{17+4 \sqrt{2}})$ and $s=257$. Furthermore, we can get rid of $p$ with $p \equiv 3 \bmod 4$. In fact, the narrow class number $h_{4}^{+}$of $K_{4}$ is odd. There exists a totally positive element $\alpha$ in $K_{4}$ such that $\mathfrak{b}^{h_{4}^{+}}=(\alpha)$ and $K_{4}(\sqrt{-\alpha})$ is contained in $H i l_{\text {nar }}\left(k_{2}\right)$. Since $s$ is odd and $\mathfrak{b}$ is the unique prime ideal ramified in $K_{4}(\sqrt{-\alpha}) / K_{4}$, the equation $x^{2} \equiv-\alpha \bmod \mathfrak{p}_{2}^{2}$ is solvable for each prime $\mathfrak{p}_{2}$ lying above 2 . We can write $K_{8}=K_{4}(\sqrt{-\alpha p})$. Hence, if $p=2$, then $\mathcal{F}\left(K_{8} / K_{4}\right)=(2)^{3} \mathfrak{b}$. If $p \neq 2$, then the prime ideal(s) lying above 2 is(are) not ramified in $K_{8} / K_{4}$ and $x^{2} \equiv-\alpha p \bmod \mathfrak{p}_{2}^{2}$ is solvable. It follows that $p \not \equiv 3 \bmod 4$. Suppose that $p \equiv 3 \bmod$ 4. Then $x^{2} \equiv-1 \bmod \mathfrak{p}_{2}^{2}$ would be solvable. This implies that the prime ideal(s) lying above 2 is(are) not ramified in $K_{4}(\sqrt{-1}) / K_{4}$. This contradicts the fact that $h_{4}^{+}$is odd.

(iii) For these $p \not \equiv 3 \bmod 4$ such that $p \leq C$ and $\left(\frac{r}{p}\right)=\left(\frac{s}{p}\right)=-1$ we have computed the relative class numbers of $K_{8}=K_{4}(\sqrt{-\alpha p})$ by using the technique developed in Lou7. In all there are nine such fields $K_{8}$ and we have verified that $h_{K_{8}}^{-}>2$, which permits us to conclude $h_{M_{16}}^{-}>2$ and $h_{N}^{-} \geq 2$. We give our computational results in Table 2.

Proposition 13. If $G\left(M_{16.1} / \mathbb{Q}\right)=D_{16}$ and $G\left(M_{16.2} / \mathbb{Q}\right)=G_{5}$, then $h_{N}^{-} \geq 2$.

Proof. Since $h_{M_{16.2}}^{-} \geq 2$, it follows from Proposition 10 and Theorem 3 that if there exists a field $N=M_{16.1} M_{16.2}$ with $h_{N}^{-}=1$, then $h_{M_{16.1}}^{-}=1$. Moreover, the unique quartic normal subfield $\mathbb{Q}\left(\sqrt{m_{1}}, \sqrt{m_{2}}\right)$ of $M_{16.1}^{+}=M_{16.2}^{+}$is contained in the octic quaternion subfield $M_{8.2}$ of $M_{16.2}$. Since the subfields $\mathbb{Q}\left(\sqrt{m_{1}}, \sqrt{m_{2}}\right)$ of the fields in Table 1 satisfy $\left(\frac{m_{1}}{m_{2}}\right)=\left(\frac{m_{2}}{m_{1}}\right)=+1$, there are at least four ramified prime ideals in $M_{8.2} / \mathbb{Q}\left(\sqrt{m_{1}}, \sqrt{m_{2}}\right)$, whence $2^{3} \mid h_{M_{8.2}}^{-}$and $h_{N}^{-}>1$. This is a contradiction.

Proposition 14. If $G\left(M_{16.1} / \mathbb{Q}\right)=D_{16}$ and $G\left(M_{16.2} / \mathbb{Q}\right)=G_{6}$, then $h_{N}^{-} \geq 2$.

Proof. Let $M_{16.2}$ be a compositum of two normal octic CM-fields $M_{8.1}$ and $M_{8.2}$ such that $M_{8.1}^{+}=M_{8.2}^{+}, G\left(M_{8.1} / \mathbb{Q}\right)=D_{8}$, and $G\left(M_{8.2} / \mathbb{Q}\right)=C_{4}^{*} \times C_{2}$. According to Proposition 10 if there exists a field $N=M_{16.1} M_{16.2}$ of $h_{N}^{-}=1$, then either $h_{M_{16.1}}^{-}=1$ or $h_{M_{16.2}}^{-}=1$. First, we verify that there is no pair of fields 
$\left(M_{16.1}, M_{16.2}\right)$ such that $h_{M_{16.1}}^{-}=1$ and $h_{M_{16.2}}^{-}=1,2$, or 4 . Indeed, we verify that there is no real bicyclic biquadratic field $\mathbb{Q}\left(\sqrt{m_{1}}, \sqrt{m_{2}}\right)$ which is a subfield of a dihedral CM-field $M_{16.1}$ with $h_{M_{16.1}}^{-}=1$ and at the same time is contained in the intersection of an octic dihedral CM-field $M_{8.1}$ with $h_{M_{8.1}}^{-}=1,2$, or 4 and an imaginary abelian octic field $M_{8.2}$ with $G\left(M_{8.2} / \mathbb{Q}\right)=C_{4}^{*} \times C_{2}$ and $h_{M_{8.2}}^{-}=1,2$, or $4([\mathrm{YK}]$ and $\mathrm{CK}]$ ). Second, we will show that there is no pair of fields $\left(M_{16.1}, M_{16.2}\right)$ such that $h_{M_{16.1}}^{-}=1,2$, or 4 and $h_{M_{16.2}}^{-}=1$. The unique field $M_{16.2}$ with $h_{M_{16.2}}^{-}=1$ is $\mathbb{Q}(\sqrt{17}, \sqrt{-(9+\sqrt{13}) / 2}, \sqrt{-(13+2 \sqrt{13})})$. Set $M_{8}^{+}=M_{16.2}^{+}=\mathbb{Q}(\sqrt{17}, \sqrt{(13+2 \sqrt{13})(9+\sqrt{13}) / 2})$. Suppose that there exists a field $M_{16.1}$ containing $M_{8}^{+}$with $G\left(M_{16.1} / \mathbb{Q}\right)=D_{16}$. We have $(13)=\mathcal{I}_{1}^{4} \mathcal{I}_{2}^{4}$ and $(17)=\mathcal{J}_{1}^{2} \mathcal{J}_{2}^{2}$ in $M_{8}^{+}$. Since $M_{8}^{+} / \mathbb{Q}(\sqrt{17})$ is cyclic, $\mathcal{I}_{1}$ and $\mathcal{I}_{2}$ are ramified in $M_{16.1} / M_{8}^{+}$. We claim that $\mathcal{J}_{1}$ and $\mathcal{J}_{2}$ are ramified in $M_{16.1} / M_{8}^{+}$. Suppose that $\mathcal{J}_{1}$ and $\mathcal{J}_{2}$ remain prime in $M_{16.1}$. Then $G_{-1}\left(\mathcal{J}_{1}\right)=G\left(M_{16.1} / \mathbb{Q}(\sqrt{13})\right)=D_{8}, G_{0}\left(\mathcal{J}_{1}\right)$ is of order 2 , and $G_{-1}\left(\mathcal{J}_{1}\right) / G_{0}\left(\mathcal{J}_{1}\right)$ is cyclic. In $D_{8}$ there is no normal subgroup of order 2 such that its factor group is cyclic of order 4 . Suppose now that $\mathcal{J}_{1}$ and $\mathcal{J}_{2}$ split in $M_{16.1} / M_{8}^{+}$. Write $(17)=\prod_{i=1}^{4} \mathfrak{P}_{i}{ }^{2}$ in $M_{16.1},(17)=\mathfrak{p p}^{\prime}$ in $\mathbb{Q}(\sqrt{13})$, and $(17)=\mathfrak{P P}^{\prime 2}$ in $L_{4}=\mathbb{Q}(\sqrt{(13+2 \sqrt{13})(9+\sqrt{13}) / 2})$. Assume that $\mathfrak{p} \mid \mathfrak{P}_{1}$. The decomposition field of $\mathfrak{P}_{1}$ in the extension $M_{16.1} / \mathbb{Q}(\sqrt{13})$ must be one of the three fields $L_{4}, \mathbb{Q}(\sqrt{13}, \sqrt{17})$ or $L_{4}^{\prime}=\mathbb{Q}(\sqrt{(13-2 \sqrt{13})(9-\sqrt{13}) / 2})$. Note that $\mathfrak{p}$ is inert in $L_{4} / \mathbb{Q}(\sqrt{13})$ and ramified in $\mathbb{Q}(\sqrt{13}, \sqrt{14}) / \mathbb{Q}(\sqrt{13})$ and $L_{4}^{\prime} / \mathbb{Q}(\sqrt{13})$. This is a contradiction. Consequently, if there is a CM-field $M_{16.1}$ containing $M_{8}^{+}$with $G\left(M_{16.1} / \mathbb{Q}\right)=D_{16}$, then $2^{3} \mid h_{M_{16.1}}^{-}$. This completes the proof.

Proposition 15. If $G\left(M_{16.1} / \mathbb{Q}\right)=D_{16}$ and $G\left(M_{16.2} / \mathbb{Q}\right)=G_{7}$, then $h_{N}^{-} \geq 2$.

Proof. Let $M_{16.2}=M_{8.1} M_{8.2}$ be a compositum of two normal octic CM-fields $M_{8.1}$ and $M_{8.2}$ such that $M_{8.1}^{+}=M_{8.2}^{+}, G\left(M_{8.1} / \mathbb{Q}\right)=D_{8}$, and $G\left(M_{8.2} / \mathbb{Q}\right)=C_{2}^{*} \times C_{2} \times C_{2}$. According to Proposition 10 if $h_{N}^{-}=1$, then $h_{M_{16.1}}^{-}=1$ or $h_{M_{16.2}}^{-}=1$.

Case (i) : Assume that $h_{M_{16.1}}^{-}=1$. According to [LO2] if $h_{M_{16.1}}^{-}=1$, then the biquadratic bicyclic subfield $K$ of $M_{16.1}^{+}$satisfies $K \in A:=\left\{\mathbb{Q}\left(\sqrt{m}_{1}, \sqrt{m}_{2}\right) ;\left(m_{1}, m_{2}\right)\right.$ $\in\{(2,257),(5,101),(5,181),(13,53),(13,61)\}\}$. Using $\mathrm{YK}$ we verify that $M_{8.1}=$ $\mathbb{Q}(\sqrt{5}, \sqrt{101}, \sqrt{-(33+6 \sqrt{5})})$, of which $h_{M_{8.1}}^{-}=2$, is the only dihedral octic CMfield $M_{8.1}$ such that $M_{8.1}^{+} \in A$ and $h_{M_{8.1}}^{-}$divides 4 . The compositum of $M_{8.1}$ and $\mathbb{Q}(\sqrt{11+2 \sqrt{5}}, \sqrt{101})$ contains the subfield $\mathbb{Q}(\sqrt{5}, \sqrt{101}, \sqrt{-3})$ whose relative class number is equal to 30 . Hence, we have shown that if $h_{M_{16.1}}^{-}=1$, then $h_{N}^{-} \geq 2$.

Case (ii): Assume that $h_{M_{16.2}}^{-}=1$. According to Lou3 there are three such fields $M_{16.2}$ : the fields numbered 6,7 , and 8 in Table 1 . For these three fields $M_{16.2}$ we examine whether there exists a normal CM-field $M_{16.1}$ such that $M_{16.1}^{+}=$ $M_{16.2}^{+}, G\left(M_{16.1} / \mathbb{Q}\right)=D_{16}$, and at most two prime ideals are ramified in the extension $M_{16.1} / M_{16.1}^{+}$.

(ii-a) Assume that $M_{16.2}$ is the field numbered 6 in Table 1 . In the proof of Proposition 11, part (i), and Lemma 7, point (1), we verified that there is no dihedral CM-field $M_{16.1}$ of degree 16 containing $M_{16.2}^{+}=L_{8}:=\mathbb{Q}(\sqrt{2}, \sqrt{3+\sqrt{3}})$ such that the only ramified prime ideals in $M_{16.1} / L_{8}$ lie above 2 or 3 . Hence if 
there is a dihedral CM-field $M_{16.1}$ of degree 16 containing $L_{8}$, then at least four prime ideals are ramified in the extension $M_{16.1} / L_{8}$, whence $8 \mid h_{M_{16.1}}$. Because if a prime $p$ with $p \neq 2,3$ divides $d_{M_{16.1}}$, then there are at least two prime ideals lying above $p$ which are ramified in $M_{16.1} / L_{8}$.

(ii-b) Assume that $M_{16.2}$ is the field numbered 7 in Table 1. If there is a dihedral CM-field $M_{16.1}$ of degree 16 containing $M_{16.2}^{+}=L_{8}^{\prime}:=\mathbb{Q}(\sqrt{11}, \sqrt{15+8 \sqrt{3}})$, then the prime ideal lying above 3 is ramified in the cyclic extension $M_{16.1} / \mathbb{Q}(\sqrt{11})$ and $Q_{M_{16.1}}=1$. In this case $h_{N}^{-}=1$ implies that $h_{M_{16.1}}^{-}=2, \mathcal{F}\left(M_{16.1} / L_{8}^{\prime}\right)=\mathcal{I}$, where $\mathcal{I}$ is the unique prime ideal lying above 3 in $L_{8}^{\prime}$. According to Lemma 7 , point (2), there is no dihedral CM-field $M_{16.1}$ of degree 16 containing $L_{8}^{\prime}$ with $\mathcal{F}\left(M_{16.1} / L_{8}^{\prime}\right)=\mathcal{I}$.

(ii-c) Assume that $M_{16.2}$ is the field numbered 8 in Table 1. Suppose that there is a dihedral CM-field $M_{16.1}$ of degree 16 containing

$$
M_{16.2}^{+}=L_{8}^{\prime \prime}=\mathbb{Q}(\sqrt{2}, \sqrt{(15+3 \sqrt{17}) / 2}) .
$$

Since two prime ideals $\mathcal{J}_{1}$ and $\mathcal{J}_{2}$ lying above 3 are ramified in the extension $L_{8}^{\prime \prime} / \mathbb{Q}(\sqrt{2}, \sqrt{17})$, they are totally ramified in the extension $M_{16.1} / \mathbb{Q}(\sqrt{2}, \sqrt{17})$, which implies $Q_{M_{16.1}}=1$. Hence, if $h_{N}^{-}=1$, then $h_{M_{16.1}}^{-}=2$ and $\mathcal{F}\left(M_{16.1} / L_{8}^{\prime \prime}\right)=\mathcal{J}_{1} \mathcal{J}_{2}$. According to Lemma 7 , point (3), there is no normal CM-field $M_{16.1}$ containing $L_{8}^{\prime \prime}$ with $\mathcal{F}\left(M_{16.1} / L_{8}^{\prime \prime}\right)=\mathcal{J}_{1} \mathcal{J}_{2}$.

The proof is complete.

In Propositions 16-18 below we deal with the composita $N=M_{16.1} M_{16.2}$ such that $G\left(M_{16 . i} / \mathbb{Q}\right) \in\left\{G_{5}, G_{6}, G_{7}\right\}$ for $i=1,2$.

Lemma 9. Let $L$ be an octic quaternion $C M$-field.

(1) ([Lou2, Corollary 10]) $h_{L}^{-} \equiv 2 \bmod 4$ if and only if $L$ is a pure quaternion CM-field with $L^{+}=\mathbb{Q}(\sqrt{2}, \sqrt{q})$, where $q \equiv 3 \bmod 8$ is prime.

(2) If $h_{L}^{-} \equiv 4 \bmod 8$, then $L$ is a pure quaternion extension of $\mathbb{Q}$ and $L^{+}=$ $\mathbb{Q}\left(\sqrt{2 p_{1}}, \sqrt{p_{2}}\right)$, where $p_{1} \equiv 3 \bmod 4$ and $p_{2} \equiv 3 \bmod 8$ are prime.

Proof. (2) Assume that $h_{L}^{-} \equiv 4 \bmod 8$. Counting the number of ramified prime ideals in $L$ it can be easily shown that $L$ is a pure quaternion extension of $\mathbb{Q}$. First we prove that the number of prime divisors of $d_{L}$ is equal to 3 and for each prime divisor $p$ of $d_{L}$ there is only one prime ideal lying above $p$ in $L$. Suppose that the number of prime divisors of $d_{L}$ is equal to 2 . Then $L^{+}$is of the form $\mathbb{Q}(\sqrt{2}, \sqrt{p}), \mathbb{Q}(\sqrt{2}, \sqrt{q})$, or $\mathbb{Q}(\sqrt{r}, \sqrt{s})$, where $p, q, r$, and $s$ are odd primes with $q \equiv 3 \bmod 8, p \equiv 1 \bmod$ $8, r, s \equiv 1 \bmod 4$, and $\left(\frac{s}{r}\right)=+1\left(\right.$ see $\left[\mathrm{K}, 2.2\right.$ Proposition]). If $L^{+}=\mathbb{Q}(\sqrt{2}, \sqrt{q})$, then $h_{L}^{-} \equiv 2 \bmod 4$. If either $L^{+}=\mathbb{Q}(\sqrt{2}, \sqrt{p})$ or $L^{+}=\mathbb{Q}(\sqrt{r}, \sqrt{s})$, then $h_{L}^{-} \equiv 0 \bmod 8$. Second, we claim that 2 must be ramified. Otherwise we have $L^{+}=\mathbb{Q}\left(\sqrt{p_{1}}, \sqrt{p_{2} p_{3}}\right)$, where $p_{1}, p_{2}$, and $p_{3}$ are odd primes with $p_{1} \equiv 1 \bmod 4$ and $p_{2} p_{3} \equiv 1 \bmod 4$. Since $\mathbb{Q}\left(\sqrt{p}_{1}, \sqrt{p_{2} p_{3}}\right)$ is embedded in an octic quaternion field, $\left(\frac{-1}{p_{1}}\right)=\left(\frac{p_{2} p_{3}}{p_{1}}\right)=+1$. It follows that $p_{1}$ splits in $\mathbb{Q}\left(\sqrt{p_{2} p_{3}}\right)$, two prime ideals lying above $p_{1}$ in $L^{+}$are ramified in $L / L^{+}$, whence $h_{L}^{-} \equiv 0 \bmod 8$. Write $L^{+}=\mathbb{Q}\left(\sqrt{m}_{1}, \sqrt{m_{2}}\right)$, where $m_{1}$ and $m_{2}$ are square free positive integers. Third, we verify that 2 is totally ramified. Suppose the contrary. Say 2 is not ramified in $\mathbb{Q}\left(\sqrt{m}_{2}\right)$. Then either $\left(m_{1}, m_{2}\right)=\left(2, p_{1} p_{2}\right)$ or $\left(m_{1}, m_{2}\right)=(2 p, q)$, where $p_{1}, p_{2}, p$, and $q$ are odd primes satisfying $p_{1} p_{2} \equiv 1$ $\bmod 4,\left(\frac{-1}{p_{1}}\right)=\left(\frac{2}{p_{1}}\right),\left(\frac{-1}{p_{2}}\right)=\left(\frac{2}{p_{2}}\right), q \equiv 1 \bmod 4,\left(\frac{-1}{q}\right)=\left(\frac{2 p}{q}\right)$, and $\left(\frac{-1}{p}\right)=\left(\frac{q}{p}\right)$. Note that $\mathbb{Q}(\sqrt{p}, \sqrt{q})$ with $p \equiv 3 \bmod 4$ and $q \equiv 1 \bmod 4$ cannot be embedded 
in an octic quaternion field. If $L^{+}=\mathbb{Q}\left(\sqrt{2}, \sqrt{p_{1} p_{2}}\right)$ and if $\left(\frac{-1}{p_{1}}\right)=\left(\frac{2}{p_{1}}\right)=1$ or $\left(\frac{-1}{p_{2}}\right)=\left(\frac{2}{p_{2}}\right)=1$, then $h_{L}^{-} \equiv 0 \bmod 8$. If $L^{+}=\mathbb{Q}\left(\sqrt{2}, \sqrt{p_{1} p_{2}}\right),\left(\frac{-1}{p_{1}}\right)=\left(\frac{2}{p_{1}}\right)=-1$, and $\left(\frac{-1}{p_{2}}\right)=\left(\frac{2}{p_{2}}\right)=-1$, then $p_{1} \equiv p_{2} \equiv 3 \bmod 8,2$ splits in $\mathbb{Q}\left(\sqrt{p_{1} p_{2}}\right)$, and $h_{L}^{-} \equiv 0$ $\bmod 8$. If $L^{+}=\mathbb{Q}(\sqrt{2 p}, \sqrt{q})$ with $q \equiv 1 \bmod 4$, then $\left(\frac{-1}{q}\right)=\left(\frac{2 p}{q}\right)=1$ and $h_{L}^{-} \equiv 0$ mod 8. Finally, the fact that 2 is totally ramified implies that $\left(m_{1}, m_{2}\right)=(2 p, q)$ with $q \equiv 3 \bmod 4,\left(\frac{-1}{p}\right)=\left(\frac{q}{p}\right)=-1$, and $\left(\frac{-1}{q}\right)=\left(\frac{2 p}{q}\right)=-1$. Thus $p \equiv 3 \bmod 4$ and $q \equiv 3 \bmod 8$.

Proposition 16. (1) There is one and only one CM-field $M_{16}$ of degree 16 with $G\left(M_{16} / \mathbb{Q}\right)=G_{5}$ such that $G\left(M_{16}^{+} / \mathbb{Q}\right)=D_{8}$ and every CM-subfield $L$ of $M_{16}$ has $h_{L}^{-} \mid 4: M_{16}=\mathbb{Q}(\sqrt{3+\sqrt{3}}, \sqrt{-(2+\sqrt{2})})$ with $h_{M_{16}}^{-}=2$ and $Q_{M_{16}}=2$.

(2) If $G\left(M_{16.1} / \mathbb{Q}\right)=G\left(M_{16.2} / \mathbb{Q}\right)=G_{5}$, and $M_{16.1}^{+}=M_{16.2}^{+}$with $G\left(M_{16.1}^{+} / \mathbb{Q}\right)$ $=D_{8}$, then $h_{N}^{-} \geq 2$.

(3) If $G\left(M_{16.1} / \mathbb{Q}\right)=G_{5}, G\left(M_{16.2} / \mathbb{Q}\right)=G_{6}$, and $M_{16.1}^{+}=M_{16.2}^{+}$with $G\left(M_{16.1}^{+} / \mathbb{Q}\right)=D_{8}$, then $h_{N}^{-} \geq 2$.

(4) If $G\left(M_{16.1} / \mathbb{Q}\right)=G_{5}, G\left(M_{16.2} / \mathbb{Q}\right)=G_{7}$, and $M_{16.1}^{+}=M_{16.2}^{+}$with $G\left(M_{16.1}^{+} / \mathbb{Q}\right)=D_{8}$, then there is exactly one field $N$ with $h_{N}^{-}=1$ :

$$
\mathbb{Q}(\sqrt{(2+\sqrt{2})}, \sqrt{3+\sqrt{3}}, \sqrt{-1}) .
$$

Moreover this field has class number one.

Proof. (1) The field $M_{16}=M_{8.1} M_{8.2}$ is a compositum of two octic CM-fields with $G\left(M_{8.1} / \mathbb{Q}\right)=C_{4}^{*} \times C_{2}$ and $h_{M_{8.1}}^{-} \mid 4, G\left(M_{8.2} / \mathbb{Q}\right)=Q_{8}$ and $h_{M_{8.2}}^{-} \mid 4$, and $M_{8.1}^{+}=M_{8.2}^{+}$. Now, out of the 51 such $M_{8.1}$ 's (see [CK] $), M_{8.1}=\mathbb{Q}(\sqrt{3}, \sqrt{-(2+\sqrt{2})})$ is the only one such that $M_{8.1}^{+}$can be embedded in an octic quaternion field $M_{8.2}$ with $h_{M_{8.2}}^{-} \mid 4$ (use Lemma 9), and we have $M_{8.2}=\mathbb{Q}(\sqrt{2}, \sqrt{3}, \sqrt{-(2+\sqrt{2})(3+\sqrt{2})}$ ). Since $h_{M_{8.1}}^{-}=Q_{M_{8.1}}=2, h_{M_{8.2}}^{-}=2, Q_{M_{8.2}}=1, w_{M_{8.2}}=2, w_{M_{16}}=w_{M_{8.1}}$, and $Q_{M_{16}}=2\left(\right.$ for $Q_{M_{8.1}}=2$ divides $Q_{M_{16}}$ ), we obtain $h_{M_{16}}^{-}=2$ by $(\mathrm{J})$.

(2) follows immediately from (1).

(3) and (4) Set $M_{16.1}=\mathbb{Q}(\sqrt{-(2+\sqrt{2})}, \sqrt{3+\sqrt{3}})$. The field $M_{16.2}=L_{8.1} L_{8.2}$ is a compositum of two octic CM-fields $L_{8.1}$ and $L_{8.2}$ such that $G\left(L_{8.1} / \mathbb{Q}\right)$ $=C_{4}^{*} \times C_{2}$ if $G\left(M_{16.2} / \mathbb{Q}\right)=G_{6}, G\left(L_{8.1} / \mathbb{Q}\right)=C_{2}^{*} \times C_{2} \times C_{2}$ if $G\left(M_{16.2} / \mathbb{Q}\right)$ $=G_{7}$, and $G\left(L_{8.2} / \mathbb{Q}\right)=D_{8}$. We look for all possible $N=M_{16.1} M_{16.2}$ with $h_{N}^{-}=1$. By [LO1 and $\mathrm{YK}$ there is only one octic dihedral CM-field $L_{8.2}$ such that $L_{8.2}^{+}=\mathbb{Q}(\sqrt{2}, \sqrt{3})$ and $h_{L_{8.2}}^{-} \mid 4: L_{8.2}=\mathbb{Q}(\sqrt{2}, \sqrt{-(3+\sqrt{3})})$ with $h_{L_{8.2}}^{-}=2$ and $Q_{L_{8.2}}=1$. For (3) we verify that there is only one imaginary abelian number field $L_{8.1}$ such that $G\left(L_{8.1} / \mathbb{Q}\right)=C_{4}^{*} \times C_{2}, L_{8.1}^{+}=\mathbb{Q}(\sqrt{2}, \sqrt{3})$, and $h_{L_{8.1}}^{-} \mid 4: L_{8.1}=\mathbb{Q}(\sqrt{3}, \sqrt{-(2+\sqrt{2})})([\mathrm{CK}])$. However, the compositum $\mathbb{Q}(\sqrt{-(2+\sqrt{2})}, \sqrt{-(3+\sqrt{3})})$ is not appropriate for us since its maximal real subfield is the octic quaternion field $\mathbb{Q}(\sqrt{2}, \sqrt{3}, \sqrt{(2+\sqrt{2})(3+\sqrt{3})})$. Consequently, there is no field $N$ with $h_{N}^{-}=1$. For (4) we find all imaginary abelian number fields $L_{8.1}$ such that $G\left(L_{8.1} / \mathbb{Q}\right)=C_{2}^{*} \times C_{2} \times C_{2}, L_{8.1}^{+}=\mathbb{Q}(\sqrt{2}, \sqrt{3})$, and $h_{L_{8.1}}^{-} \mid 4$. 
Such fields $L_{8.1}$ can be written of the form $L_{8.1}=\mathbb{Q}(\sqrt{2}, \sqrt{3}, \sqrt{-m})$, where $m=1$ or $m$ is a prime with $h_{\mathbb{Q}(\sqrt{-m})} \mid 4$. Otherwise, there are at least four ramified prime ideals in $L_{8.1} / L_{8.1}^{+}$and $h_{L_{8.1}}^{-} \equiv 0 \bmod 8$. We verify that there is precisely one such field : $L_{8.1}=\mathbb{Q}(\sqrt{2}, \sqrt{3}, \sqrt{-1})$ with $h_{L_{8.1}}^{-}=1$ and $Q_{L_{8.1}}=2$. Set $M_{16.2}=$ $\mathbb{Q}(\sqrt{2}, \sqrt{3+\sqrt{3}}, \sqrt{-1})$ and $N=M_{16.1} M_{16.2}=\mathbb{Q}(\sqrt{2+\sqrt{2}}, \sqrt{3+\sqrt{3}}, \sqrt{-1})$. Since $h_{M_{16.2}}^{-}=1, Q_{M_{16.2}}=2, w_{N}=w_{L}=48$ with $L=\mathbb{Q}(\sqrt{-(2+\sqrt{2})}, \sqrt{-1}, \sqrt{-3})$, $w_{M_{16.1}}=w_{F}=2$ with $F=\mathbb{Q}(\sqrt{3}, \sqrt{-(2+\sqrt{2})})$, and $w_{M_{16.2}}=w_{D}=24$ with $D=\mathbb{Q}(\sqrt{-1}, \sqrt{-2}, \sqrt{-3})$, we have $h_{N}^{-}=1$. (For $w_{L}, w_{F}$, and $w_{D}$ see Table II in [He.) By using [KT] we verify that the class number of $\mathbb{Q}(\sqrt{2+\sqrt{2}}, \sqrt{3+\sqrt{3}})$ is equal to one.

Remark 2. Assume that $M_{16.1}=M_{8.1} M_{8.2}$ and $M_{16.2}=L_{8.1} L_{8.2}$ are two composita of two normal octic CM-field with $M_{16.1}^{+}=M_{16.2}^{+}$and $M_{8.1}^{+}=M_{8.2}^{+}=L_{8.1}^{+}=$ $L_{8.2}^{+}$. Let $N=M_{16.1} M_{16.2}, R_{16.1}=M_{8.1} L_{8.1}, R_{16.2}=M_{8.2} L_{8.2}, T_{16.1}=M_{8.1} L_{8.2}$, and $T_{16.2}=L_{8.1} M_{8.2}$. We can easily verify that $M_{16.1}^{+} \neq R_{16.1}^{+}, M_{16.1}^{+} \neq T_{16.1}^{+}$, and $R_{16.1}^{+} \neq T_{16.1}^{+}$. Since $G\left(M_{16.1}^{+} / \mathbb{Q}\right), G\left(R_{16.1}^{+} / \mathbb{Q}\right)$, and $G\left(T_{16.1}^{+} / \mathbb{Q}\right)$ are the three factor groups of $G\left(N^{+} / \mathbb{Q}\right)$, we can determine $G\left(N^{+} / \mathbb{Q}\right)$ from these factor groups. Moreover, $R_{16.1}^{+}=R_{16.2}^{+}$and $T_{16.1}^{+}=T_{16.2}^{+}$.

(a) In Proposition $16(2)$ we have $G\left(R_{16.1} / \mathbb{Q}\right)=C_{4}^{*} \times C_{2} \times C_{2}, G\left(R_{16.2} / \mathbb{Q}\right)=$ $G_{8}, G\left(T_{16.1} / \mathbb{Q}\right)=G\left(T_{16.2} / \mathbb{Q}\right)=G_{5}, G\left(T_{16.1}^{+} / \mathbb{Q}\right)=D_{8}$, and $G\left(N^{+} / \mathbb{Q}\right)=$ $G_{7}$.

(b) In Proposition $16(3)$ we have $G\left(R_{16.1} / \mathbb{Q}\right)=C_{4}^{*} \times C_{4}^{*}, G\left(R_{16.2} / \mathbb{Q}\right)=G_{5}$, $G\left(T_{16.1} / \mathbb{Q}\right)=G_{6}, G\left(T_{16.2} / \mathbb{Q}\right)=G_{5}, G\left(T_{16.1}^{+} / \mathbb{Q}\right)=D_{8}$, and $G\left(N^{+} / \mathbb{Q}\right)=$ $G_{6}$ (cf. Proposition 27 (3) below).

(c) In Proposition $16(4)$ we have $G\left(R_{16.1} / \mathbb{Q}\right)=C_{4}^{*} \times C_{2}^{*} \times C_{2}, G\left(R_{16.2} / \mathbb{Q}\right)=$ $G_{5}, G\left(T_{16.1} / \mathbb{Q}\right)=G_{5}, G\left(T_{16.2} / \mathbb{Q}\right)=G_{8}, G\left(T_{16.1}^{+} / \mathbb{Q}\right)=Q_{8}$, and $G\left(N^{+} / \mathbb{Q}\right)$ $=G_{5}$ (cf. Propositions $19(2)$ and 19 (4) below).

The following remark will be needed in the sequel:

Remark 3. (a) There are eleven pairs of octic dihedral CM-fields $(L, M)$ such that $h_{L}^{-}\left|4, h_{M}^{-}\right| 4$, and $L^{+}=M^{+}=\mathbb{Q}\left(\sqrt{m_{1}}, \sqrt{m_{2}}\right):\left(m_{1}, m_{2}\right) \in S=$ $\{(2,7),(2,17),(5,11),(5,29),(3,13),(21,85),(10,65)\}$ ([LO1] and [YK]).

(b) Assume that $\left(m_{1}, m_{2}\right) \in S$. Let $l$ be a positive integer and

$$
F=\mathbb{Q}\left(\sqrt{m_{1}}, \sqrt{m_{2}}, \sqrt{-l}\right) .
$$

If $h_{F}^{-} \mid 4$ and all imaginary subfields of $\mathrm{F}$ have relative class numbers dividing 4 , then

$$
\begin{aligned}
\left(m_{1}, m_{2},-l\right) \in T=\{(2,7,-1),(2,7,-3), & (2,17,-1), \\
& (2,17,-3),(5,11,-1),(3,13,-1)\} .
\end{aligned}
$$

Proposition 17. (1) If $G\left(M_{16.1} / \mathbb{Q}\right)=G\left(M_{16.2} / \mathbb{Q}\right)=G_{6}$ and $G\left(M_{16.1}^{+} / \mathbb{Q}\right)=$ $D_{8}$, then $h_{N}^{-} \geq 2$.

(2) If $G\left(M_{16.1} / \mathbb{Q}\right)=G_{6}, G\left(M_{16.2} / \mathbb{Q}\right)=G_{7}$, and $G\left(M_{16.1}^{+} / \mathbb{Q}\right)=D_{8}$, then $h_{N}^{-} \geq 2$.

Proof. Let $M_{8.1}, M_{8.2}$ be two octic normal CM-fields such that $M_{16.1}=M_{8.1} M_{8.2}$, $M_{8.1}^{+}=M_{8.2}^{+}, G\left(M_{8.1} / \mathbb{Q}\right)=C_{4}^{*} \times C_{2}$, and $G\left(M_{8.2} / \mathbb{Q}\right)=D_{8}$. Let $L_{8.1}, L_{8.2}$ be two 
octic normal CM-fields such that $M_{16.2}=L_{8.1} L_{8.2}, L_{8.1}^{+}=L_{8.2}^{+}, G\left(L_{8.2} / \mathbb{Q}\right)=D_{8}$, $G\left(L_{8.1} / \mathbb{Q}\right)=C_{4}^{*} \times C_{2}$ or $C_{2}^{*} \times C_{2} \times C_{2}$, according to $G\left(M_{16.2} / \mathbb{Q}\right)=G_{6}$ or $G_{7}$. Then $M_{8.1}^{+}=M_{8.2}^{+}=L_{8.1}^{+}=L_{8.2}^{+}=\mathbb{Q}\left(\sqrt{m_{1}}, \sqrt{m_{2}}\right)$ is the unique quartic normal subfield of $M_{16.1}^{+}$. If $h_{N}^{-}=1$, then $\mathbb{Q}\left(\sqrt{m_{1}}, \sqrt{m_{2}}\right)$ is contained in two distinct octic dihedral CM-fields $M_{8.2}$ and $L_{8.2}$ with $h_{M_{8.2}}^{-} \mid 4$ and $h_{L_{8.2}}^{-} \mid 4$, hence $\left(m_{1}, m_{2}\right) \in S$.

(1) Assume that $G\left(M_{16.2} / \mathbb{Q}\right) \stackrel{=}{=} G_{6}$. Then $\mathbb{Q}\left(\sqrt{m_{1}}, \sqrt{m_{2}}\right)$ is contained in two abelian CM-fields $M_{8.1}$ and $L_{8.1}$ of Galois group $C_{4}^{*} \times C_{2}$, and the compositum $M_{8.1} L_{8.1}$ is an imaginary abelian number field with Galois group $C_{4}^{*} \times C_{4}^{*}$ or $C_{4}^{*} \times C_{2} \times C_{2}$. According to $\left[\mathrm{CK}\right.$ there is only one pair $\left(m_{1}, m_{2}\right)$ such that $h_{M_{8.1} L_{8.1}}^{-} \mid 4$ and $M_{8.1}^{+}=L_{8.1}^{+}=\mathbb{Q}\left(\sqrt{m_{1}}, \sqrt{m_{2}}\right)$ with $\left(m_{1}, m_{2}\right) \in S:\left(m_{1}, m_{2}\right)=$ $(5,29), M_{8.1}, L_{8.1} \in\{\mathbb{Q}(\sqrt{29}, \sqrt{-(5+2 \sqrt{5})}), \mathbb{Q}(\sqrt{5}, \sqrt{-(29+2 \sqrt{29})})\}$, and

$$
\begin{aligned}
& M_{8.2}, L_{8.2} \in\{\mathbb{Q}(\sqrt{29}, \sqrt{-(25+3 \sqrt{5}) / 2}) \\
&\mathbb{Q}(\sqrt{29}, \sqrt{-(7+2 \sqrt{5})}), \mathbb{Q}(\sqrt{29}, \sqrt{-(21+6 \sqrt{5})})\} .
\end{aligned}
$$

We verify that there is no quadruple of fields $\left(M_{8.1}, M_{8.2}, L_{8.1}, L_{8.2}\right)$ such that the composita $M_{8.1} M_{8.2}$ and $L_{8.1} L_{8.2}$ have the same maximal real subfield.

(2) Assume that $G\left(M_{16.2} / \mathbb{Q}\right)=G_{7}$. The compositum $M_{8.1} L_{8.1}$ is an imaginary abelian number field with Galois group $C_{4}^{*} \times C_{2}^{*} \times C_{2}$. By Remark 3 (b) we have $L_{8.1}=\mathbb{Q}\left(\sqrt{m_{1}}, \sqrt{m_{2}}, \sqrt{-l}\right)$ with $\left(m_{1}, m_{2},-l\right) \in T$. We verify that there are two quadruples of fields $\left(M_{8.1}, M_{8.2}, L_{8.1}, L_{8.2}\right)$ such that

(a) $h_{M_{8.1}}^{-}, h_{M_{8.2}}^{-}, h_{L_{8.1}}^{-}, h_{L_{8.2}}^{-} \in\{1,2,4\}$

(b) $M_{8.1}^{+}=M_{8.2}^{+}=L_{8.1}^{+}=L_{8.2}^{+}$, and

(c) $\left(M_{8.1} M_{8.2}\right)^{+}=\left(L_{8.1} L_{8.2}\right)^{+}, G\left(\left(M_{8.1} M_{8.2}\right)^{+} / \mathbb{Q}\right)=D_{8}$ :

$$
\begin{aligned}
& M_{8.1}=\mathbb{Q}(\sqrt{7}, \sqrt{-(2+\sqrt{2})}), M_{8.2}=\mathbb{Q}(\sqrt{7}, \sqrt{-(3+\sqrt{2}}), \\
& L_{8.1}=\mathbb{Q}(\sqrt{2}, \sqrt{7}, \sqrt{-1}), L_{8.2}=\mathbb{Q}(\sqrt{14}, \sqrt{-(4+\sqrt{2})}) ; \\
& M_{8.1}=\mathbb{Q}(\sqrt{7}, \sqrt{-(2+\sqrt{2})}), M_{8.2}=\mathbb{Q}(\sqrt{14}, \sqrt{-(4+\sqrt{2}}), \\
& L_{8.1}=\mathbb{Q}(\sqrt{2}, \sqrt{7}, \sqrt{-1}), L_{8.2}=\mathbb{Q}(\sqrt{7}, \sqrt{-(3+\sqrt{2})}) .
\end{aligned}
$$

For these two cases we verify that $h_{M_{16.1}}^{-}=4, h_{M_{16.2}}^{-}=2$, and $h_{N}^{-}=2 Q_{N} \geq 2$.

Remark 4. We keep the notation in the proof of Proposition 17. Let $R_{16.1}=$ $M_{8.1} L_{8.1}, R_{16.2}=M_{8.2} L_{8.2}, T_{16.1}=M_{8.1} L_{8.2}$, and $T_{16.2}=L_{8.1} M_{8.2}$.

(a) In Proposition 17 (1) there are three possibilities :

(i) If $G\left(N^{+} / \mathbb{Q}\right)=G_{7}$, then $G\left(R_{16.1} / \mathbb{Q}\right)=C_{4}^{*} \times C_{2} \times C_{2}, G\left(R_{16.2} / \mathbb{Q}\right)=$ $G_{7}, G\left(T_{16.1} / \mathbb{Q}\right)=G\left(T_{16.2} / \mathbb{Q}\right)=G_{6}$, and $G\left(T_{16.1}^{+} / \mathbb{Q}\right)=D_{8}(\mathrm{cf}$. Proposition 20 (3) below).

(ii) If $G\left(N^{+} / \mathbb{Q}\right)=G_{5}$, then $G\left(R_{16.1} / \mathbb{Q}\right)=C_{4}^{*} \times C_{4}^{*}, G\left(R_{16.2} / \mathbb{Q}\right)=G_{6}$, $G\left(T_{16.1} / \mathbb{Q}\right)=G\left(T_{16.2} / \mathbb{Q}\right)=G_{5}$, and $G\left(T_{16.1}^{+} / \mathbb{Q}\right)=Q_{8}$ (cf. Propositions $19(1)$ and $20(2)$ below).

(iii) If $G\left(N^{+} / \mathbb{Q}\right)=G_{6}$, then $G\left(R_{16.1} / \mathbb{Q}\right)=C_{4}^{*} \times C_{4}^{*}, G\left(R_{16.2} / \mathbb{Q}\right)=G_{6}$, $G\left(T_{16.1} / \mathbb{Q}\right)=G\left(T_{16.2} / \mathbb{Q}\right)=G_{6}$, and $G\left(T_{16.1}^{+} / \mathbb{Q}\right)=D_{8}($ cf. Proposition 28 (2) below). 
(b) In Proposition $17(2)$ we have $G\left(R_{16.1} / \mathbb{Q}\right)=C_{4}^{*} \times C_{2}^{*} \times C_{2}, G\left(R_{16.2} / \mathbb{Q}\right)=$ $G_{6}, G\left(T_{16.1} / \mathbb{Q}\right)=G_{6}, G\left(T_{16.2} / \mathbb{Q}\right)=G_{7}, G\left(T_{16.1}^{+} / \mathbb{Q}\right)=D_{8}$, and $G\left(N^{+} / \mathbb{Q}\right)$ $=G_{6}$ (cf. Proposition 28 (2) below).

We close this subsection with the following proposition.

Proposition 18. If $G\left(M_{16.1} / \mathbb{Q}\right)=G\left(M_{16.2} / \mathbb{Q}\right)=G_{7}$, then $h_{N}^{-} \geq 2$.

Proof. Let $M_{16.1}=M_{8.1} M_{8.2}$ and $M_{16.2}=L_{8.1} L_{8.2}$ such that $G\left(M_{8.1} / \mathbb{Q}\right)=$ $G\left(L_{8.1} / \mathbb{Q}\right)=C_{2}^{*} \times C_{2} \times C_{2}$, and $G\left(M_{8.2} / \mathbb{Q}\right)=G\left(L_{8.2} / \mathbb{Q}\right)=D_{8}$. Set $M_{8.1}^{+}=$ $M_{8.2}^{+}=L_{8.1}^{+}=L_{8.2}^{+}=\mathbb{Q}\left(\sqrt{m_{1}}, \sqrt{m_{2}}\right), M_{8.1}=\mathbb{Q}\left(\sqrt{m_{1}}, \sqrt{m_{2}}, \sqrt{-m_{0}}\right)$, and $L_{8.1}=$ $\mathbb{Q}\left(\sqrt{m_{1}}, \sqrt{m_{2}}, \sqrt{-l_{0}}\right)$. Suppose that $h_{N}^{-}=1$. Then $\left(m_{1}, m_{2}\right) \in S,\left(m_{1}, m_{2},-m_{0}\right) \in$ $T$, and $\left(m_{1}, m_{2},-l_{0}\right) \in T$, where $S$ and $T$ are as in Remark 3. Hence, $\left(m_{1}, m_{2}\right.$, $\left.-m_{0},-l_{0}\right) \in\{(2,7,-1,-3),(2,17,-1,-3)\}$. Since $h_{\mathbb{Q}(\sqrt{6}, \sqrt{-17})}^{-}=8$, we exclude $\left(m_{1}, m_{2},-m_{0},-l_{0}\right)=(2,17,-1,-3)$. There are two possible choices for quadruples $\left(M_{8.1}, M_{8.2}, L_{8.1}, L_{8.2}\right)$ :

$$
\begin{aligned}
& \text { (a) } \quad M_{8.1}=\mathbb{Q}(\sqrt{2}, \sqrt{7}, \sqrt{-1}), M_{8.2}=\mathbb{Q}(\sqrt{7}, \sqrt{-(3+\sqrt{2})}) \text {, } \\
& L_{8.1}=\mathbb{Q}(\sqrt{2}, \sqrt{7}, \sqrt{-3}) \text {, and } L_{8.2}=\mathbb{Q}(\sqrt{14}, \sqrt{-(4+\sqrt{2})}) ; \\
& \text { (b) } \quad M_{8.1}=\mathbb{Q}(\sqrt{2}, \sqrt{7}, \sqrt{-3}), M_{8.2}=\mathbb{Q}(\sqrt{7}, \sqrt{-(3+\sqrt{2})}) \text {, } \\
& L_{8.1}=\mathbb{Q}(\sqrt{2}, \sqrt{7}, \sqrt{-1}) \text {, and } L_{8.2}=\mathbb{Q}(\sqrt{14}, \sqrt{-(4+\sqrt{2})}) \text {. }
\end{aligned}
$$

However, in case (a) we have $M_{16.1}^{+}=\mathbb{Q}(\sqrt{3+\sqrt{2}}, \sqrt{7}) \neq \mathbb{Q}(\sqrt{3(4+\sqrt{2})}, \sqrt{14})=$ $M_{16.2}^{+}$, and in case (b) $M_{16.1}^{+}=\mathbb{Q}(\sqrt{3(3+\sqrt{2})}, \sqrt{7}) \neq \mathbb{Q}(\sqrt{(4+\sqrt{2})}, \sqrt{14})=M_{16.2}^{+}$. Consequently, $h_{N}^{-} \geq 2$.

Remark 5. We keep the notation in the proof of Propostion 18. Let $R_{16.1}=$ $M_{8.1} L_{8.1}, R_{16.2}=M_{8.2} L_{8.2}, T_{16.1}=M_{8.1} L_{8.2}$, and $T_{16.2}=L_{8.1} M_{8.2}$. In Proposition 18 we have $G\left(R_{16.1} / \mathbb{Q}\right)=C_{2}^{*} \times C_{2} \times C_{2} \times C_{2}, G\left(R_{16.2} / \mathbb{Q}\right)=G_{7}, G\left(T_{16.1} / \mathbb{Q}\right)=$ $G\left(T_{16.2} / \mathbb{Q}\right)=G_{7}, G\left(T_{16.1}^{+} / \mathbb{Q}\right)=D_{8}$, and $G\left(N^{+} / \mathbb{Q}\right)=G_{7}$ (cf. Proposition $20(4)$ below).

4.2. In this subsection we assume that $G\left(M_{8}^{+} / \mathbb{Q}\right)=Q_{8}$. Then $G\left(M_{16.1} / \mathbb{Q}\right)$, $G\left(M_{16.2} / \mathbb{Q}\right) \in\left\{G_{5}, G_{8}\right\}$.

Proposition 19. (1) If $G\left(M_{16.1} / \mathbb{Q}\right)=G\left(M_{16.2} / \mathbb{Q}\right)=G_{5}$, then $h_{N}^{-} \geq 2$.

(2) If $G\left(M_{16.1} / \mathbb{Q}\right)=G_{5}$ and $G\left(M_{16.2} / \mathbb{Q}\right)=G_{8}$, then there is only one compositum $N=M_{16.1} M_{16.2}$ with $h_{N}^{-}=1: N=\mathbb{Q}(\sqrt{2+\sqrt{2}}, \sqrt{3+\sqrt{3}}, \sqrt{-1})$.

(3) If $G\left(M_{16.1} / \mathbb{Q}\right)=G\left(M_{16.2} / \mathbb{Q}\right)=G_{8}$, then $h_{N}^{-} \geq 2$.

Proof. (1) This case is treated in Proposition 17 (1). (See Remark 4 (a) (ii).)

(2) This case is dealt with in Proposition 16 (4). (See Remark 2 (c).)

(3) To begin with we prove that if $K_{16}=A_{1} A_{2}$ is a compositum of two octic quaternion CM-fields $A_{1}$ and $A_{2}$ with the same maximal real subfield $A_{1}^{+}=A_{2}^{+}$, then the Galois group $G\left(K_{16} / \mathbb{Q}\right)$ is isomorphic to $G_{8}, G\left(K_{16}^{+} / \mathbb{Q}\right)=C_{2} \times C_{2} \times C_{2}$, and $h_{K_{16}}^{-} \geq 8$. From Proposition 9 it follows that $G\left(K_{16} / \mathbb{Q}\right)=G_{8}$ and $G\left(K_{16}^{+} / \mathbb{Q}\right)=C_{2} \times$ $C_{2} \times C_{2}$. Note that $h_{K_{16}}^{-}=\left(Q_{K_{16}} / 2\right) h_{A_{1}}^{-} h_{A_{2}}^{-}$and that there is only one octic quaternion CM-field with relative class number 2: $\mathbb{Q}(\sqrt{2}, \sqrt{3}, \sqrt{-(2+\sqrt{2})(3+\sqrt{3})})$ 
TABLE 3 .

$\begin{array}{cccc}N r . & M_{16} & Q_{M_{16}} & h_{M_{16}}^{-} \\ 1 & \mathbb{Q}(\sqrt{2}, \sqrt{17}, \sqrt{5}, \sqrt{-(5+2 \sqrt{2})}) & 2 & 1 \\ 2 & \mathbb{Q}(\sqrt{5}, \sqrt{29}, \sqrt{3}, \sqrt{-(7+2 \sqrt{5})}) & 1 & 2 \\ 3 & \mathbb{Q}(\sqrt{3}, \sqrt{13}, \sqrt{2}, \sqrt{-(5+\sqrt{13}) / 2}) & 2 & 4 \\ 4 & \mathbb{Q}(\sqrt{5}, \sqrt{11}, \sqrt{2}, \sqrt{-(7+\sqrt{5})}) & 2 & 4 \\ 5 & \mathbb{Q}(\sqrt{21}, \sqrt{85}, \sqrt{5}, \sqrt{-(37+7 \sqrt{21}) / 2}) & 2 & 4\end{array}$

([Lou2 $]$ ). Moreover, this is the only imaginary pure quaternion extension of $\mathbb{Q}$ containing $\mathbb{Q}(\sqrt{2}, \sqrt{3})$. If $A_{1}=\mathbb{Q}(\sqrt{2}, \sqrt{3}, \sqrt{-(2+\sqrt{2})(3+\sqrt{3})})$, then there are at least four ramified prime ideals in the extension $A_{2} / \mathbb{Q}(\sqrt{2}, \sqrt{3})$, whence $8 \mid h_{A_{2}}^{-}$and $8 \mid h_{K_{16}}^{-}$. If neither $A_{1}$ nor $A_{2}$ is equal to $\mathbb{Q}(\sqrt{2}, \sqrt{3}, \sqrt{-(2+\sqrt{2})(3+\sqrt{3})})$, then $h_{A_{1}}^{-}, h_{A_{2}}^{-} \geq 4$ and $h_{K_{16}}^{-} \geq 8 Q_{K_{16}}$. Let $M_{16.1}=M_{8.1} M_{8.2}$ and $M_{16.2}=L_{8.1} L_{8.2}$ with $G\left(M_{8.1} / \mathbb{Q}\right)=G\left(L_{8.1} / \mathbb{Q}\right)=C_{2}^{*} \times C_{2} \times C_{2}$ and $G\left(M_{8.2} / \mathbb{Q}\right)=G\left(L_{8.2} / \mathbb{Q}\right)=Q_{8}$. Then the relative class number of the compositum $M_{8.2} L_{8.2}$ is $\geq 8$, and $h_{N}^{-}>1$.

4.3. In this subsection we assume that $G\left(M_{8}^{+} / \mathbb{Q}\right)=C_{2} \times C_{2} \times C_{2}$. By Proposition 9 and the proof of Proposition 19, point (3), we exclude the normal CM-fields $M$ with $G(M / \mathbb{Q})=G_{8}$, hence $G\left(M_{16.1} / \mathbb{Q}\right), G\left(M_{16.2} / \mathbb{Q}\right) \in\left\{G_{7}, G_{9}, C_{4}^{*} \times C_{2} \times C_{2}, C_{2}^{*} \times\right.$ $\left.C_{2} \times C_{2} \times C_{2}\right\}$. We will prove that if $G\left(M_{8}^{+} / \mathbb{Q}\right)=C_{2} \times C_{2} \times C_{2}$, then $h_{N}^{-} \geq 2$.

For the proof of Proposition 20 we need the following remark:

Remark 6. According to [LO1] and [YK] there are five normal CM-fields $M_{16}$ with Galois group $G\left(M_{16} / \mathbb{Q}\right)=G_{7}$ such that $M_{16}=F_{8.1} F_{8.2}$ is a compositum of two octic dihedral CM-fields with $F_{8.1}^{+}=F_{8.2}^{+}$and $h_{F_{8.1}}^{-}, h_{F_{8.2}}^{-} \in\{1,2,4\}$. These fields are given in Table 3 .

Proposition 20. Assume that $G\left(M_{16 . i}^{+} / \mathbb{Q}\right)=C_{2} \times C_{2} \times C_{2}$ for $i=1,2$.

(1) If $G\left(M_{16.1} / \mathbb{Q}\right)=G\left(M_{16.2} / \mathbb{Q}\right)=G_{7}$, then $h_{N}^{-} \geq 2$.

(2) If $G\left(M_{16.1} / \mathbb{Q}\right)=G_{7}$ and $G\left(M_{16.2} / \mathbb{Q}\right)=G_{9}$, then $h_{N}^{-} \geq 2$.

(3) If $G\left(M_{16.1} / \mathbb{Q}\right)=G_{7}$ and $G\left(M_{16.2} / \mathbb{Q}\right)=C_{4}^{*} \times C_{2} \times C_{2}$, then $h_{N}^{-} \geq 2$.

(4) If $G\left(M_{16.1} / \mathbb{Q}\right)=G_{7}$ and $G\left(M_{16.2} / \mathbb{Q}\right)=C_{2}^{*} \times C_{2} \times C_{2} \times C_{2}$, then $h_{N}^{-} \geq 2$.

Proof. Let $M_{16.1}=M_{8.1} M_{8.2}$ with $G\left(M_{8.1} / \mathbb{Q}\right)=G\left(M_{8.2} / \mathbb{Q}\right)=D_{8}$ and $M_{8.1}^{+}=$ $M_{8.2}^{+}$.

(1) By Remark 6 there is no pair of $\left(M_{16.1}, M_{16.2}\right)$ such that $M_{16.1}=M_{8.1} M_{8.2}$ with $M_{8.1}^{+}=M_{8.2}^{+}$and $G\left(M_{8.1} / \mathbb{Q}\right)=G\left(M_{8.2} / \mathbb{Q}\right)=D_{8}, M_{16.2}=L_{8.1} L_{8.2}$ with $L_{8.1}^{+}=L_{8.2}^{+}$and $G\left(L_{8.1} / \mathbb{Q}\right)=G\left(L_{8.2} / \mathbb{Q}\right)=D_{8}, h_{M_{8.1}}^{-}, h_{M_{8.2}}^{-}, h_{L_{8.1}}^{-}, h_{L_{8.2}}^{-} \in\{1,2,4\}$, and $M_{16.1}^{+}=M_{16.2}^{+}$. It follows that if $G\left(M_{16.1} / \mathbb{Q}\right)=G\left(M_{16.2} / \mathbb{Q}\right)=G_{7}$, then $h_{N}^{-}>1$.

(2) Suppose that $h_{N}^{-}=1$. Then $M_{16.1}$ is one of the five fields $M_{16}$ in Table 3. According to [LO2] if $h_{M_{16.2}}^{-}=1$, then

$$
M_{16.2}=\mathbb{Q}(\sqrt{2}, \sqrt{5}, \sqrt{37}, \sqrt{-(2 \sqrt{2}+3 \sqrt{5})(2+\sqrt{5})}) .
$$


There is no field $M_{16}$ in Table 3 such that $M_{16}^{+}=\mathbb{Q}(\sqrt{2}, \sqrt{5}, \sqrt{37})$. So, by Proposition 10 if $h_{N}^{-}=1$, then $h_{M_{16.1}}^{-}=1$ and $h_{M_{16.2}}^{-}=2$ or 4 . We will examine whether there exists a normal CM-field $M_{16.2}$ containing $\mathbb{Q}(\sqrt{2}, \sqrt{5}, \sqrt{17})$ such that $G\left(M_{16.2} / \mathbb{Q}\right)=G_{9}$ and $h_{M_{16.2}}^{-}=2$ or 4 . Set $M_{16.2}^{+}=\mathbb{Q}(\sqrt{2}, \sqrt{5}, \sqrt{17})$. Let us determine which prime ideals can be ramified in $M_{16.2} / M_{16.2}^{+}$. If there exists a prime $p$ different from 2,5 , and 17 such that the prime ideals lying above $p$ are ramified in the extension $M_{16.2} / M_{16.2}^{+}$, then $8 \mid h_{M_{16.2}}^{-}$. In $M_{16.2}^{+}$we let $(2)=\mathcal{I}_{2}^{2} \mathcal{I}_{2}^{\prime 2},(5)=\mathcal{I}_{5}^{2} \mathcal{I}_{5}^{\prime 2}$, and $(17)=\mathcal{I}_{17}^{2} \mathcal{I}_{17}^{\prime}{ }^{2}$. If $h_{M_{16.2}}^{-}=2$ or 4 , then the number $t$ of ramified prime ideals in $M_{16.2} / M_{16.2}^{+}$is $\leq 3$. The first field in Table 3 is $\operatorname{Hil}_{\text {nar }}(\mathbb{Q}(\sqrt{2}, \sqrt{5}, \sqrt{17}))$, hence $t \neq 0$. Since $M_{16.2} / \mathbb{Q}$ is normal, $t=2$ and $\mathcal{F}\left(M_{16.2} / M_{16.2}^{+}\right)=\mathcal{I}_{2}^{e} \mathcal{I}_{2}^{\prime e}, \mathcal{I}_{5} \mathcal{I}_{5}^{\prime}$, or $\mathcal{I}_{17} \mathcal{I}_{17}^{\prime}$, where $e=2,4$, or 5 . According to Lemma 7, point (4), there is no such $M_{16.2}$. It follows that if $G\left(M_{16.1} / \mathbb{Q}\right)=G_{7}$ and $G\left(M_{16.2} / \mathbb{Q}\right)=G_{9}$, then $h_{N}^{-}>1$.

(3) This case is treated in Proposition 17 (1). (See Remark 4 (a) (i).)

(4) This case is treated in Proposition 19. (See Remark 5.)

Proposition 21. (1) If $G\left(M_{16.1} / \mathbb{Q}\right)=G\left(M_{16.2} / \mathbb{Q}\right)=G_{9}$, then $h_{N}^{-} \geq 2$.

(2) If $G\left(M_{16.1} / \mathbb{Q}\right)=G_{9}$ and $G\left(M_{16.2} / \mathbb{Q}\right)=C_{4}^{*} \times C_{2} \times C_{2}$, then $h_{N}^{-} \geq 2$.

(3) If $G\left(M_{16.1} / \mathbb{Q}\right)=G_{9}$ and $G\left(M_{16.2} / \mathbb{Q}\right)=C_{2}^{*} \times C_{2} \times C_{2} \times C_{2}$, then $h_{N}^{-} \geq 2$.

Proof. (1) From Proposition 10 it follows that if there exists a field $N$ with $h_{N}^{-}=1$, then $M_{16.1}$ or $M_{16.2}$, say $M_{16.1}$, satisfies $h_{M_{16.1}}^{-}=1$. Hence,

$$
M_{16.1}=\mathbb{Q}(\sqrt{2}, \sqrt{5}, \sqrt{37}, \sqrt{(2 \sqrt{2}+3 \sqrt{5})(2-\sqrt{5})})
$$

([LO2, Theorem 20]) and $M_{16.1}^{+}=\mathbb{Q}(\sqrt{2}, \sqrt{5}, \sqrt{37})$. We claim that $h_{M_{16.2}}^{-}>$ $2 Q_{M_{16.2}}$, whence $h_{N}^{-} \geq 2$. In $M_{16.1}^{+}(2)=\mathcal{I}_{2}^{2} \mathcal{I}_{2}^{\prime 2},(5)=\mathcal{I}_{5}^{2} \mathcal{I}_{5}^{\prime 2}$, and $(37)=\mathcal{I}_{37}^{2} \mathcal{I}_{37}^{\prime 2}$. Suppose that there exists a field $M_{16.2}$ with $M_{16.1}^{+}=M_{16.2}^{+}, h_{M_{16.2}}^{-}=2 Q_{M_{16.2}}$. By Theorem 2, point (3), at most two prime ideals are ramified in $M_{16.2} / M_{16.2}^{+}$. Note that $M_{16.1}=H_{i l} l_{n a r}\left(M_{16.1}^{+}\right)$. Hence, there are exactly two ramified prime ideals in $M_{16.2} / M_{16.2}^{+}$and $\mathcal{F}\left(M_{16.2} / M_{16.1}^{+}\right)=\mathcal{I}_{2}^{e} \mathcal{I}_{2}^{\prime e}$ with $e=2$, 4 , or $5, \mathcal{I}_{5} \mathcal{I}_{5}^{\prime}$, or $\mathcal{I}_{37} \mathcal{I}_{37}^{\prime}$. According to Lemma 7, point (5), there is no such $M_{16.2}$. It follows the conclusion.

(2) Suppose that $h_{N}^{-}=1$. Then, $M_{16.2}=\mathbb{Q}(\sqrt{-(5+2 \sqrt{5})}, \sqrt{2}, \sqrt{3})$, the only imaginary abelian number field with Galois group $C_{4}^{*} \times C_{2} \times C_{2}$ of relative class number dividing 4 (see [CK]). Since $h_{M_{16.2}}^{-}=4, h_{M_{16.1}}^{-}=1$ by Proposition 10 and $M_{16.1}$ is as in (1). Since $\mathbb{Q}(\sqrt{2}, \sqrt{5}, \sqrt{37})=M_{16.1}^{+} \neq M_{16.2}^{+}=\mathbb{Q}(\sqrt{2}, \sqrt{3}, \sqrt{5})$, we have a contradiction.

(3) Suppose that $h_{N}^{-}=1$. Since $h_{M_{16.2}}^{-}>1$ (see [CK]), by Proposition 10 we have $h_{M_{16.1}}^{-}=1$. Hence, $M_{16.1}$ is as in (1) and (2). Since at most two prime ideals of $M_{16.2}^{+}=M_{16.1}^{+}=\mathbb{Q}(\sqrt{2}, \sqrt{5}, \sqrt{37})$ are ramified in $M_{16.2} / M_{16.2}^{+}$, we have $M_{16.2}=\mathbb{Q}(\sqrt{2}, \sqrt{5}, \sqrt{37}, \sqrt{-1})$ and $240=h_{M_{16.2}}^{-} \mid\left(4 h_{N}^{-}\right)$. A contradiction.

4.4. In this subsection we assume that $G\left(M_{8}^{+} / \mathbb{Q}\right)=C_{4} \times C_{2}$. Then $G\left(M_{16.1} / \mathbb{Q}\right)$, $G\left(M_{16.2} / \mathbb{Q}\right) \in\left\{G_{4}, G_{5}, G_{6}, C_{4}^{*} \times C_{4}^{*}, C_{4}^{*} \times C_{2}^{*} \times C_{2}^{*}, C_{8}^{*} \times C_{2}\right\}$.

Proposition 22. If $G\left(M_{16.1} / \mathbb{Q}\right)=G_{4}$ and $G\left(M_{16.2} / \mathbb{Q}\right) \in\left\{G_{4}, G_{5}, C_{8}^{*} \times C_{2}\right\}$, then $h_{N}^{-} \geq 2$.

Proof. It follows from Theorem 3 and Propositions 10 and 24 below. 
Proposition 23. If $G\left(M_{16.1} / \mathbb{Q}\right)=G_{4}$ and $G\left(M_{16.2} / \mathbb{Q}\right)=G_{6}$, then there is one and only one field $N=M_{16.1} M_{16.2}$ with $h_{N}^{-}=1$. Namely, $N$ is the compositum of $M_{16.1}=\mathbb{Q}(\sqrt{2}, \sqrt{17+4 \sqrt{17}})(\theta)$ with $\theta^{8}+17 \theta^{6}+85 \theta^{4}+136 \theta^{2}+68=0$ and $M_{16.2}=\mathbb{Q}(\sqrt{2}, \sqrt{17}, \sqrt{-(5+\sqrt{17}) / 2}, \sqrt{-(17+3 \sqrt{17}) / 2})$. Moreover, $h_{N}=1$, $N^{+}=\mathbb{Q}(\sqrt{2}, \sqrt{17+4 \sqrt{17}})(\alpha)$ with $\alpha^{8}-2 \alpha^{7}-147 \alpha^{6}+218 \alpha^{5}+4966 \alpha^{4}-9058 \alpha^{3}-$ $14325 \alpha^{2}+25774 \alpha+1361=0, G\left(N^{+} / \mathbb{Q}\right)=C_{8} \times C_{2}, d_{N^{+}}=2^{24} 17^{14}$, and $d_{N}=$ $d_{N^{+}}^{2}=d_{M_{16.1}}^{2}=2^{48} 17^{28}$.

Proof. From Proposition 10 if $h_{N}^{-}=1$, then $h_{M_{16.1}}^{-}=2 Q_{M_{16.1}}, h_{M_{16.2}}^{-}=1, M_{16.2}=$ $\mathbb{Q}(\sqrt{2}, \sqrt{17}, \sqrt{-(5+\sqrt{17}) / 2}, \sqrt{-(17+3 \sqrt{17}) / 2})$ and $M_{16.2}^{+}=\mathbb{Q}(\sqrt{2}, \sqrt{17+4 \sqrt{17}})$ (see [Lou3]). Since $M_{16.1} / \mathbb{Q}(\sqrt{2})$ is cyclic, the two prime ideals lying above 17 are ramified in $M_{16.1} / M_{16.1}^{+}$with $M_{16.1}^{+}=\mathbb{Q}(\sqrt{2}, \sqrt{17+4 \sqrt{17}})$. If $h_{M_{16.1}}^{-}=2 Q_{M_{16.1}}$, then at most two prime ideals are ramified in $M_{16.1} / M_{16.1}^{+}$. Hence,

$$
\mathcal{F}\left(M_{16.1} / \mathbb{Q}(\sqrt{17+4 \sqrt{17}}, \sqrt{2})\right)=\mathcal{I}_{17} \mathcal{I}_{17}^{\prime},
$$

where $(17)=\mathcal{I}_{17}^{4} \mathcal{I}^{\prime 4}{ }_{17}$ in $\mathbb{Q}(\sqrt{2}, \sqrt{17+4 \sqrt{17}})$. Let $L_{8}$ be any one of two non-normal octic CM-subfields of $M_{16.1}$. Then $L_{8}^{+}=\mathbb{Q}(\sqrt{17+4 \sqrt{17}})$ or $\mathbb{Q}(\sqrt{17+\sqrt{17}})$. We claim that $L_{8}^{+}=\mathbb{Q}(\sqrt{17+4 \sqrt{17}})$. Suppose that $L_{8}^{+}=\mathbb{Q}(\sqrt{17+\sqrt{17}})$, the cyclic quartic field of conductor 136 . Then $M_{16.1} / \mathbb{Q}(\sqrt{17+4 \sqrt{17}})$ is cyclic, the two prime ideals lying above 2 would be ramified in $M_{16.1} / M_{16.1}^{+}$and $8 \mid h_{M_{16.1}}^{-}$. Since $h_{M_{16.1}}^{-}=$ $\frac{Q_{M_{16.1}}}{2}\left(\frac{h_{L_{8}}^{-}}{Q_{L_{8}}}\right)^{2}$, the fact that $h_{M_{16.1}}^{-}=2 Q_{M_{16.1}}$ implies that $h_{L_{8}}^{-}=2 Q_{L_{8}}$. Hence, the only ramified prime ideals in $L_{8} / L_{8}^{+}$are $\mathfrak{p}_{2}$ and $\mathfrak{p}_{17}$, where $(2)=\mathfrak{p}_{2} \mathfrak{p}_{2}^{\prime}$ and $(17)=\mathfrak{p}_{17}^{4}$ in $\mathbb{Q}(\sqrt{17+4 \sqrt{17}})$. We have $\mathcal{F}\left(L_{8} / L_{8}^{+}\right)=\mathfrak{p}_{2}^{e} \mathfrak{p}_{17}$ with $e=2$ or 3 . According to Lemma 7, point (6), we have $e=3$ and $L_{8}=\mathbb{Q}(\theta)$ with $\theta^{8}+17 \theta^{6}+85 \theta^{4}+136 \theta^{2}+$ $68=0$. We verify that $h_{L_{8}}^{-}=2$ by using the technique developed in [Lou7]. Hence $h_{M_{16.1}}^{-}=2$ and $h_{N}^{-}=1$. The conductor of the extension $N^{+} / \mathbb{Q}(\sqrt{2}, \sqrt{17+4 \sqrt{17}})$ is $\mathcal{I}_{17} \mathcal{I}_{17}^{\prime}$. We verify that $h_{N^{+}}=1$ and $G\left(N^{+} / \mathbb{Q}\right)=C_{8} \times C_{2}$.

The following result will be used in the sequel.

Proposition 24 ([CK]). (1) There are two imaginary abelian number fields with Galois group $C_{4}^{*} \times C_{4}^{*}$ and relative class numbers equal to one.

(2) There are seven imaginary abelian number fields with Galois group $C_{4}^{*} \times$ $C_{2}^{*} \times C_{2}^{*}$ and relative class numbers equal to one.

(3) There is one and only one field with Galois group $C_{8}^{*} \times C_{2}$ and relative class number dividing 4.

These fields are compiled in Table 4.

Proposition 25. If $G\left(M_{16.1} / \mathbb{Q}\right)=G_{4}$ and $G\left(M_{16.2} / \mathbb{Q}\right)=C_{4}^{*} \times C_{4}^{*}$, then $h_{N}^{-} \geq 2$.

Proof. If $h_{N}^{-}=1$, then $h_{M_{16.1}}^{-}=2 Q_{M_{16.1}}$ and $h_{M_{16.2}}^{-}=1$. There are two fields $M_{16.2}$ with $h_{M_{16.2}}^{-}=1$.

(i) Let us take $M_{16.2}=\mathbb{Q}(\sqrt{-(5+2 \sqrt{5})}, \sqrt{-(13+2 \sqrt{13})})$; then $M_{16.2}^{+}=F_{8}:=$ $\mathbb{Q}(\sqrt{5}, \sqrt{(65+\sqrt{65}) / 2})$ (see [CK] and [G]). We claim that $h_{M_{16.1}}>2 Q_{M_{16.1}}$ for 
TABLE 4. Here $\theta^{8}-\theta^{7}+10 \theta^{6}-11 \theta^{5}+15 \theta^{4}-61 \theta^{3}+58 \theta^{2}-47 \theta+103=$ 0 and $\mathbb{Q}(\theta)$ is the imaginary octic cyclic number field of conductor 51 (see $[\mathrm{PK}]$ ).

$\begin{array}{cccccc}N r . & M & G(M / \mathbb{Q}) & h_{M}^{-} & Q_{M} & h_{M^{+}} \\ 1 & \mathbb{Q}(\sqrt{-(5+2 \sqrt{5})}, \sqrt{-(13+2 \sqrt{13})}) & C_{4}^{*} \times C_{4}^{*} & 1 & 2 & 1 \\ 2 & \mathbb{Q}(\sqrt{-(5+2 \sqrt{5})}, \sqrt{-(2+\sqrt{2})}) & C_{4}^{*} \times C_{4}^{*} & 1 & 2 & 1 \\ 3 & \mathbb{Q}(\sqrt{-(5+2 \sqrt{5})}, \sqrt{-1}, \sqrt{-2}) & C_{4}^{*} \times C_{2}^{*} \times C_{2}^{*} & 1 & 2 & 1 \\ 4 & \mathbb{Q}(\sqrt{-(5+2 \sqrt{5})}, \sqrt{-3}, \sqrt{-1}) & C_{4}^{*} \times C_{2}^{*} \times C_{2}^{*} & 1 & 2 & 1 \\ 5 & \mathbb{Q}(\sqrt{-(5+2 \sqrt{5})}, \sqrt{-3}, \sqrt{-7}) & C_{4}^{*} \times C_{2}^{*} \times C_{2}^{*} & 1 & 2 & 1 \\ 6 & \mathbb{Q}(\sqrt{-(5+2 \sqrt{5})}, \sqrt{-3}, \sqrt{-2}) & C_{4}^{*} \times C_{2}^{*} \times C_{2}^{*} & 1 & 2 & 1 \\ 7 & \mathbb{Q}(\sqrt{-(5+2 \sqrt{5})}, \sqrt{-1}, \sqrt{-7}) & C_{4}^{*} \times C_{2}^{*} \times C_{2}^{*} & 1 & 2 & 1 \\ 8 & \mathbb{Q}(\sqrt{-(2+\sqrt{2})}, \sqrt{-3}, \sqrt{-1}) & C_{4}^{*} \times C_{2}^{*} \times C_{2}^{*} & 1 & 2 & 1 \\ 9 & \mathbb{Q}(\sqrt{-(2+\sqrt{2})}, \sqrt{-5}, \sqrt{-1}) & C_{4}^{*} \times C_{2}^{*} \times C_{2}^{*} & 1 & 1 & 1 \\ 10 & \mathbb{Q}(\theta, \sqrt{3}) & C_{8}^{*} \times C_{2} & 4 & 1 & 1\end{array}$

every normal CM-field $M_{16.1}$ with $G\left(M_{16.1} / \mathbb{Q}\right)=G_{4}$ and $M_{16.1}^{+}=F_{8}$. Since $M_{16.1} / \mathbb{Q}(\sqrt{5})$ and $M_{16.1} / \mathbb{Q}(\sqrt{13})$ are cyclic, the unique prime ideal $\mathfrak{P}_{5}$ lying above 5 in $M_{16.1}^{+}$and the unique prime ideal $\mathfrak{P}_{13}$ lying above 13 in $M_{16.1}^{+}$are ramified in $M_{16.1} / M_{16.1}^{+}$. Since if $\mathcal{F}\left(M_{16.1} / F_{8}\right) \neq \mathfrak{P}_{5} \mathfrak{P}_{13}$, then at least four prime ideals are ramified in $M_{16.1} / F_{8}$ and $8 \mid h_{M_{16.1}}^{-}$, we assume that $\mathcal{F}\left(M_{16.1} / F_{8}\right)=\mathfrak{P}_{5} \mathfrak{P}_{13}$. Then $M_{16.1}$ is equal to the field $K$ defined in Lemma 7, point (7). Let $L_{8}$ be any one of two non-normal octic CM-subfields of $M_{16.1}$. Then $L_{8}^{+}=\mathbb{Q}(\sqrt{(65+\sqrt{65}) / 2})$ or $\mathbb{Q}(\sqrt{(65+7 \sqrt{65}) / 2})$. We verify that there is no totally imaginary quadratic extension of $\mathbb{Q}(\sqrt{(65+7 \sqrt{65}) / 2})$ of conductor $\mathfrak{p}_{5} \mathfrak{p}_{13} \infty$, where $\mathfrak{p}_{5}$ (resp. $\mathfrak{p}_{13}$ ) is the prime ideal lying above 5 (resp. 13) in $\mathbb{Q}(\sqrt{(65+7 \sqrt{65}) / 2})$. Then $L_{8}^{+}=$ $\mathbb{Q}(\sqrt{(65+\sqrt{65}) / 2})$ and $\mathcal{F}\left(L_{8} / L_{8}^{+}\right)=\mathcal{I}_{5} \mathcal{I}_{13}$, where $(5)=\mathcal{I}_{5}^{4}$ and $(13)=\mathcal{I}_{13}^{4}$ in $L_{8}^{+}$. We verify that $h_{L_{8}}^{-}=10$, whence the normal closure of $L_{8}$ has relative class number $\left(h_{L_{8}}^{-}\right)^{2} / 2=50$. This proves that $h_{M_{16.1}}^{-}>2 Q_{M_{16.1}}$.

(ii) We now let $M_{16.2}=\mathbb{Q}(\sqrt{-(5+2 \sqrt{5})}, \sqrt{-(2+\sqrt{2})})$ with

$$
M_{16.2}^{+}=\mathbb{Q}(\sqrt{10+\sqrt{10}}, \sqrt{5})
$$

(see [CK] and $\left[\mathrm{G}\right.$ ). By the similar way we verify that every normal CM-field $M_{16.1}$ with $G\left(M_{16.1} / \mathbb{Q}\right)=G_{4}$ and $M_{16.1}^{+}=\mathbb{Q}(\sqrt{10+\sqrt{10}}, \sqrt{5})$ has $h_{M_{16.1}}^{-}>2 Q_{M_{16.1}}$. This completes the proof.

Proposition 26. There are exactly three composita $N=M_{16.1} M_{16.2}$ such that $G\left(M_{16.1} / \mathbb{Q}\right)=G_{4}, G\left(M_{16.2} / \mathbb{Q}\right)=C_{4}^{*} \times C_{2}^{*} \times C_{2}^{*}$, and $h_{N}^{-}=1:$

$$
\mathbb{Q}(\theta, \sqrt{2}, \sqrt{-1}), \mathbb{Q}(\theta, \sqrt{3}, \sqrt{-1}), \mathbb{Q}(\theta, \sqrt{7}, \sqrt{-1})
$$


with $\theta^{8}+10 \theta^{6}+25 \theta^{4}+20 \theta^{2}+5=0$. Moreover, these three fields have class number one.

Proof. For the seven fields $M_{16.2}$ numbered 3-9 in Table 4 of Proposition 24 we will construct normal CM-fields $M_{16.1}$ containing the given field $M_{16.2}^{+}$such that at most two prime ideals are ramified in the extension $M_{16.1} / M_{16.2}^{+}$. Let $G_{4}$ be as Proposition 9. We have $M_{16.1}^{+}=\left\langle a^{4}\right\rangle^{\prime}, G\left(M_{16.1} /\langle a\rangle^{\prime}\right)=G\left(M_{16.1} /\langle a b\rangle^{\prime}\right)=$ $C_{8}, G\left(M_{16.1} /\left\langle a^{4}, b\right\rangle^{\prime}\right)=C_{2} \times C_{2}$, and $G\left(M_{16.1} /\left\langle a^{2} b\right\rangle^{\prime}\right)=C_{4}$. The field $M_{16.1}$ has two isomorphic octic non-normal CM-subfields, $M_{8.1}=\left\langle a^{4} b\right\rangle^{\prime}$ and $M_{8.2}=\langle b\rangle^{\prime}$. Then $h_{M_{16.1}}^{-}=\frac{Q_{M_{16.1}}}{2}\left(\frac{h_{M_{8.1}}^{-}}{Q_{M_{8.1}}}\right)^{2}$. Therefore, $h_{M_{16.1}}^{-}=2 Q_{M_{16.1}}$ implies $h_{M_{8.1}}^{-}=2 Q_{M_{8.1}}$. In order to have $h_{N}^{-}=1$ there are at most two ramified primes in $M_{8.1} / M_{8.1}^{+}$.

(i) Let $M_{16.2}=\mathbb{Q}(\sqrt{-(5+2 \sqrt{5})}, \sqrt{-1}, \sqrt{-2})$. Let us construct the CM-fields $M_{16.1}$ containing $M_{16.2}^{+}=\mathbb{Q}(\sqrt{(5-\sqrt{5}) / 2}, \sqrt{2})$. Then $\left\langle a^{4}, b\right\rangle^{\prime}\left(=M_{8.1}^{+}=M_{8.2}^{+}\right)=$ $\mathbb{Q}(\sqrt{(5-\sqrt{5}) / 2})$ or $\mathbb{Q}(\sqrt{5+\sqrt{5}})$.

(i-a) Assume that $M_{8.1}^{+}=M_{8.2}^{+}=\mathbb{Q}(\sqrt{(5-\sqrt{5}) / 2})$. Then $\left\langle a^{2} b\right\rangle^{\prime}=\mathbb{Q}(\sqrt{5+\sqrt{5}})$ and $M_{16.1} / \mathbb{Q}(\sqrt{5+\sqrt{5}})$ is cyclic. In $M_{8.1}^{+}(5)=\mathfrak{p}_{5}^{4}$ and $(2)=\mathfrak{p}_{2}^{2}$. Then

$$
\mathcal{F}\left(M_{8.1} / M_{8.1}^{+}\right)=\mathfrak{p}_{2}^{e} \mathfrak{p}_{5}
$$

with $e=2,4$, or 5 , and $M_{8.1}=\mathbb{Q}(\theta)$ with $\theta^{8}+10 \theta^{6}+25 \theta^{4}+20 \theta^{2}+5=0$, by Lemma 7 , point (8). We verify that $h_{M_{8.1}}^{-}=2$, whence $h_{M_{16.1}}^{-}=2$. Hence the relative class number of the field $N=M_{8.1}(\sqrt{2}, \sqrt{-1})$ is equal to 1 . For convenience we write $\mathbb{Q}(\theta)=F_{8}$. Note that $N^{+}=L(\sqrt{2})$ and $h_{N^{+}}=1$, where $L=\mathbb{Q}(\eta)$ with $\eta^{8}-10 \eta^{6}+25 \eta^{4}-20 \eta^{2}+5=0$.

(i-b) Assume now that $M_{8.1}^{+}=M_{8.2}^{+}=\mathbb{Q}(\sqrt{5+\sqrt{5}})$. Then $M_{16.1} /$ $\mathbb{Q}(\sqrt{(5-\sqrt{5}) / 2})$ is cyclic. In $M_{8.1}^{+}(5)=\mathfrak{p}_{5}^{4}$ and $(2)=\mathfrak{p}_{2}^{2}$. Then $\mathcal{F}\left(M_{8.1} / M_{8.1}^{+}\right)=$ $\mathfrak{p}_{2}^{e} \mathfrak{p}_{5}$ with $e=2,4$, or 5 , and according to Lemma 7 , point (9), there is no such field $M_{8.1}$.

(ii) Let $M_{16.2}=\mathbb{Q}(\sqrt{-(5+2 \sqrt{5})}, \sqrt{-3}, \sqrt{-1})$. By a similar way as (i) we get $M_{16.1}=F_{8}(\sqrt{3})$ with $h_{M_{16.1}}^{-}=2$, whence $N=F_{8}(\sqrt{3}, \sqrt{-1})$ has $h_{N}^{-}=1$. We verify that $h_{N^{+}}=1$.

(iii) Let $M_{16.2}=\mathbb{Q}(\sqrt{-(5+2 \sqrt{5})}, \sqrt{-3}, \sqrt{-7})$. Let $M_{16.1}$ be a normal CMfield with $G\left(M_{16.1} / \mathbb{Q}\right)=G_{4}$ containing $M_{16.2}^{+}=\mathbb{Q}(\sqrt{3(5+\sqrt{5}) / 2}, \sqrt{21})$. Note that (5) $=\mathcal{I}_{1}{ }^{4} \mathcal{I}_{2}{ }^{4}$ in $\mathbb{Q}(\sqrt{3(5+\sqrt{5}) / 2}, \sqrt{21}), \mathcal{I}_{1}$ and $\mathcal{I}_{2}$ are ramified in $M_{16.1} / M_{16.1}^{+}$. $\left\langle a^{2} b\right\rangle^{\prime}=\mathbb{Q}(\sqrt{7(5-\sqrt{5}) / 2})$ or $\mathbb{Q}(\sqrt{3(5-\sqrt{5}) / 2})$.

(iii-a) If $M_{16.1} / \mathbb{Q}(\sqrt{7(5-\sqrt{5}) / 2})$ is cyclic, then the prime ideal lying above 3 is ramified in $M_{16.1} / \mathbb{Q}(\sqrt{3(5+\sqrt{5}) / 2}, \sqrt{21})$, whence $Q_{M_{16.1}}=1$ and $4 \mid h_{M_{16.1}}^{-}$.

(iii-b) If $M_{16.1} / \mathbb{Q}(\sqrt{3(5-\sqrt{5}) / 2})$ is cyclic, then the prime ideal lying above 7 is ramified in $M_{16.1} / \mathbb{Q}(\sqrt{3(5+\sqrt{5}) / 2}, \sqrt{21})$, whence $Q_{M_{16.1}}=1$ and $4 \mid h_{M_{16.1}}^{-}$. 
(iv) Let $M_{16.2}=\mathbb{Q}(\sqrt{-(5+2 \sqrt{5})}, \sqrt{-3}, \sqrt{-2})$. By the same argument as (iii) we prove that every normal CM-field $M_{16.1}$ with $G\left(M_{16.1} / \mathbb{Q}\right)=G_{4}$ containing $M_{16.2}^{+}=\mathbb{Q}(\sqrt{3(5+\sqrt{5}) / 2}, \sqrt{6})$ satisfies $4 \mid h_{M_{16.1}}^{-}$.

(v) Let $M_{16.2}=\mathbb{Q}(\sqrt{-(5+2 \sqrt{5})}, \sqrt{-1}, \sqrt{-7})$. By a similar way as (i) we obtain $M_{16.1}=F_{8}(\sqrt{7})$ with $h_{M_{16.1}}^{-}=2$, whence $N=F_{8}(\sqrt{7}, \sqrt{-1})$ has $h_{N}^{-}=1$. We verify that $h_{N^{+}}=1$.

(vi) Let $M_{16.2}=\mathbb{Q}(\sqrt{-(2+\sqrt{2})}, \sqrt{-3}, \sqrt{-1})$ with $M_{16.2}^{+}=\mathbb{Q}(\sqrt{2+\sqrt{2}}, \sqrt{3})$. Then $\left\langle a^{2} b\right\rangle^{\prime}=\mathbb{Q}(\sqrt{3(2+\sqrt{2})})$ or $\mathbb{Q}(\sqrt{2+\sqrt{2}})$.

(vi-a) Assume that $M_{16.1} / \mathbb{Q}(\sqrt{3(2+\sqrt{2})})$ is cyclic. In $\mathbb{Q}(\sqrt{2+\sqrt{2}})(2)=\mathfrak{p}_{2}^{4}$ and (3) remains prime. However, according to Lemma 7, point (10), the octic subfield $M_{8.1}$ of $M_{16.1}$ cannot satisfy $h_{M_{8.1}}^{-}=2 Q_{M_{8.1}}$.

(vi-b) Assume now that $M_{16.1} / \mathbb{Q}(\sqrt{2+\sqrt{2}})$ is cyclic. Then the prime ideal lying above 3 is totally ramified in $M_{16.1} / \mathbb{Q}(\sqrt{2+\sqrt{2}})$. In $\mathbb{Q}(\sqrt{3(2+\sqrt{2})})(2)=\mathfrak{p}_{2}^{4}$ and (3) $=\mathfrak{p}_{3}^{2}$. The field $K$ defined in Lemma 7 , point (11), is the unique (up to isomorphism) octic CM-field with $K^{+}=\mathbb{Q}(\sqrt{3(2+\sqrt{2})})$ such that at most two prime ideals are ramified in $K / K^{+}$and the normal closure $N$ of $K$ has $G(N / \mathbb{Q})=$ $G_{4}$ and $N^{+}=\mathbb{Q}(\sqrt{2+\sqrt{2}}, \sqrt{3})$. Set $M_{8.1}=K$. We verify that $h_{M_{8.1}}^{-}=10$.

(vii) Let $M_{16.2}=\mathbb{Q}(\sqrt{-(2+\sqrt{2})}, \sqrt{-5}, \sqrt{-1})$. By a similar computation as (vi) we verify that there is no normal CM-field $M_{16.1}$ containing

$$
M_{16.2}^{+}=\mathbb{Q}(\sqrt{2+\sqrt{2}}, \sqrt{5})
$$

such that $G\left(M_{16.1} / \mathbb{Q}\right)=G_{4}$ and $h_{M_{16.1}}^{-} \mid 4$. The proof is completed.

Proposition 27. (1) If $G\left(M_{16.1} / \mathbb{Q}\right)=G\left(M_{16.2} / \mathbb{Q}\right)=G_{5}$, then $h_{N}^{-} \geq 2$.

(2) If $G\left(M_{16.1} / \mathbb{Q}\right)=G_{5}$ and $G\left(M_{16.2} / \mathbb{Q}\right)=G_{6}$, then $h_{N}^{-} \geq 2$.

(3) If $G\left(M_{16.1} / \mathbb{Q}\right)=G_{5}$ and $G\left(M_{16.2} / \mathbb{Q}\right)=C_{4}^{*} \times C_{4}^{*}$, then $h_{N}^{-} \geq 2$.

(4) If $G\left(M_{16.1} / \mathbb{Q}\right)=G_{5}$ and $G\left(M_{16.2} / \mathbb{Q}\right)=C_{4}^{*} \times C_{2}^{*} \times C_{2}^{*}$, then there is one and only one $N$ with $N=M_{16.1} M_{16.2}$ and $h_{N}^{-}=1$ :

$$
N=\mathbb{Q}(\sqrt{2+\sqrt{2}}, \sqrt{3+\sqrt{3}}, \sqrt{-1}) .
$$

(5) If $G\left(M_{16.1} / \mathbb{Q}\right)=G_{5}$ and $G\left(M_{16.2} / \mathbb{Q}\right)=C_{8}^{*} \times C_{2}$, then $h_{N}^{-}>1$.

Proof. Let $M_{8.1}, M_{8.2}, L_{8.1}$, and $L_{8.2}$ be four normal CM-fields such that $M_{16.1}=$ $M_{8.1} M_{8.2}, M_{16.2}=L_{8.1} L_{8.2}, M_{8.1}^{+}=M_{8.2}^{+}=L_{8.1}^{+}=L_{8.2}^{+}$.

(1) We have $G\left(M_{8.1} / \mathbb{Q}\right)=G\left(L_{8.1} / \mathbb{Q}\right)=D_{8}$ and $G\left(M_{8.2} / \mathbb{Q}\right)=G\left(L_{8.2} / \mathbb{Q}\right)=$ $Q_{8}$. Then the compositum $M_{8.2} L_{8.2}$ is a normal CM-field with Galois group $G_{8}$. According to Proposition 19 (3) $h_{M_{8.2} L_{8.2}}^{-} \geq 8$, hence $h_{N}^{-} \geq 2$.

(2) Let $M_{8.1}^{+}=M_{8.2}^{+}=L_{8.1}^{+}=L_{8.2}^{+}=\mathbb{Q}\left(\sqrt{m_{1}}, \sqrt{m_{2}}\right)$. We have $G\left(M_{8.1} / \mathbb{Q}\right)=$ $G\left(L_{8.1} / \mathbb{Q}\right)=G\left(L_{8.2} / \mathbb{Q}\right)=D_{8}$ and $G\left(M_{8.2} / \mathbb{Q}\right)=Q_{8}$. If $h_{N}^{-}=1$, then $h_{M_{8.1}}^{-}, h_{L_{8.1}}^{-}$, and $h_{L_{8.2}}^{-}$divide 4 and $\left(m_{1}, m_{2}\right) \in\{(2,17),(5,29)\}$ by [LO1] and [YK]. If $\left(m_{1}, m_{2}\right)$ $=(2,17)$, then two prime ideals lying above 2 and two prime ideals lying above 17 
are ramified in $M_{8.2}$. Hence $8 \mid h_{M_{8.2}}^{-}$. By the same argument if $\left(m_{1}, m_{2}\right)=(5,29)$, then $8 \mid h_{M_{8,2}}^{-}$. It follows that $h_{N}^{-} \geq 2$ (cf. Lemma 9).

(3) This case is treated in Proposition 16 (3). (See Remark 2 (b).)

(4) This case is treated in Proposition 16 (4). (See Remark 2 (c).)

(5) Suppose that $h_{N}^{-}=1$. Then $h_{M_{16.2}}^{-} \mid 4$, whence $M_{16.2}$ must be the field numbered 10 in Table 4 . The unique real biquadratic bicyclic subfield of $M_{16.2}^{+}$is $\mathbb{Q}(\sqrt{3}, \sqrt{17})$. By $[\mathrm{K}, 2.2$ Proposition] $\mathbb{Q}(\sqrt{3}, \sqrt{17})$ cannot be embedded in an octic quaternion field. Hence, there is no field $M_{16.1}$ containing $\mathbb{Q}(\sqrt{3}, \sqrt{17})$, and we conclude that $h_{N}^{-}>1$.

Proposition 28. (1) If $G\left(M_{16.1} / \mathbb{Q}\right)=G\left(M_{16.2} / \mathbb{Q}\right)=G_{6}$, then $h_{N}^{-}>1$.

(2) If $G\left(M_{16.1} / \mathbb{Q}\right)=G_{6}$ and $G\left(M_{16.2} / \mathbb{Q}\right)$ is abelian, then $h_{N}^{-}>1$.

Proof. (1) It is sufficient to notice that there is no quadruple of octic dihedral CM-fields with the same maximal real subfield whose relative class numbers divide 4.

(2) If $G\left(M_{16.2} / \mathbb{Q}\right)=C_{4}^{*} \times C_{4}^{*}$ or $C_{4}^{*} \times C_{2}^{*} \times C_{2}$, then $h_{N}^{-} \geq 2$ (see Remark 4). Suppose that $G\left(M_{16.2} / \mathbb{Q}\right)=C_{8}^{*} \times C_{2}$ and $h_{N}^{-}=1$. From Remark 3 (a) it follows that the unique biquadratic bicyclic real subfield $\mathbb{Q}\left(\sqrt{m_{1}}, \sqrt{m_{2}}\right)$ of $M_{16.1}^{+}=M_{16.2}^{+}$ satisfies $\left(m_{1}, m_{2}\right) \in S$ where $S$ is as in Remark 3. The fact that $h_{M_{16.2}}^{-} \mid 4$ implies $\left(m_{1}, m_{2}\right)=(51,3)$. Since $(51,3) \notin S$, we have a contradiction.

In conclusion the proof of point (2) of Theorem 1 is completed by Propositions $11-28$.

\section{ACKNowledgements}

All computations were performed using KASH ([KT) and Pari-GP $([\mathrm{P}]$ ). S.-H. Kwon is greatly indebted to C. Fieker for helps concerning KASH. The authors wish to express their gratitude to S. Louboutin for several helpful suggestions during the preparation of this version. S.-H. Kwon was supported by the Korea Research Foundation under grant KRF-2005-C00002.

\section{REFERENCES}

[BLS] E. Benjamin, F. Lemmermeyer, and C. Snyder, Real quadratic fields with abelian 2-class field tower, J. Number Theory, 73 (1998), 182-194. MR.1658015 (2000c:11179)

[CK] K.-Y.Chang and S.-H. Kwon, Class numbers of imaginary abelian number fields, Proc. Amer. Math. Soc., Vol. 128, No. 9 (2000), 2517-2528. MR.1707511 (2000m:11108)

[Co] H.Cohen, Advanced topics in computational number theory, Springer-Verlag Graduate Texts in Math. 193, 2000. MR1728313 (2000k:11144)

[G] M.-N. Gras, Classes et unites des extensions cycliques reelles de degre 4 de $\mathbb{Q}$, Ann. Inst. Fourier, 29 no 1, xiv (1979), 102-124. MR.526779 (81f:12003)

$[\mathrm{H}] \quad$ H. Hasse, Uber die Klassenzahl abelscher Zahlkorper, Akademie-Verlag : Berlin, 1952. Reprinted with an introduction by J. Martinet : Springer-Verlag : Berlin, 1985. MR842666 (87j:11122a)

[He] E. Hecke, Lectures on the theory of algebraic numbers, Springer-Verlag Graduate Texts in Math. 77, 1981. MR638719 (83m:12001)

[JL] G. James and M. Liebeck, Representations and characters of groups, Cambridge University Press, 1993. MR1237401 (94h:20007)

[K] S.-H. Kwon, Sur les discriminants minimaux des corps quaternioniens, Arch. Math., 67 (1996), 119-125. MR.1399827(97d:11165)

[KT] M. Daberkow, F. Fieker, J. Kluners, M. Pohst, K. Roegner and K. Wildanger, KANT $V_{4}$, J. Symbolic Computation, 24 (1997), 267-283. MR1484479 (99g:11150) 
[Lef] Y. Lefeuvre, Corps diédraux à multiplication complexe principaux, Ann. Inst. Fourier, 50.1 (2000), 67-103. MR.1762338 (2001g:11166)

[Lem1] F. Lemmermeyer, Ideal class groups of cyclotomic number fields I, Acta Arith., 72.4 (1995), 347-359. MR1348202(96h:11111)

[Lem2] F. Lemmermeyer, Unramified quaternion extensions of quadratic number fields, J. de Théorie des Nombres de Bordeaux, 9 (1997), 51-68. MR 1469661 (98j:11090)

[LO1] S. Louboutin and R. Okazaki, Determination of all non-normal quartic CM-fields and of all non-abelian normal octic CM-fields with class number one, Acta Arith., 67 (1994), 47-62. MR 1292520 (95g:11107)

[LO2] S. Louboutin and R. Okazaki, The class number one problem for some non-abelian normal CM-fields of 2-power degree, Proc. London Math. Soc., 76 part 3 (1998), 523-548. MR 1616805 (99c:11138)

[LOO] S. Louboutin, R. Okazaki, and M. Olivier, The class number one problem for some non-abelian normal CM-fields, Trans. of the A.M.S., Vol.349, No.9 (1997), 3657-3678. MR.1390044 (97k:11149)

[Lou1] S. Louboutin, Lower bounds for relative class numbers of CM-fields, Proc. Amer. Math. Soc., Vol 120 (1994), 425-434. MR1169041 (94d:11089)

[Lou2] S. Louboutin, Determination of all quaternion octic CM-fields with class number 2, J. London Math. Soc., (2) 54 (1996), 227-238. MR.1405052 (97g:11122)

[Lou3] S. Louboutin, The class number one problem for the non-abelian normal CM-fields of degree 16, Acta Arith., 82.2 (1997), 173-196. MR1477509 (98j:11097)

[Lou4] S. Louboutin, Majoration explicites du résidu au point 1 des fonctions zêta de certains corps de nombres, J. Math. Soc. Japan, Vol. 50 (1998), 57-69. MR.1484611 (99a:11131)

[Lou5] S. Louboutin, Upper bounds on $|L(1, \chi)|$ and applications, Canada J. Math. Vol. 50(4) (1998), 794-815. MR1638619 (99f:11149)

[Lou6] S. Louboutin, The class number one problem for the dihedral and dicyclic CM-fields, Colloquium Mathematicum, Vol. 80, No. 2 (1999), 259-265. MR.1703822 (2000e:11140)

[Lou7] S. Louboutin, Computation of relative class numbers of CM-fields by using Hecke Lfunctions, Math. Comp., Vol.69 (2000), 371-393. MR.1648395 (2000i:11172)

[Lou8] S. Louboutin, Computation of $L(0, \chi)$ and of relative class numbers of CM-fields, Nagoya Math. J., Vol 161 (2001), 171-191. MR.1820217(2002e:11152)

[Lou9] S. Louboutin, Explicit upper bounds for residues of Dedekind zeta functions and values of $L$-functions at $s=1$, and explicit lower bounds for relative class numbers of CM-fields, Can. J. Math. 53 (2001), 1194-1222. MR1863848 (2003d:11167)

[Lou10] S. Louboutin, Explicit lower bounds for residues at $s=1$ of Dedekind zeta functions and relative class numbers of CM-fields, Trans. Amer. Math. Soc., 355 (2003), 3079-3098. MR:1974676 (2004f:11134)

[Mar] J. Martinet, Une introduction à la théorie du corps de classes (notes de M. Olivier), Ecole dotorale de Mathématiques de Bordeaux (1991).

[Mas] J. M. Masley, Class numbers of real cyclic number fields with small conductor, Compositio Mathematica, Vol. 37. Fasc. 3 (1978), 297-319. MR.511747 (80e:12005)

[N] J. Neukirch, Class field theory, A Series of Comprehensive studies in Mathematics 280, Springer-Verlag (1986). MR.819231 (87i:11005)

[Ok] R. Okazaki, Inclusion of CM-fields and divisibility of relative class numbers, Acta Arith., 112.4 (2000), 319-338. MR1760241 (2001h:11138)

[P] C. Batut, K. Belabas, D. Bernardi, H. Cohen, M. Olivier, PARI-GP version 2.0.11.

[PK] Y.-H. Park and S.-H. Kwon, Determination of all non-quadratic imaginary cyclic number fields of 2-power degree with relative class number $\leq 20$, Acta Arith., 83.3 (1998), 211223. MR 1611205 (99a:11125)

[R1] O. Ramaré, Approximate formulae for $L(1, \chi)$, Acta, Arith., C.3 (2001), 245-266. MR.1865385 (2002k:11144)

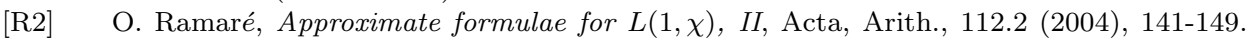
MR2051374(2005g:11156)

[S] J.-P. Serre, Corps lacaux, Hermann, $3^{e}$ édition, 1980.

[TW] A.D. Thomas and G.V. Wood, Group Tables, Shiva Mathematics series 2, 1980. MR572793 (81d:20002) 
[W] L.C. Washington, Introduction to Cyclotomic Fields, Springer-Verlag, Grad. Texts Math. Second Edition, 1997. MR1421575 (97h:11130)

[YK] H.-S. Yang and S.-H. Kwon, The non-normal quartic CM-fields and the octic dihedral CM-fields with relative class number two, J. Number Theory, 79 (1999), 175-193. MR.1728146 (2000h:11117)

Department of Mathematics, Korea University, 136-701, Seoul, Korea

E-mail address: smpark@korea.ac.kr

Department of Mathematics, Korea University, 136-701, Seoul, Korea

Current address: Korea Minting and Security Printing Corporation, 54, Gwahakro, YusongGu, 305-713 Daejon, Korea

E-mail address: yanghs@komsco.com

Department of Mathematics Education, Korea University, 136-701, Seoul, Korea

E-mail address: sounhikwon@korea.ac.kr 